\title{
AN AGENT-BASED MODEL FOR SECONDARY USE OF RADIO SPECTRUM
}

\author{
by \\ Arnon Tonmukayakul \\ B.E. in Computer Engineering, Chulalongkorn University, 2000 \\ M.S. in Telecommunications, University of Pittsburgh, 2001 \\ Submitted to the Graduate Faculty of \\ the School of Information Sciences in partial fulfillment \\ of the requirements for the degree of \\ Doctor of Philosophy
}

University of Pittsburgh

2007 


\section{UNIVERSITY OF PITTSBURGH \\ SCHOOL OF INFORMATION SCIENCES}

This dissertation was presented

by

Arnon Tonmukayakul

It was approved by

Martin B. H. Weiss, PhD, Associate Professor, School of Information Sciences Richard A. Thompson, PhD, Professor, School of Information Sciences Prashant Krishnamurthy, PhD, Associate Professor, School of Information Sciences Michael Lewis, PhD, Professor, School of Information Sciences John Duffy, PhD, Professor, Department of Economics

Dissertation Director: Martin B. H. Weiss, PhD, Associate Professor, School of Information Sciences 
Copyright (c) by Arnon Tonmukayakul

2007 


\title{
AN AGENT-BASED MODEL FOR SECONDARY USE OF RADIO SPECTRUM
}

\author{
Arnon Tonmukayakul, PhD \\ University of Pittsburgh, 2007
}

Wireless communications rely on access to radio spectrum. With a continuing proliferation of wireless applications and services, the spectrum resource becomes scarce. The measurement studies of spectrum usage, however, reveal that spectrum is being used sporadically in many geographical areas and times. In an attempt to promote efficiency of spectrum usage, the Federal Communications Commission has supported the use of market mechanism to allocate and assign radio spectrum. We focus on the secondary use of spectrum defined as a temporary access of existing licensed spectrum by a user who does not own a spectrum license. The secondary use of spectrum raises numerous technical, institutional, economic, and strategic issues that merit investigation. Central to the issues are the effects of transaction costs associated with the use of market mechanism and the uncertainties due to potential interference.

The research objective is to identify the pre-conditions as to when and why the secondary use would emerge and in what form. We use transaction cost economics as the theoretical framework in this study. We propose a novel use of agent-based computational economics to model the development of the secondary use of spectrum. The agent-based model allows an integration of economic and technical considerations to the study of pre-conditions to the secondary use concept. The agent-based approach aims to observe the aggregate outcomes as a result of interactions among agents and understand the process that leads to the secondary use, which can then be used to create policy instruments in order to obtain the favorable outcomes of the spectrum management. 


\section{TABLE OF CONTENTS}

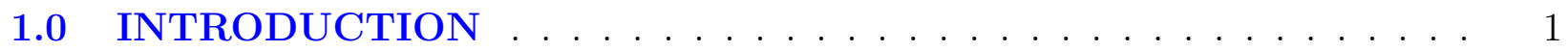

1.1 Background . . . . . . . . . . . . . . . . . . . . 1

1.1.1 Radiocommunications Spectrum . . . . . . . . . . . . 1

1.1.2 Spectrum Scarcity and Harmful Interference . . . . . . . . . . 2

1.2 Motivation . . . . . . . . . . . . . . . . . . 6

1.3 Problem Statement . . . . . . . . . . . . . . . . . . . . . . 7

1.4 Dissertation Outline . . . . . . . . . . . . . . . . . 7

2.0 SPECTRUM SHARING TECHNIQUES $\ldots \ldots \ldots \ldots \ldots$

2.1 Spectrum Dimensioning . . . . . . . . . . . . . . . . 9

2.2 Taxonomy of Spectrum Sharing . . . . . . . . . . . . . . . . 12

2.3 Cooperative Sharing Techniques . . . . . . . . . . . . . . . . . 14

2.3.1 Spectrum Trading and Leasing . . . . . . . . . . . . . . 14

2.3.2 Interruptible Spectrum Sharing . . . . . . . . . . . . 18

2.3 .3 Spectrum Pooling . . . . . . . . . . . . . . . . . 19

2.3 .4 Virtual Network Operators . . . . . . . . . . . . . . . 20

2.4 Coexistent Sharing Techniques . . . . . . . . . . . . . . . . . . 21

2.4.1 Unlicensed Spectrum Access _. . . . . . . . . . . . . . . 21

2.4 .2 Opportunistic Spectrum Access . . . . . . . . . . . . . . 23

2.5 Summary . . . . . . . . . . . . . . . . . . . . . . . . . . 24

3.0 THEORETICAL FRAMEWORK AND COMPUTATIONAL TOOL 26

3.1 Transaction Cost Economics (TCE) . . . . . . . . . . . . . 26

3.2 Agent-Based Computational Economics (ACE) . . . . . . . . . . 30 
4.0 RESEARCH DESIGN AND RESEARCH QUESTIONS . . . . . . . 33

4.1 Research Outline . . . . . . . . . . . . . . . . . . . . 33

4.2 Research Questions . . . . . . . . . . . . . . . . . . . . 36

4.3 Experimental Design . . . . . . . . . . . . . . . . . . . 37

5.0 ISSUES IN SECONDARY USE OF RADIO SPECTRUM . . . . . . . 41

5.1 Why would a primary user wants to share portions of his/her spectrum for

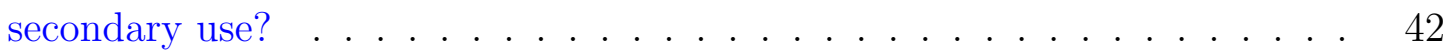

5.2 Why would a spectrum user wants to become a secondary user? . . . . . . 43

5.3 How do primary and secondary users find each other? . . . . . . . . . . 44

5.4 What has to be negotiated? and how? . . . . . . . . . . . . 45

6.0 AN AGENT-BASED MODEL FOR SECONDARY USE OF RADIO SPECTRUM . . . . . . . . . . . . . . . . . . . 46

6.1 Model Overview . . . . . . . . . . . . . . . . . . . . . . . 46

6.2 Transaction Cost Economics and Secondary Use of Spectrum . . . . . . . 47

6.3 Agents . . . . . . . . . . . . . . . . . . . . 49

6.3 .1 Spectrum Users . . . . . . . . . . . . . . . . . . . 50

6.3 .2 Consumer Agents . . . . . . . . . . . . . . . . . . 51

6.3 .3 Provider Agents . . . . . . . . . . . . . . . . . 56

6.4 Learning Algorithm . . . . . . . . . . . . . . . . . . . . . . 59

6.4 .1 Consumer Learning . . . . . . . . . . . . . . . . . . 59

6.4 .2 Provider Learning . . . . . . . . . . . . . . . . . . . 62

6.5 Opportunistic Behavior Modeling . . . . . . . . . . . . . 65

6.5.1 Consumer Opportunistic Behavior _ . . . . . . . . . . 65

6.5 .2 Provider Opportunistic Behavior . . . . . . . . . . . . . 69

6.6 Spectrum Leasing and Pricing . . . . . . . . . . . . . . . . 71

6.7 Operating Environment . . . . . . . . . . . . 76

6.8 Stopping Criteria . . . . . . . . . . . . . . . . . 77

$7.0 \quad$ RESULTS AND DISCUSSION . . . . . . . . . . . . . . . . . 79

7.1 Model Setup . . . . . . . . . . . . . . . . . . . . . . . . . . . 79

7.2 Model Validation and The Existence of Secondary Use . . . . . . . . 81 
7.2.1 Spectrum Access Without Secondary Use . . . . . . . . . . . 83

7.2.2 Spectrum Access With Secondary Use . . . . . . . . . . . 86

7.2 .3 Secondary Use with Opportunism $\ldots \ldots \ldots \ldots$

7.3 Q1: Number of Participants . . . . . . . . . . . . . . . . . 90

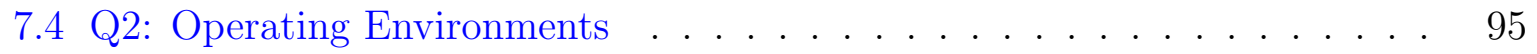

7.5 Q3: Spectrum Usage Characteristics of Primary Users . . . . . . . . . 100

7.6 Q4: Coverage Requirements . . . . . . . . . . . . . . . 106

7.7 Q5: Application Requirements . . . . . . . . . . . . . . . . . . 112

7.8 Q6: Expansion of Unlicensed Spectrum . . . . . . . . . . . . . 117

7.9 Implications and Limitations of Results . . . . . . . . . . . . . . 123

8.0 CONCLUSION AND FUTURE WORK . . . . . . . . . . . . 125

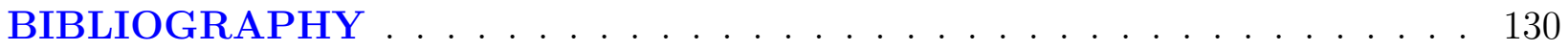




\section{LIST OF TABLES}

$1.13 \mathrm{G}$ Spectrum Auction Results of Germany and the U.K. . . . . . . . . . . 5

1.2 Top 10 Bidders in Spectrum Auction of Advanced Wireless Services in the U.S. 5

2.1 Dimensions of Radio Spectrum Space . . . . . . . . . . . . . . . . . 11

2.2 Spectrum Access Approaches of Primary Users . . . . . . . . . . . . . . 13

2.3 Spectrum Access Approaches of Secondary Users . . . . . . . . . . . . 13

$4.1 \quad$ Full Factorial Design for Q1 . . . . . . . . . . . . . . . . . . 38

4.2 Full Factorial Design for Q2 and Q3 . . . . . . . . . . . . . . 39

4.3 Full Factorial Design for Q4 and Q5 . . . . . . . . . . . . 39

4.4 Full Factorial Design for Q6 . . . . . . . . . . . . . . . . . . 40

4.5 Statistical Testing Methods . . . . . . . . . . . . . . . . . . 40

6.1 General Parameters of Spectrum Users . . . . . . . . . . . . . . 53

6.2 Additional Parameters of Consumer Agents . . . . . . . . . . . . . . 56

6.3 Additional Parameters of Provider Agents . . . . . . . . . . . . . . . . . 58

6.4 Learning Parameters of Consumer Agents . . . . . . . . . . . . . . . . . 61

6.5 Learning Parameters of Provider Agents . . . . . . . . . . . . . . . . . 64

6.6 Parameters of Consumer's Opportunistic Behavior . . . . . . . . . . . . 66

6.7 Parameters of Provider's Opportunistic Behavior . . . . . . . . . . . . 69

6.8 Parameters of the Operating Environment . . . . . . . . . . . . 77

7.1 Model Parameters . . . . . . . . . . . . . . . . . . 82

$7.2 \quad$ Full Factorial Design for Secondary Use Experiments . . . . . . . . . . . . 83

7.3 Parameters for Research Question Q1 . . . . . . . . . . . . . . 90 
7.4 Regression Analysis of Percentage of Secondary Use on Number of Consumers and Providers (Q1) . . . . . . . . . . . . . . . . . . . 94

7.5 Parameters for Research Questions Q2 and Q3 . . . . . . . . . 96

7.6 Regression Analysis of Percentage of Secondary Use on Operating Environment Parameters $(\mathrm{Q} 2) \ldots \ldots \ldots 9$

7.7 Regression Analysis of Percentage of Secondary Use on Spectrum Usage Characteristics of Primary Users (Q3) . . . . . . . . . . . . . . . 105

7.8 Parameters for Research Questions Q4 and Q5 . . . . . . . . . 107

7.9 Regression Analysis of Degree of Control on Characteristics of Secondary

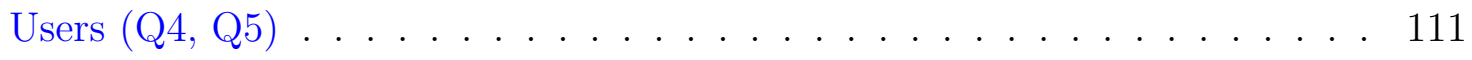

7.10 Parameters for Research Question Q6 . . . . . . . . . . . . 117

7.11 Regression Analysis of Percentage Unlicensed Use on Amount of Unlicensed Spectrum and Characteristics of Secondary Users (Q6) . . . . . . . . . 121 


\section{LIST OF FIGURES}

2.1 Traditional interference management . . . . . . . . . . . . . . . . 10

2.2 Evolution of spectrum sharing approaches. . . . . . . . . . . . . . . . 24

3.1 Governance structures in transaction cost economics. . . . . . . . . . . . . 28

6.1 Governance structures in secondary use of radio spectrum. . . . . . . . . . . 49

6.2 Utility functions: (a) inelastic application and (b) elastic application. . . . . 51

6.3 Spectrum user's wireless system: (a) one-way broadcast network, (b) two-way infrastructure network, and (c) ad hoc network. . . . . . . . . . . . 52

6.4 Degree of control values of different organizational forms in secondary use of radio spectrum. . . . . . . . . . . . . . . . . . . . . 54

6.5 Reinforcement comparison learning process. . . . . . . . . . . . . . 60

6.6 Learning process of one consumer agent. . . . . . . . . . . . . . . 63

6.7 Average surplus of ten consumer agents. . . . . . . . . . . . . . . 63

6.8 Trust development . . . . . . . . . . . . . . . . . . . . 68

6.9 Trade Price . . . . . . . . . . . . . . . . . . . . . . 76

7.1 Example of three consumer agents . . . . . . . . . . . . . . . 80

7.2 Utility function of spectrum users . . . . . . . . . . . . . . . 81

7.3 Percentage of spectrum access options $(\mathrm{NC}=5) \ldots \ldots \ldots 4$

7.4 Percentage of spectrum access options $(\mathrm{NC}=13) \ldots \ldots \ldots$

7.5 Percentage of spectrum access options $(\mathrm{NC}=19) \ldots \ldots \ldots$

7.6 Behavior of the opportunistic provider . . . . . . . . . . . . . . . . 89

7.7 Percentage of secondary use vs. Number of consumer and provider agents . 91

7.8 Contour lines of the percentage of secondary use $\ldots \ldots \ldots 1$ 
7.9 Percentage of unlicensed use vs. Number of consumer and provider agents . 92

7.10 Percentage of exclusive license use vs. Number of consumer and provider agents 93

7.11 Percentage of secondary use vs. Primary user's device density . . . . . . . . 97

7.12 Percentage of spectrum access options vs. Path-loss exponent . . . . . . 98

7.13 Percentage of secondary use vs. Spectrum use of primary user . . . . . . . . 102

7.14 Percentage of unlicensed use vs. Spectrum use of primary user . . . . . . 103

7.15 Percentage of exclusive license use vs. Spectrum use of primary user . . . 104

7.16 Average degree of control vs. Coverage area for rigid application . . . . . 108

7.17 Average degree of control vs. Coverage area for flexible application . . . . . 108

7.18 Percentage of spectrum access options vs. Coverage area for rigid application 109

7.19 Utility functions for $\mathrm{H} 5$ experiments . . . . . . . . . . . . . . . 113

7.20 Average degree of control: A comparison between rigid and flexible application requirements . . . . . . . . . . . . . . . . . . 114

7.21 Percentage of spectrum access options vs. $\alpha_{u}$ for rigid application . . . . . . 115

7.22 Percentage of unlicensed use vs. Amount of unlicensed spectrum for rigid

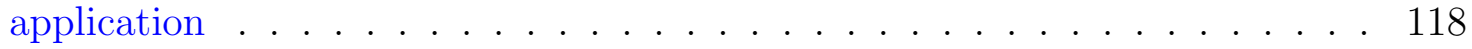

7.23 Percentage of unlicensed use: A comparison between rigid and flexible application requirements . . . . . . . . . . . . . . . . . . . . 119

7.24 Percentage of spectrum access options vs. Amount of unlicensed spectrum for rigid application requirement . . . . . . . . . . . . . . 120 


\subsection{INTRODUCTION}

Innovative wireless communications services are increasingly among the most cutting edge technologies in the past decade. During this period, wireless communications industry has grown by orders of magnitude due to enabling technologies which allow widespread deployment and smaller, more reliable, and more affordable equipments. This explosive growth has driven regulatory bodies and researchers to look into the use of radiocommunications spectrum which is the fundamental resource needed for wireless systems to function. This chapter provides a brief background of radiocommunications spectrum, current issues in spectrum management, and motivation that leads to this dissertation.

\subsection{BACKGROUND}

\subsubsection{Radiocommunications Spectrum}

In order for wireless communications to function, they must have access to a portion of radiocommunications spectrum resource. Radiocommunications spectrum (hereafter radio spectrum or spectrum) refers to the range of frequencies of electromagnetic radiation that is useful for the purposes of communications. Radio spectrum is typically defined as frequencies that lies between $9 \mathrm{kHz}$ and 3,000 GHz. Most of the current technologies, however, can practically exploit spectrum below $100 \mathrm{GHz}$. Therefore, allocation of spectrum is only identified up to $300 \mathrm{GHz}$.

Different frequencies have different properties and are suitable for different uses. In gen-

eral, lower frequencies can travel further in distance and can penetrate dense materials such 
as walls, floors, and ceilings, making them suitable for transmissions that demand large coverage areas such as radio and television broadcast. Higher frequencies can travel shorter in distance, thus making them suitable for short-range or point-to-point transmissions. These frequencies, however, can be reused to increase overall capacity in a given area. The properties of radio spectrum cause frequencies below $3 \mathrm{GHz}$ much more crowded with incumbents compared to the higher frequencies. Other technical limitations also affect the usefulness of spectrum at different frequencies. For example, certain scientific applications such as radio astronomy and meteorology need to operate in certain frequencies range and these cannot be substituted.

Radio spectrum is considered as an intangible commodity necessary to the successful deployment of wireless communications technologies and services. Its importance as a critical component of telecommunications systems is rising because of the following factors. First, spectrum access demand continues to increase due to the well-known advantages of wireless networks over wired networks in many situations, which include mobility, installation speed and flexibility, and reduced cost of ownership. Second, technological innovations of wireless services and applications such as third-generation wireless networks $(3 \mathrm{G})$, broadband wireless networks, and ad hoc networking, create a higher spectrum access demand for certain parts of radio spectrum. Third, uninterrupted access to the spectrum resource is essential for existing uses of radio spectrum to continue their operations, including military communications, public safety uses, and aeronautical communications.

Accordingly, regulators need to provide adequate access for both the existing uses and new technologies in order to create a balance between protecting the operations of existing users and fostering new wireless technologies. With a limited amount of spectrum resource, the stakes are quite high for service providers who are relying on spectrum access to maintain their operations and for those who seek to provide new services.

\subsubsection{Spectrum Scarcity and Harmful Interference}

As mentioned earlier, the amount of spectrum that is technically suitable for a particular application is limited. Spectrum scarcity is further increased because it cannot be shared 
indiscriminately. This leads to the concept of interference in technical terms which has a direct relationship to spectrum scarcity in economic terms. Wireless communications may suffer from interference when the receivers are unable to discriminate signal(s) that they are supposed to receive from other unwanted signals. Such interference is typically occurs when users are using similar frequencies at the same point of time and are within close geographical proximity to each other.

Consequently, one of the main goals of spectrum management is to avoid harmful interference among communication systems. Harmful interference is defined as interference that endangers the functioning or seriously degrades, obstructs, or repeatedly interrupts a communications system [1]. A certain amount of interference from one system, however, is allowed into another system as long as it is not considered harmful. This is the basis of interference management to define an exclusive frequency license.

In the traditional approach to managing spectrum access, which is typically referred to as a command and control regime, the government controls the spectrum and decides how frequencies are distributed. The use of spectrum in the United States is managed using a dual organization structure. The National Telecommunications and Information Administration (NTIA) manages the spectrum use of the Federal Government while the Federal Communications Commission (FCC) regulates all other uses (i.e., non-federal users). Key terms in spectrum management are allocation, use, and assignment. An allocation of spectrum designates what radiocommunications service can be used in specific frequencies. Radio services include fixed, mobile, fixed satellite, mobile satellite, broadcasting, amateur, aeronautical mobile, maritime mobile, and so forth. The NTIA and the FCC maintain the U.S. frequency allocation chart.

The regulators also determine the use of spectrum that have been allocated to a specified radio service. For example, a frequency band allocated for mobile service can be authorized to operate different uses such as cellular telephony or public safety. Uses for a frequency band allocated for broadcasting can be television broadcast or radio broadcast. Technical rules and operation guidelines are tailored to the characteristics of applications and technologies that are associated with the use of each frequency band.

An assignment, also known as an (exclusive) license or an authorization, is a process of 
granting permission to utilize a portion of spectrum resource to a specific spectrum user. Once the license has been granted, other spectrum users are not allowed to operate in those frequencies in the specified geographical area for the duration of the license. An exclusive license can be granted in a number of ways. Assignment methods include first-come, firstserved basis, comparative hearings (beauty contests), lotteries, and auctions. The auction method, however, is gaining popularity in many countries due to its nonsubjective nature and the attendant financial gains for the government.

This administrative approach in spectrum management is considered inflexible in responding to changes in technology and changes in market demand for spectrum-based services that may affect to demand for spectrum. As the spectrum management is closely tied to existing services and technologies, changes in spectrum allocation, use, and assignment may take several years and involve complex negotiation processes and lobbying activities both domestically and internationally. Accordingly, critics often argue that spectrum scarcity is artificial because of the command and control regime. More specifically, this traditional approach is mainly focused on minimizing interference rather than maximizing economic benefits of using spectrum resource. Since incumbent license holders are protected from interference, they have little or no incentive to improve the efficiency of their spectrum use. As a result, incumbent users do not operate efficiently as they could, leading to spectrum scarcity.

In the United States and many countries, the process of assigning licenses to commercial uses has settled upon the use of auctions. Tables 1.1 and 1.2 show industries' willingness-topay for spectrum through the results of the spectrum auctions for $3 \mathrm{G}$ licenses in Germany and the United Kingdom and, more recently, the auction of Advanced Wireless Services in the United States. These auction data demonstrate that the industry is willing to pay substantial amounts (in billions of dollars) for exclusive licenses. They also provide evidence of a high degree of spectrum scarcity and the industries' perceived advantages of offering new wireless services with new technologies. 
Table 1.1: 3G Spectrum Auction Results of Germany and the U.K.

\begin{tabular}{l||c||l||c}
\hline \multicolumn{1}{l||}{ Operator } & $\begin{array}{c}\text { Final Bid Price } \\
\text { (billions of USD })\end{array}$ & Spectrum Band & Dollars per MHz-POP \\
\hline \hline Germany & & & \\
T-Mobile & 10.26 & $2 \times 10 \mathrm{MHz}, 5 \mathrm{MHz}$ & $\$ 6.22$ \\
Vodafone D2 & 10.20 & $2 \times 10 \mathrm{MHz}, 5 \mathrm{MHz}$ & $\$ 6.18$ \\
E-Plus 3G & 10.14 & $2 \times 10 \mathrm{MHz}, 5 \mathrm{MHz}$ & $\$ 6.15$ \\
O2 (Germany) & 10.20 & $2 \times 10 \mathrm{MHz}$ & $\$ 6.18$ \\
MobilCom Multimedia & 10.14 & $2 \times 10 \mathrm{MHz}, 5 \mathrm{MHz}$ & $\$ 6.15$ \\
Quam (Group 3G) & 10.14 & $2 \times 10 \mathrm{MHz}, 5 \mathrm{MHz}$ & $\$ 6.15$ \\
\hline United Kingdom & & & \\
Hutchison 3G & 7.71 & $2 \times 15 \mathrm{MHz}, 5 \mathrm{MHz}$ & $\$ 4.30$ \\
Vodafone & 10.48 & $2 \times 15 \mathrm{MHz}$ & $\$ 5.84$ \\
O2 & 7.08 & $2 \times 10 \mathrm{MHz}, 5 \mathrm{MHz}$ & $\$ 5.92$ \\
T-Mobile (UK) & 7.04 & $2 \times 10 \mathrm{MHz}, 5 \mathrm{MHz}$ & $\$ 5.89$ \\
Orange 3G & 7.20 & $2 \times 10 \mathrm{MHz}, 5 \mathrm{MHz}$ & $\$ 6.02$ \\
\hline
\end{tabular}

${ }^{1}$ We ignore the unpaired spectrum since bidders place little value on the unpaired spectrum [2].

Note: Both auctions were held in 2000. License period is 20 years for both countries.

Germany has a population of 82.5 million and the UK has a population of 59.8 million.

Sources: [3], [4], and [5].

Table 1.2: Top 10 Bidders in Spectrum Auction of Advanced Wireless Services in the U.S.

\begin{tabular}{l||c}
\hline Bidder & $\begin{array}{c}\text { Final Bid Price } \\
\text { (billions of USD) }\end{array}$ \\
\hline \hline T-Mobile & 4.18 \\
Verizon Wireless & 2.81 \\
SpectrumCo (Comcast, Time Warner, Sprint Nextel, etc.) & 2.38 \\
MetroPCS & 1.39 \\
Cingular & 1.33 \\
Cricket (Leap Wireless) & 0.71 \\
Denali Spectrum (Also by Leap Wireless) & 0.37 \\
Barat Wireless (U.S. Cellular) & 0.17 \\
AWS Wireless & 0.12 \\
Atlantic Wireless & 0.10 \\
\hline
\end{tabular}

Note: The auction was held in 2006. License period is 15 years.

The total bid is 13.88 billion dollars. The average dollars per MHz-POP is $\$ 0.53$.

Source: [6]. 


\subsection{MOTIVATION}

Explosive demand for spectrum-based applications and technological advances in spectrumbased devices are the main driving forces of spectrum policy reform. In the United States, the FCC, in its Spectrum Policy Task Force (SPTF) report [7], has determined to evolve its spectrum policies towards more flexible and market-oriented approaches with the goal of providing incentives for spectrum users to employ technologically innovative and economically efficient uses of spectrum.

Measurement studies of the spectrum use in the SPTF report indicate that portions of spectrum are not in use in many geographical areas for significant periods of time. The sporadic use of spectrum is due to the variation of operations of existing spectrum users over time and the geographical separation among existing users. In response to this, the FCC has taken steps to facilitate the development of secondary markets ${ }^{1}$ for spectrum usage rights to permit spectrum to flow freely among users in response to economic demand [8]. The secondary markets would allow incumbent spectrum users to lease unused portions of their assigned spectrum to third parties who could put them into a better use. If the trade can be conducted with transparency and accountability, the spectrum trading may impose a clear, market-based opportunity cost upon incumbents, thereby providing them with incentives to conserve spectrum. The development of secondary markets includes the markets for license trading and for temporary access of existing licensed spectrum. This dissertation focuses on the secondary use of spectrum defined as a temporal use by a user of licensed spectrum owned by an incumbent who is distinct from the user.

Although the FCC is working on streamlining the administrative processes to encourage secondary use of spectrum, there are a number of factors that might dampen incumbents' incentives to share unused portions of spectrum. The outline of the issues and some of the research results are discussed in our previous work $[9,10,11,12]$. They are presented in Chapters 5-7.

\footnotetext{
${ }^{1}$ Secondary spectrum market is a market in which spectrum license holders can resell their spectrum usage rights.
} 


\subsection{PROBLEM STATEMENT}

The theme of this dissertation is on the development of secondary use of radio spectrum. The goal is to identify and study issues that are needed to be resolved for secondary use of spectrum to become feasible. In this dissertation, a primary spectrum user is defined as an incumbent spectrum user who holds one or more exclusive licenses, and a secondary spectrum user is referred to a spectrum user who temporarily leases portion(s) of spectrum from the primary user. The possibility of spectrum sharing depends on several technical, institutional, economic, and strategic issues from both sides of the lease. Some types of secondary use of spectrum are potentially suitable in some environments and for some wireless services and

not in others. It is thus necessary to evaluate the characteristics of both the primary and the secondary users in a variety of scenarios. Accordingly, the problem statement is given by the following key research questions:

- Why would a primary user want to share portions of his/her spectrum for secondary use? What are the conditions?

- Why would a spectrum user want to become a secondary user? What are the conditions?

The outcome of this dissertation will assist policy makers to create interventions (i.e., policy instruments) in order to obtain the favorable outcomes of spectrum management. In particular, it will enlighten the reality of secondary use and is a crucial step towards the development of secondary spectrum market for a more efficient use of radio spectrum.

\subsection{DISSERTATION OUTLINE}

The remainder of this dissertation is organized as follows: Chapter 2 provides a literature review of spectrum sharing approaches. Chapter 3 presents the theoretical framework and research tool including the transaction cost economics theory and the agent-based computational economics. Building upon the foundation of Chapters 2 and 3, Chapter 4 elaborates the research design, research questions, and experiments. Chapter 5 identifies issues in sec- 
ondary use of radio spectrum. The development and specifications of the agent-based model and the results and discussion of statistical testings are presented in Chapters 6 and 7, respectively. Finally, Chapter 8 concludes this dissertation and discusses the future research. 


\subsection{SPECTRUM SHARING TECHNIQUES}

In an attempt to improve efficiency of spectrum usage, researchers have identified multiple approaches to enable spectrum sharing between primary and secondary users. This chapter presents a review of current literature in spectrum sharing techniques and their associated issues. Section 2.1 describes how spectrum can be subdivided into portions for sharing. Section 2.2 provides a general classification of sharing techniques. Sections 2.3 and 2.4 review techniques and issues of cooperative and coexistent sharing approaches, respectively. Finally, Section 2.5 summarizes the current literature.

\subsection{SPECTRUM DIMENSIONING}

When considering access to radio spectrum, policy makers and system designers need to define a set of parameters that spectrum-based devices can be changed in order to access different parts of spectrum. It is, however, not clear how spectrum should be subdivided as one does not want to over-specify parameters and create unnecessary complexity. On the

other hand, defining too few parameters could undermine the ability of new technology to access some parts of spectrum, and hence result in parts of spectrum remains idle.

Spectrum license is primarily defined over geographical area, frequency range, and power. The FCC has recognized that this traditional definition of spectrum rights limits the full use of spectrum, and is considering the addition of time dimension to allow more dynamic allocation and assignment of spectrum usage rights [7].

Identifying dimensions of spectrum (i.e., defining spectrum space) is not only important to the development of spectrum regulation to allow innovative approaches of spectrum 


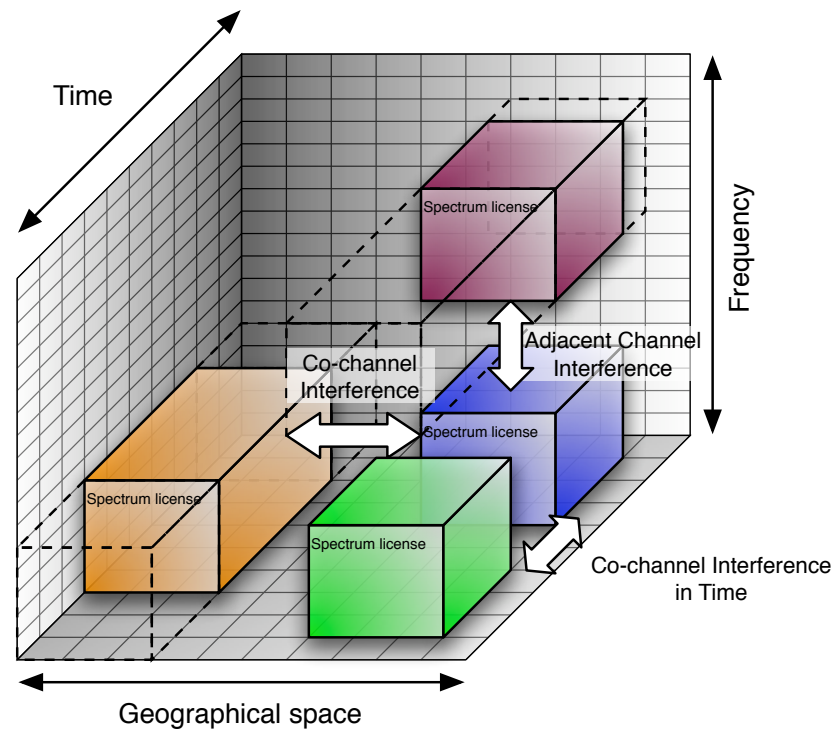

Figure 2.1: Traditional interference management shown in a three-dimensional spectrum space. The interference management is illustrated as reserving disjoint subspaces for exclusive use; with gaps in all dimensions to keep the acceptable level of interference.

access, but also provide basis to define rights and responsibilities for using spectrum. Several researchers have proposed models that capture multiple dimensions of radio spectrum $[13,14]$. The spectrum space is typically defined as an $n$-dimensional hyperspace in which dimensions are orthogonal. Each dimension can be divided to provide multiple-access to the spectrum space. In other words, each wireless signal can be uniquely identified by an $n$-dimensional vector $\mathbf{V}=\left\langle v_{1}, v_{2}, \ldots, v_{n}\right\rangle$, and non-interfering signals $\left(\mathbf{V}_{\mathbf{1}}\right.$ and $\left.\mathbf{V}_{\mathbf{2}}\right)$ will occupy different points in the spectrum space, $\mathbf{V}_{\mathbf{1}} \neq \mathbf{V}_{\mathbf{2}}$ (see Figure 2.1). Table 2.1 lists the spectrum dimensions proposed by researchers. This concept, however, is theoretical and is based on several assumptions such as ideal transmitters, ideal receivers, and negligence of signal propagation. In the real world, each dimension has its practical limitation as noted in the table. It should be also noted that not all dimensions are necessarily orthogonal (e.g., modulation and coding scheme), but they can still be used to distinguish signals through probabilistic methods. 
Table 2.1: Dimensions of Radio Spectrum Space

\begin{tabular}{|c|c|c|}
\hline Dimensions & Parameters & Notes \\
\hline Frequency & Frequency & $\begin{array}{l}\text { - Subdivision of too narrow frequency bandwidth } \\
\text { could result in unreasonable requirements for filter and } \\
\text { Doppler effect. } \\
\text { - In some cases, systems that utilize underlay transmis- } \\
\text { sions can coexist with other spectrum users in the same } \\
\text { frequency range. }\end{array}$ \\
\hline \multirow{3}{*}{ Geographical space } & Latitude & \multirow{3}{*}{$\begin{array}{l}\text { - Spectrum users cannot practically terminate the prop- } \\
\text { agation of signal right at the physical boundaries. Thus, } \\
\text { subdivision of physical space needs to consider the sig- } \\
\text { nal propagation of the systems and environmental condi- } \\
\text { tions in the area as they directly affect the geographical } \\
\text { coverage. }\end{array}$} \\
\hline & Longitude & \\
\hline & Elevation & \\
\hline Time & Time & $\begin{array}{l}\text { - Subdivision of time influences the degree of coordi- } \\
\text { nation needed. The smaller time scale (in ms or ns) } \\
\text { implies a closer and more complicate coordination (syn- } \\
\text { chronization) between users. }\end{array}$ \\
\hline \multirow{2}{*}{ Signal direction } & $\begin{array}{l}\text { Horizontal angle } \\
\quad \text { (azimuth) }\end{array}$ & \multirow{2}{*}{$\begin{array}{l}\text { - Although it is similar to the geographical division, this } \\
\text { dimension focuses on the benefits of directional antennas } \\
\text { that permit angular subdivision of angle-of-arrival. } \\
\text { - Given a single transmitter-receiver pair, there is no } \\
\text { practical method to generate multiple signals that ap- } \\
\text { pear to the receiver to be coming from different direc- } \\
\text { tions. } \\
\text { - Signal cannot be perfectly confined to a particular } \\
\text { angle due to the effects of multipath propagation. } \\
\text { - Transmissions between a pair of transmitter and re- } \\
\text { ceiver may not be limited to only a single direct path. } \\
\text { Advanced technologies (e.g., space-time coding) can ex- } \\
\text { ploit multipath to create multiple independent channels } \\
\text { between a transmitter-receiver pair. }\end{array}$} \\
\hline & Vertical angle & \\
\hline \multirow{2}{*}{$\begin{array}{l}\text { Transmission } \\
\text { characteristic }\end{array}$} & $\begin{array}{l}\text { Modulation/coding } \\
\text { scheme }\end{array}$ & \multirow{2}{*}{$\begin{array}{l}\text { - These can be viewed as secondary dimensions of radio } \\
\text { spectrum, used as information bearing parameters and } \\
\text { logical channelization. They can be distinguished by } \\
\text { their properties or by probability. } \\
\text { - There is no modulation or coding scheme that are } \\
\text { orthogonal to all other schemes. Thus, system design- } \\
\text { ers cannot independently choose a particular modula- } \\
\text { tion/coding and be certain that they will be immune to } \\
\text { signals from other systems employing different schemes. }\end{array}$} \\
\hline & Polarization & \\
\hline
\end{tabular}




\subsection{TAXONOMY OF SPECTRUM SHARING}

There are two fundamental approaches in spectrum sharing. Spectrum-based devices can coexist or cooperate to utilize the same spectrum space. In cooperative sharing model, spectrum-based devices communicate with each other using a common protocol to coordinate

spectrum access. For example, devices could form self-organizing wireless mesh networks and utilize the same spectrum space. Multiple wireless systems could create a common pool of spectrum and enable sharing through an established protocol (i.e., spectrum pooling). Spectrum leasing is also considered a cooperative model. In traditional lease, licensees need to negotiate terms and conditions with the lessees. In short-term lease or spot market, the negotiation among devices has to take place using a designated protocol to enable real-time spectrum leasing. Cooperative sharing approaches are further discussed in Section 2.3.

With coexistent sharing, explicit communications between systems do not exist. Unlicensed spectrum is a prominent example of the coexistence model. Successful products that use the unlicensed band such as IEEE 802.11 (WiFi), IEEE 802.15 (Bluetooth), and cordless phones do not communicate across systems. In other words, WiFi devices and Bluetooth devices do not decode each other's transmissions to operate in the unlicensed band. They may, however, detect the presence of other's transmissions in order to avoid collision. In the coexistence model, the importance is placed on the development of rules that govern operations of devices the shared spectrum space. These rules or protocols are known as spectrum etiquette and are designed according to characteristics of applications in the shared spectrum space. Section 2.4 presents coexistent sharing techniques in detail.

Taxonomy of spectrum sharing is built around cooperative and coexistent sharing approaches. Peha [15] offers policy options based on spectrum sharing from primary users and secondary users' point of view. Tables 2.2 and 2.3 summarize anticipated spectrum access approaches versus application requirements of primary and secondary users, respectively. 
Table 2.2: Spectrum Access Approaches of Primary Users

\begin{tabular}{l||l}
\hline Application Requirements & Spectrum Access Approaches \\
\hline \hline Guaranteed QoS & Exclusive licenses \\
$\begin{array}{l}\text { Guaranteed QoS, cooperate } \\
\text { with other primary users }\end{array}$ & Spectrum pooling \\
$\begin{array}{l}\text { No guarantee, cooperate } \\
\text { with other primary users }\end{array}$ & Cooperative wireless mesh networks \\
$\begin{array}{l}\text { No guarantee, coexist } \\
\text { with other primary users }\end{array}$ & Unlicensed spectrum \\
\hline
\end{tabular}

Table 2.3: Spectrum Access Approaches of Secondary Users

\begin{tabular}{l||l}
\hline Application Requirements & Spectrum Access Approaches \\
\hline \hline $\begin{array}{l}\text { Guaranteed QoS, cooperate } \\
\text { with primary user }\end{array}$ & $\begin{array}{l}\text { Secondary use; } \\
\text { Primary user guarantees QoS }\end{array}$ \\
$\begin{array}{l}\text { No guarantee, cooperate } \\
\text { with primary user }\end{array}$ & Interruptible secondary use \\
$\begin{array}{l}\text { No guarantee, coexist } \\
\text { with primary user }\end{array}$ & Opportunistic access \\
\hline
\end{tabular}




\subsection{COOPERATIVE SHARING TECHNIQUES}

This section describes four approaches of cooperative sharing. Section 2.3.1 presents spectrum trading and leasing, which is the most relevant literature to this dissertation. Sections 2.3.2-2.3.4 discuss other approaches of sharing including interruptible sharing, spectrum pooling, and virtual network operator.

\subsubsection{Spectrum Trading and Leasing}

The argument behind market-based spectrum policy has been that the market is a more efficient way to allocate scarce resources than external regulations. The market creates an environment in which the resources are transferred to the person who values them most, thereby creating an incentive for current owners to make efficient use of the resources. Spectrum auctions are one method towards the market-based policy, but they do not allow spectrum to move freely as licenses are still bound to a specific service or application. A solution to this problem is to set up licenses without restrictions of use and to establish a free market where license owners can sell or lease spectrum to others.

Not all markets, however, work perfectly. There are a number of factors that could result in market failures. One such failure that is significant in the case of spectrum is negative externality (i.e., interference) that could arise from energy spillovers into adjacent frequencies, geographical areas, or spillovers in other spectrum dimensions. The effects of externality can be controlled by definition of rights to use spectrum in terms of technical restrictions regarding power, frequency, place of operations, and limits in other dimensions. As a result, critics maintain that it is essential to clearly define spectrum property rights $[16,17,18,19]$.

How does one define property rights to spectrum resource? Researchers have been relating spectrum to land property and treating spectrum as private property, hence termed spectrum property right $[20,21]$. The foundation of this analogy is that both land and spectrum are scarce resources and the price system with property rights provides efficient

allocation. In the property rights regime, prime spectrum (i.e., frequencies below $3 \mathrm{GHz}$ ) is 
regarded as "beachfront property" and the owner has rights of exclusivity and transferability. Exclusivity means that no one can access the property without permission. Hence, causing interference is a use without consent or trespassing and will be subjected to penalties. The owners can use spectrum however they want, keep it indefinitely, or sell or lease to others (transferability rights).

De Vany et al. [21] and Matheson [22] propose definitions of property rights for spectrum use. The dimension of property rights follows the dimension of the radio spectrum space in Section 2.1. The idea is to confine signal radiation within a licensed spectrum space and allow only very low radiation outside the licensed region. Signals outside the region must be limited below a specified spectral power flux density, say, $x$, in watts $/ \mathrm{m}^{2} / \mathrm{MHz}$. With this definition of rights, there are no restrictions on types of applications, services, transmitter power, modulation, antenna height, and so forth, as long as the signal is kept below $x$ in every dimension. There are, however, a number of problems, mainly of a technical nature, that complicates practical implementation of this definition. Hatfield and Weiser [23] provide a comprehensive review of the complexity of the property rights model. The following discusses general problems with spectrum property rights and its definition.

- Division of spectrum space in any dimension may not be practical in the real environment. For example, arbitrary division of geographical space may not correspond to achievable coverage area since different frequencies yields distinct propagation characteristics in various types of terrains. Division of the time dimension into very short time slots may result in systems that are not properly synchronized. Similarly, division into very narrow frequency bandwidth may produce unreasonable filter requirements and Doppler effect. In the dimension of signal direction, partition of angle-of-arrival may not be accomplished due to lack of adequate narrow beam-width antenna and unavoidable scattering of radio wave.

- In the spectrum space, some degree of spillovers in many dimensions are unavoidable. This makes its analogy to the property system fails to some extent [15]. Propagation of radio wave is not entirely predictable. The field strength in a particular location is usually a sum of many multipath signals. These multipath components are fluctuating and may sometimes produce overall field strength that are larger than the limit. Re- 
searchers propose that definition of field strength limit in the spatial dimension should include statistical parameter to take into account the unpredictable variations of field strength due to multipath signals [21, 22]. It is unclear, however, how and what value this parameter should be defined.

- In the frequency dimension, transmitter can radiate signal inside the licensed bandwidth and keep signal below $x$ outside the licensed band. If this rule is enforced in all spatial locations, the transmitter must drop signal below $x$ very rapidly over a small change of frequency. This absolute limit $x$ may not be practical especially in the location very close to the transmitter where the field strength is very high [22]. Another phenomenon that may cause problems in the frequency domain is the effect of intermodulation in which two radio signals of different frequencies are mixed together, forming additional signals of different frequencies. The extra signals could interfere other systems that are not even in the adjacent frequency bands.

- In the time dimension, synchronization mechanism is a key to maintain the transmission within the licensed time slot. Spillovers, however, are still possible due to the propagation delay of the signal and power ramp-up and ramp-down delays of a transmitter.

- Another major limitation of using the spectrum space is the assumption of ideal receivers. An ideal receiver can theoretically separate signals that locate on different spectrum space coordinates. In reality, some spectrum space dimensions are easier to differentiate than others. A non-ideal simple receiver can easily separate two signals that are geographically far apart and reject other signals in distant frequencies. It may, however, have difficulties in separating signals with different angle-of-arrival. In fact, the ability to reject unwanted signal is dependent on the quality of the receiver. When interference occurs at the receiver, there is no definite rule that it is caused by offensive transmitters or by poor receiver performance. Accordingly, researchers and regulators support the need for the development of minimum receiver standards [7, 22]. The definition of technically and economically adequate receivers is still questionable.

- In the spectrum property rights regime, resolving disputes about spectrum use can become problematic $[18,24]$. Radio signals from multiple sources can aggregate and cause interference to other parties. One interfering signal from a particular user may not exceed 
the limit, but the accumulation of signals from all users may. As a result, it is not clear who is liable for interference. Also, unlike land and other natural resources, identifying the source and measuring the magnitude of interference may be a complicated task especially in the presence of a large number of spectrum users [21]. These issues could make the spectrum property regime difficult to administer and generate high enforcement cost.

- Spectrum users must tolerate some degree of interference due to the characteristics of radio waves as mentioned earlier. There is, however, no universal and comprehensive definition as to what constitutes acceptable interference and what describes harmful interference. It is likely that these definitions are highly dependent on applications and services of the spectrum users as well as the receiver standard.

- Flexibility on types of applications and service could inhibit the adoption of standards, which creates substantial problem for spectrum use unlike the zoning restrictions of land properties $[15,18,25]$. In many situations, such as television and radio broadcast, a single standard ensures compatibility and leads to greater benefits from economies of scale in manufacturing of standardized equipments.

- Some unconventional uses of spectrum may not easily fit in with the spectrum property regime. For example, underlay transmission (i.e., ultra wideband) - using a small amount of power and operating in the same frequency bands as existing communications without producing significant interference - is a promising technology to deliver a high data rate; however, its controversial operation is oftentimes viewed as incompatible with the spectrum property rights [15]. Hence, strictly defined dimension of the spectrum property may cause trouble for the deployment of innovative technologies.

- Similar to the standardization problem, there is an international issue in the spectrum management [25]. First, flexibility of spectrum property creates difficulties, if not impossible, to follow country borders and/or providing a cross-border service without rigorous international coordination. Second, it may prevent standardization of equipment and systems for international services.

In addition to the spectrum property right, transaction costs associated with moving spectrum from less efficient to more efficient use is another significant factor. It is generally agreed among researchers that transaction costs are central to the success of spectrum trading 
and leasing $[7,16,18,26]$. Transaction costs include the expenditure of resource and time for a secondary user to obtain spectrum access right from the primary user. The magnitude of transaction costs will determine the efficiency of this sharing scheme.

Peha and Panichpapiboon [27] take an initial step to quantitatively assess the secondary use of spectrum. In their model, the primary user is a GSM-based cellular operator who wants to share spectrum of the downlink channels (i.e., communications channels from a base station to mobile devices). The secondary user is prescribed to be a stationary user such as fixed broadband wireless networks or point-to-point wireless links. The model imposes two constraints: the secondary users must not interfere with the primary users and vice versa. With assumptions of GPS-capable devices, software-defined radio, and cooperation mechanism, both parties can coordinate their locations and adjust their frequency assignments to avoid interference. In these particular settings, they were able to demonstrate the financial incentive of the GSM operator for secondary usage.

Other studies focus on the economic aspect of spectrum trading. Leese et al. [28] and Nolan [29] provide similar models to investigate the potential economic effects of spectrum trading among firms. The outputs of firms (e.g., the number of subscribers in the case of GSM operators) are determined by the quantity of spectrum they own. The model confirms that spectrum trading between firms with a homogeneous service can increase efficiency by transferring spectrum licenses to the firm that values them most. Trading between firms with heterogeneous services is more complex because it involves a higher degree of interference coordination. Thus, the costs of reaching agreement and strategic behavior of firms can be significant. The results also show that the initial spectrum assignments among firms significantly determine trading equilibrium that arises.

\subsubsection{Interruptible Spectrum Sharing}

There are some spectrum incumbents whose spectrum use is highly unpredictable. Such spectrum users are not willing to participate in spectrum trading or leasing as the demand for spectrum access may arise after a spectrum sale or during a lease. A major spectrum user of this type is a public sector user. Public safety spectrum users such as police, firefighters, 
and paramedics, require reliable wireless communications in the event of an emergency. The amount of spectrum that is allocated and assigned to public safety typically corresponds to the worst-case scenario. Marsh [30] shows that the average usage is, however, less than 35\% of the system capacity, and even at $5 \%$ in the rural areas. Thus, several researchers assert that granting the ability to lease spectrum of public safety can provide significant advantages for both public safety and commercial users [16, 30, 31]. A sharing method for this type of users must be able to preempt or interrupt the secondary users when the primary users need spectrum access. This interruptible sharing scheme is a subset of the spectrum trading and leasing in Section 2.3.1.

Bykowsky and Marcus [31] propose a cooperative method that allows the primary user to exercise rights to preempt or call back its lease when needed. Their mechanism requires the public sector user to have a combination of software-defined radio and a specialized beacon transmission. The primary user who wishes to allow secondary use during the period of low spectrum utilization will transmit a beacon signal in a specified frequency band. The beacon signal indicates whether and what frequencies are available for sharing at the moment. The secondary user has to repeatedly detect and decode the beacon signal in order to operate in the sharing band, and must cease its transmission immediately when it fails to detect the beacon signal. With this mechanism, the secondary user takes the risk of being interrupted at any time at the discretion of the primary user.

\subsubsection{Spectrum Pooling}

Most current communications networks have variations in spectrum usage in time and geographical area. Therefore, spectrum can be underutilized in one system while another system may experience a spectrum shortage at the same time and location. The spectrum pooling concept is proposed to take advantage of this characteristic. A spectrum pooling system represents the idea that multiple spectrum owners merge their assigned frequencies into a common pool, and the pool members share the spectrum through coordination. The concept is also referred to as dynamic spectrum allocation (DSA) scheme. A number of European research projects under the headings of DRiVE [32], OverDRiVE [33], and $\mathrm{E}^{2} \mathrm{R}$ 
[34] have been investigating the potential of the DSA scheme. The DRiVE and OverDRiVE projects primarily focus on the specification of spectrum sharing between the Universal Mobile Telecommunications System (UMTS) 3G cellular network and the digital video broadcasting terrestrial (DVB-T) network. The $\mathrm{E}^{2} \mathrm{R}$ project emphasis on designing infrastructure for the beyond-third-generation wireless environments in which radio spectrum is shared seamlessly across spectrum-based services [35]. These projects are, however, high-level studies of architectural design of reconfigurable devices and their supporting functions for the DSA scheme [36].

A similar approach is called spectrum brokerage. In this case, the access to the spectrum pool is controlled and coordinated by a centralized entity called a spectrum broker. The spectrum broker can be an automatic agent or an entity that owns spectrum licenses. Weiss and Jondral [37] demonstrate that with a certain degree of flexibility of the transmitted signal, the spectrum brokerage is feasible from a technical perspective. Kamakaris et al. [38] and Buddhikot and Ryan [39] provide initial studies on the spectrum broker mechanism in the context of cellular networks. The major difficulties are in the coordinated DSA mechanisms between systems with different transmission schemes (i.e., frequency-division, time-division, and code-division).

\subsubsection{Virtual Network Operators}

Sharing spectrum through sharing network infrastructure is the most common form of sharing at present. Unlike other forms of cooperative sharing, the primary users do not directly share access to their spectrum resource. Rather, they share access to their network infrastructure (i.e., sharing network capacity). This type of sharing typically requires secondary users to utilize spectrum-based devices that are compatible with the primary system. The prime example of this sharing scenario is in the cellular phone service providers.

The cost of providing cellular phone services is expensive, especially in 3G. Service providers need to invest in cost of spectrum, cost of building out the networks, and marketing and selling the services. Thus, it is imperative that the providers need to acquire subscribers as quickly as possible to start earning a return on their investments. This creates a strong 
incentive for providers to lease portions of their network capacity to value-added resellers called Mobile Virtual Network Operators (MVNOs). An MVNO is defined as a service provider who does not own spectrum licenses and does not have network infrastructure. However, it has a complete control of its branding, marketing, subscriber acquisition, billing, and customer service. The brand image and customized services allow MVNO to target specific market segments more efficiently than the network providers themselves.

There are several MVNOs operating in the U.S. market. For example, Sprint-as a cellular carrier - share its network with Virgin Mobile, Mobile ESPN, Disney Mobile, and Boost Mobile. Some MVNOs operate on a number of carriers. TracFone - as an MVNOhas relationships with multiple existing providers, using a combination of CDMA and GSM technologies depending on region.

Linsenmayer [40] studies the MVNO business model as a form of secondary markets for spectrum. The study suggests that MVNO could lead to segmentation of markets for wireless services into two layers: the network companies who only provide infrastructures and supports, and the service companies who focus on the relationships with end-users. The adoption of MVNO continues to increase worldwide due to its simplicity in technical coordination compared to other cooperative schemes.

\subsection{COEXISTENT SHARING TECHNIQUES}

As a counterpart of cooperative sharing, coexistent sharing operates on a non-cooperative basis between spectrum-based systems. At present, unlicensed spectrum access is a leading model of coexistent sharing and is discussed in Section 2.4.1. Opportunistic spectrum access is a futuristic coexistent model and is presented in Section 2.4.2.

\subsubsection{Unlicensed Spectrum Access}

Wireless systems using unlicensed spectrum do not incur licensing costs. The unlicensed access also eliminates the lengthy and complicated licensing process. Accordingly, new 
spectrum-based devices can be readily adopted and quickly deployed. A wide range of unlicensed devices such as wireless LAN devices (IEEE 802.11), Bluetooth devices (IEEE 802.15), cordless telephones, and garage door openers represents the proliferation of unlicensed spectrum access. The key attribute of the unlicensed band is the absence of the right to exclude. The spectrum space is shared among devices and each of them can transmit at will without coordination. Besides, unlicensed users have less incentive to conserve the free-to-use spectrum compared to those who are using the licensed spectrum. Hence, catastrophic interferences among unlicensed users may occur. This consequence is referred to as the tragedy of the commons; a term that describes how unpunished greed can lead to a shared resource being unusable. Thus, it is essential to have a mechanism to manage the shared access.

From the technical perspective, a set of rules or operation guidelines (i.e., spectrum etiquette) can be used to manage unlicensed access. These rules have been defined and exercised in a number of dedicated unlicensed bands in the United States including Industrial, Scientific and Medical (ISM) band, Unlicensed Personal Communications Service Devices (UPCS) band, Unlicensed National Information Infrastructure Devices (U-NII) band, and Millimeter Wave band. The protocols, however, have been designed according to the expected characteristics of applications in each of the designated bands.

Satapathy and Peha $[41,42]$ investigate the potential risk of the tragedy of the commons and offer some preliminary designs of etiquette from the technical standpoints. Recently, Lehr and Crowcroft [43] address the challenge of managing non-exclusive use of spectrum and propose a set of design principle for etiquette that will govern the shared spectrum access.

Underlay transmission is another option for unlicensed access in addition to the dedicated unlicensed bands. Ultra-wideband (UWB) transmission is the technology under the current debate. As the name suggested, UWB trades off a large amount of power for a large amount of frequency bandwidth. Therefore, its bandwidth spans a large fraction of the total spectrum frequency. With its very low power, UWB signals appear as underlaying noises at many frequency bands of primary licensed users. The applicability of UWB systems is, however, limited to short-range uses (e.g., less than 10 meters and serve as wireless connectivity 
between consumer electronic devices) due to strict power limits.

\subsubsection{Opportunistic Spectrum Access}

Opportunistic spectrum access is a coexistent technique that allows secondary users to access spectrum of primary users without causing interference and without coordination. Opportunistic spectrum users automatically adapt their transmissions to occupy spectrum space where no other systems are operating. Thus, this method can increase utilization of spectrum in parts that would otherwise remain fallow. The opportunistic access creates numerous research questions and generates its own set of research areas.

Two enabling technologies are Software-Defined Radio (SDR) and cognitive radio. Reconfigurability is the main feature that describes the SDR. SDR-based devices can adapt their operating parameters such as frequency range (multiband), air interface (multimode), modulation scheme, power, and so forth by making a change in software [44]. Cognitive radio - also referred to as spectrum agile radio or smart radio - is a technology that is built on SDR. Cognitive radio devices have abilities to sense its surrounding environment and perform real-time changes in its operating parameters to provide reliable and efficient use of radio spectrum $[45,46]$.

The research framework in opportunistic access has been largely put down by the Defense Advanced Research Projects Agency: neXt Generation Communications (DARPA's XG) program (see for example $[17,47,48]$ ). The basic approach of opportunistic spectrum access is given as follows:

- Spectrum sensing and characterization: Detect and predict spectrum access opportunities that will not interfere with the operation of licensed primary users. The identification process is regulated by policies, which determine when spectrum is considered available and specify possibilities of using the available spectrum.

- Adaptive spectrum access: Cognitive radio constantly monitors the spectrum and adapts its transmission waveform to exploit the idle spectrum.

The implementation of opportunistic spectrum access involves a variety of research areas including software-defined radio, digital signal processing, radio devices, data and knowledge 


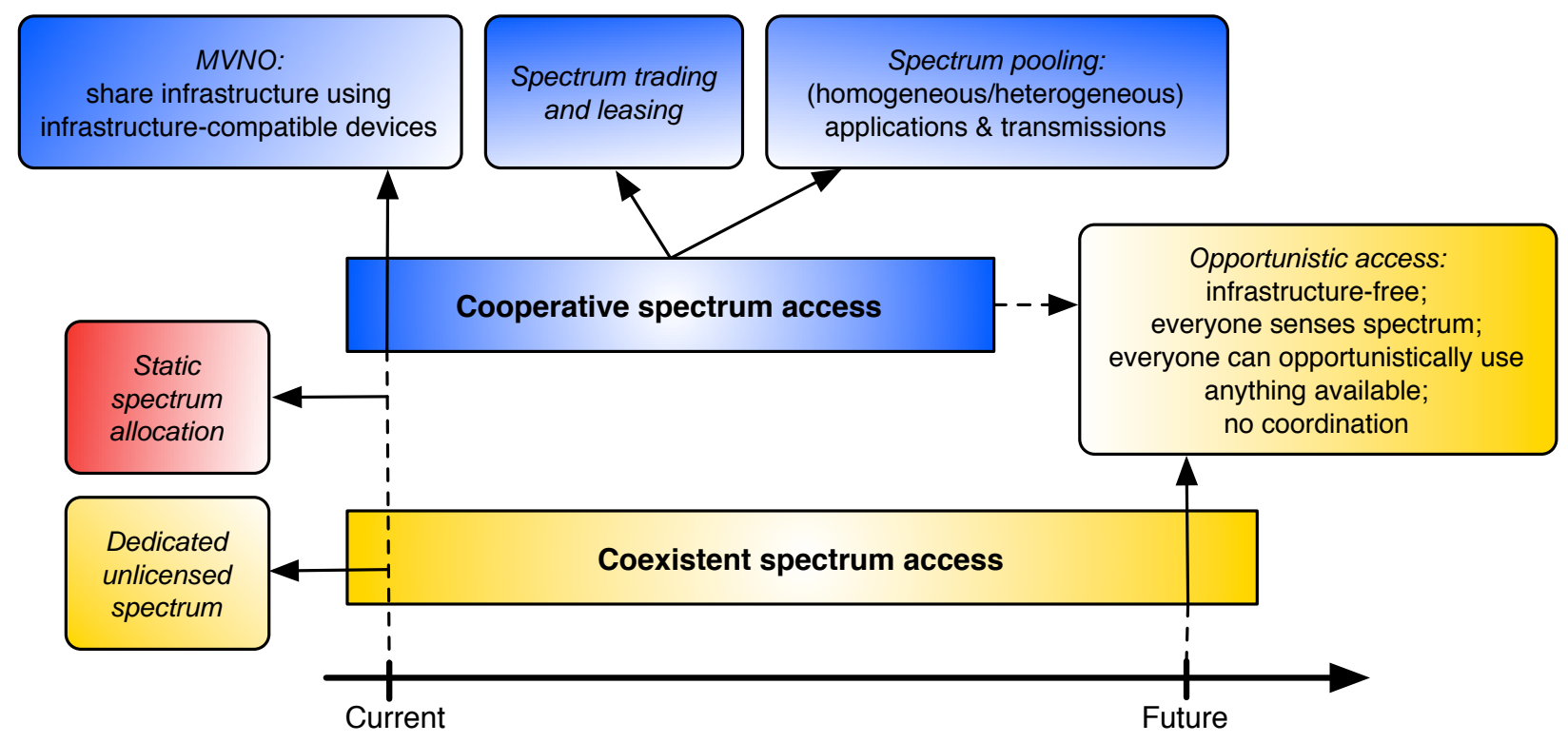

Figure 2.2: Evolution of spectrum sharing approaches.

representations and so forth.

\subsection{SUMMARY}

This chapter reviews the literature of spectrum sharing techniques between primary and secondary users. Under the cooperative sharing schemes, the studies on spectrum trading and leasing is very limited and mostly focuses on policy and regulatory aspects. The spectrum property right model has shown its technical complexity. Although preliminary studies has demonstrates the potential of spectrum leasing, an appropriate framework will need to address a combination of technical, economic, and strategic issues as well. Under the coexistent sharing, the opportunistic spectrum access generates a number of studies. Their emphasis is mostly on establishing the research framework and on technologies that will enable this ambitious form of spectrum sharing. However, the complexity of the sensing, 
agility, and protocol requirements at the spectrum-based devices is quite high. Thus, it is unlikely to be deployable in the near future. Figure 2.2 summarizes the current state of spectrum access and its evolution as envisioned by researchers in the field. 


\subsection{THEORETICAL FRAMEWORK AND COMPUTATIONAL TOOL}

This chapter reviews the theoretical framework and tool in this study. First, Section 3.1 introduces transaction cost economics (TCE) theory, its major assumptions, and assesses its key constructs. TCE is an underlying framework in this study of secondary use of spectrum. Next, Section 3.2 presents the concept of agent-based computational economics (ACE) which is used as a computational tool in this dissertation.

\subsection{TRANSACTION COST ECONOMICS (TCE)}

Transaction cost economics theory is an economic theory concerned with the analysis of buyer-supplier relationships using transaction costs as an argument. Here, the transaction cost is analogous to the economic equivalent of friction in the physical system. TCE was developed early by Ronald Coase [49] and was later greatly refined by Williamson [50, 51, 52]. The theory provides a set of principles for analyzing buyer-supplier transactions and determining the most efficient form of structuring and managing the transactions.

TCE uses the term "governance structure" to denote the organizational form in which a transaction between buyer and supplier can take place. Two polar forms are markets and firms (hierarchy). A transaction to obtain products or services can take place in markets (i.e., buy) or through coordination inside the firm (i.e., make). Thus, TCE is typically known as the study of make-or-buy decision. Between these two extreme forms, several intermediate forms exist including different types of contractual arrangement, alliances, and joint ventures. These intermediate governance structure are categorized as hybrid forms.

The preferred choice of organizational form obviously depends on the comparison of the 
transaction costs under each alternative. The theory provides a framework to assess the strengths and weaknesses of alternative forms under different circumstances through the analysis of transaction characteristics and behaviors of economic agents.

When comparing the uses of markets and firms to organize a transaction, markets are characterized by the price mechanism based on demand and supply to achieve efficient resource allocation. Market price serves as a signal to inform market participants of potential exchanges that would leave them better off. The price signal provides powerful incentives for participants to recognize profit opportunities and allows them to adapt to changes in demand and supply as the price reflects the value of the trading commodity. The use of the price mechanism, however, incurs a number of inherent activities, which can be translated into transaction costs as follows:

- Search and information costs include gathering information about possible trade solutions, find potential trading partners and their quality of products or services. These are ex ante (i.e., before the transaction).

- Negotiation costs are another ex ante. They are resulting from negotiation over possible solutions and aligning conflicting interests of the transacting parties.

- Monitoring and enforcement costs are ex post transaction costs (i.e., after the agreement has been reached). Once the transaction is underway transacting partners have to ensure compliance with the specified terms and respond when the agreement is violated. The resulting costs are in forms of costs of inspection and auditing, costs of arbitration, and court fees.

According to Coase [49], the existence of these transaction costs through the use of price mechanism creates a resource burden and the firm exists because the costs of some specific transactions are lower when carry out inside the firm. Compared to a market, organizing the transaction under unified ownership offers greater administrative control, facilitates coordination, and creates higher protection for specific investments. Especially in the situations where adaptation to changes by coordination is significant, firms are more likely to outperform markets [53]. Although these benefits can reduce the transaction costs occurred in the market, they are countered by the added bureaucratic costs and weaker 


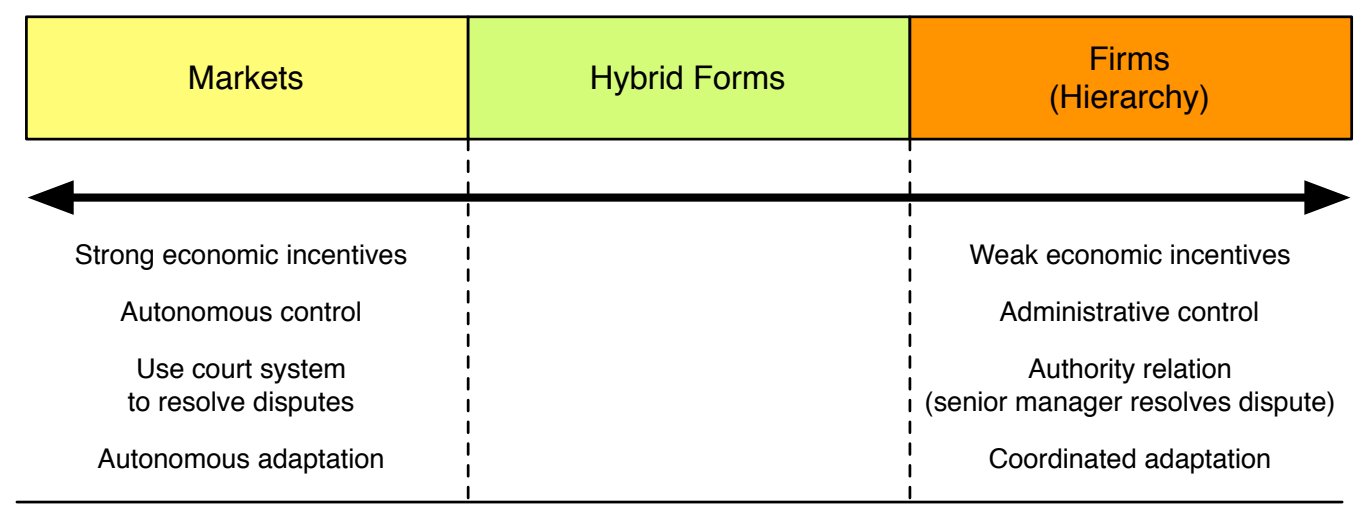

Figure 3.1: Governance structures in transaction cost economics.

economic incentives. The shift from the market to the hybrid form, and to the firm creates tradeoffs between stronger economic incentives and adaptive properties of the market, and stronger protections and coordination properties of the firm. The empirical studies show that a number of economic activities across various industries are generally aligned with this central theoretical framework of TCE $[54,55,56,57]$. Figure 3.1 illustrates a market-firm continuum of governance structures as described by TCE.

As developed by Williamson [50, 51, 52], TCE explains the decisions made by the trading partners on the choices of organizational structures using human behaviors and transaction characteristics. Two major behavioral assumptions underlying the existence of transaction costs are bounded rationality and opportunism. The following describes rationale behind these assumptions.

1. Bounded rationality refers to the limited capability of decision makers to be rational even they are intended to be. People have limited cognitive and information processing capabilities. This is contrast to neoclassical economics, which assumes perfect rationality. Due to this limitation, decision makers have imperfect foresight and cannot fully recognize potential hazards that might occur in the future. Accordingly, they cannot create contracts that will protect them against all possible events that might occur in a 
trade. Thus, TCE maintains that complex contracts are typically incomplete ${ }^{1}$. This is particularly problematic when transactions take place in uncertain environments. The incompleteness could expose transacting parties to transactional hazards from potential opportunism.

2. Opportunism refers to the assumption that transacting partners are driven by their selfinterest seeking behaviors and they have a potential to behave opportunistically in order to take advantages in a trade. According to TCE, opportunistic behavior explicitly refers to acting in bad faith including providing misleading information, creating confusion, violating agreements with the goal of taking advantages in the buyer-supplier relationship. It is considered that merely the possibility of opportunism will raise the transaction costs as transacting partners try to safeguard their investment from transaction hazards. Such an opportunistic behavior, however, can be discouraged by transacting under the proper organizational form, depending on the transaction characteristics.

The characteristics of the transaction determine the magnitude of transaction costs. In TCE, there are three principal attributes which affects the degree of transaction costs [51]:

1. Asset specificity is considered a key factor in TCE. Specific assets are valuable in the context of a specific transaction and have much lower value in the best alternative uses. They are likely to produce sunk costs when the relation between buyers and suppliers terminates prematurely ${ }^{2}$. Transactions that involve specific assets force economic agents to put higher safeguard into their investment. This type of transactions is particularly vulnerable to opportunism. On the other hand, non-specific assets pose fewer hazards, because buyers can easily switch to alternative sources, and suppliers can sell products or services to other buyers without difficulty.

2. Uncertainty refers to the possibility that unforeseen circumstances arise during the relation and the parties involving in the transaction need to adapt to. Transactions under

\footnotetext{
${ }^{1} \mathrm{~A}$ contract would be "complete" if the parties to an agreement could specify their respective rights and obligations for every possible future state of the world.

${ }^{2}$ The most popular example has been the relationship between General Motors and Fisher Auto Body. At the time, auto bodies were produced by using a specific machine to stamp the metal into its required shape. The machine was specific to individual car design. Fisher could hold up GM by demanding monopoly prices, because stopping production was extremely costly to GM who could not obtain auto bodies from other sources on short notice.
} 
relatively high uncertainty environments require buyers and suppliers to be aware of the potential complication and subsequent costs of adaptation. Thus, a higher level of uncertainty often makes contracting difficult and produces higher transaction costs, as both parties will spend more effort to monitor the transaction. Uncertainty also introduces the risk of opportunism during the adaptation process.

3. The frequency in which the transaction occurs can influence the appropriate organizational choice. Transaction that occurs rarely may not worth investing in an expensive form of organization.

These attributes of the transaction are used as proxies to analyze transaction costs in empirical studies, because direct measurement of transaction costs is difficult if not impossible. The combination and interaction of these three attributes serve to justify the appropriate form of organization to carry out the transaction.

In summary, TCE uses transaction costs to provide the key to understanding alternative forms of organization and contractual arrangement. The main focus is on the relative costs of conducting transactions in one organizational form relative to others. Therefore, what matters is not the absolute value of transaction costs, but rather the relative ranking of the costs associated with different organizational forms or contractual arrangement. Other details of TCE theory are beyond the scope of this study.

\subsection{AGENT-BASED COMPUTATIONAL ECONOMICS (ACE)}

ACE is a computational study of economies modeled as dynamic systems of autonomous interacting agents [58]. The term "agent" refers to an entity in a computational world and is described by its behavior and function. In ACE, agents can represent a broad range of entities such as individuals (e.g., consumers, producers, and intermediaries), groups of individuals (e.g., firms, agencies), other social and environmental entities (e.g., markets, infrastructure, and geographical area).

The merit of ACE is often compared with the mainstream, neoclassical approach in economics. With the neoclassical analysis, agents are typically assumed to be homogeneous and 
fully rational. The goal is largely on deriving analytic closed-form solutions (i.e., equilibria) of an economic system. Accordingly, ACE researchers often argue that this conventional analysis is a top-down and deductive approach. The analysis tends to oversimplify and represent stylized settings of the economic system and would only be suitable for the system where agents do not vary much in their characteristics.

While knowing the outcomes of the system is considered interesting, other important aspects could not be easily obtained from the conventional analysis. As Schelling [59] observed,

... there is nothing particularly attractive about an equilibrium. An equilibrium is simply a result. It is what is there after something has settled down, if something ever does settle down. The idea of equilibrium is an acknowledgement that there are adjustment processes; and unless one is particularly interested in how dust settles, one can simplify analysis by concentrating on what happens after the dust has settled.

It is the adjustment process that is considered more important to understand and, therefore, is the objective of the ACE approach. The goal of ACE is not to derive closed-form solutions, but rather to observe and study the aggregate outcomes and the norms of behavior that are developed and sustained over time. In contrast to the top-down deductive approach of neoclassical economics, ACE works from bottom up by creating adaptive, heterogeneous, and autonomous agents who interact with one another in dynamic environments. It is also common in the agent-based literature to assume that agents are bounded rational.

The tasks of ACE modeling start from specifying the initial attributes of the agents. The attributes of any agent may include its type, characteristics, behavioral methods, and internal information about itself and other agents. These agents are put together to represent the economic system. The system then evolves over time as a result of interactions among agents without any intervention from the outside. The agents in ACE are possibly making suboptimal decisions as a result of their limited cognitive capabilities and on the basis of their locally available information. The essence of this approach is to focus on the process in which the agents interact with each other and adapt their behavior based on their experiences. These observed processes and outcomes that emerge are used to understand the economic system.

The usefulness of ACE methodology has been shown in a diverse range of research topics. 
Social scientists use ACE to study the evolution of behavioral norms of individuals in the society $[60,61]$. The agent-based computational model is used to explain how patterned behavior can arise from interactions among agents. Bottom-up modeling of market processes is also one of the most active ACE research areas. The agent-based market model is used to predict and provide explanation for empirically observed data in stock markets and foreign exchange markets $[62,63,64]$. The ACE methodology is also applied to the restructured electricity markets to predict the consequences of implementing these new markets, which are considered difficult to analyze with conventional statistical and analytical tools due to the complexity of the electric power industry [65]. Several special journal issues have been devoted to investigate the use of ACE in the topics mentioned earlier as well as other samples of ACE research [66, 67, 68].

The weaknesses of ACE arise from the nature of the bottom-up modeling approach. The ACE model requires a complete specification of initial conditions including agent data, behavior, and institutional arrangement. As the system operates, the chain of causality that evolves the system over time tends to be highly complicated. Accordingly, the outcomes that emerge tend not to be direct consequences of the initial specifications of the agents and their environment. This loss of analytical tractability is observed as a drawback of the ACE approach. The ACE model is also conditional upon the specific values of the parameters used in the initial specifications. ACE modeler needs to be careful if the small changes in these parameters could dramatically affect the types of the outcomes. Intensive experiments using a wide range of initial specifications may be required to achieve robust prediction $[69,70]$. 


\subsection{RESEARCH DESIGN AND RESEARCH QUESTIONS}

Given the background of spectrum sharing techniques in Chapter 2 and the introduction to

theory and tool in Chapter 3, this chapter elaborates the merit of this research and the use of theory and tool in Section 4.1. Section 4.2 identifies research questions that will be analyzed by the agent-based model. Finally, Section 4.3 describes multiple sets of experiments to perform statistical testings.

\subsection{RESEARCH OUTLINE}

The focus of this dissertation is on the development of secondary use of radio spectrum. The secondary spectrum markets include the markets of license trading and for temporary access of existing licensed spectrum. It is the latter that defines secondary use and is the scope of this research. The review of the literature in Chapter 2 shows that the studies on secondary use are very limited. Most of them are in early stages and focus on a single aspect of the problem such as technical, economics, or policy and regulatory issues. We believe that an appropriate framework will need to address both technical issues and non-technical issues such as policy and business implementations as well. Accordingly, the theme of this dissertation is guided by the following key research questions:

- Why would a primary user want to share portions of his/her spectrum for secondary use? What are the conditions?

- Why would a spectrum user want to become a secondary user? What are the conditions? 
The objective is to identify and study issues that are needed to be resolved for secondary use to become feasible. The possibility of spectrum sharing depends on several technical, economic, institutional, and strategic issues from both primary users and secondary users. Central to the issues are the effects of transaction costs in executing secondary use spectrum sharing. A number of researchers express concern that the success of secondary use will largely depend on transaction costs and the ability of the sharing mechanism to minimize them $[7,16,18,26]$. Transaction costs associated with secondary use must be reasonable for spectrum sharing to become practical.

In this dissertation, we propose the use of transaction cost economics (TCE) as the theoretical framework to study transaction costs in secondary use of radio spectrum. In Section 3.1, TCE demonstrates that transaction costs depends largely on the choice of organizational form in which a transaction between buyer and supplier take place. In the context of secondary use, we expect that transaction costs depend on technical factors (such as types of wireless services, application requirements, and capabilities of radio devices) and economic factors (such as number of participants in the secondary use and price settings). Also, corresponding to TCE, we anticipate that different forms of secondary use could play an important role on the magnitude of transaction costs as well as the level of uncertainties due to potential interference between primary and secondary users. For example, a spot market, in which a secondary user obtains spectrum access on demand, might be appropriate only in certain circumstances. In other cases, it might be preferable to use more complex contractual arrangements such as a long-term leasing or an indirect spectrum access such as Mobile Virtual Network Operator (MVNO), in which the operations of primary and secondary users are tightly coupled. In short, we hypothesize that secondary users would emerge when transaction costs are low compared to alternative methods to obtain spectrum access.

We also propose the use of agent-based computational economics (ACE) as a computational tool in this study. We believe that the bottom-up modeling approach of ACE is appropriate for the problem of secondary use, which has a complex interrelated structure between primary and secondary users in both technical and non-technical issues; and partly due to the lack of empirical data on spectrum trading and leasing. Accordingly, ACE will 
be used to model the development of secondary use transactions. As discussed earlier in Section 3.2, ACE aims to observe the aggregate outcomes and understand the process that leads to the outcomes as a result of interactions among agents. The understanding of the process, in our case the conditions that lead to secondary use of spectrum, can then be used to create interventions (i.e., secondary use policy) in order to obtain the favorable outcomes of spectrum management.

The following describes more specifically the research deliverables of this dissertation in the remaining chapters.

- Chapter 5 identifies issues that are needed to be resolved for secondary use of spectrum to become feasible.

- Chapter 6 describes the effects of transaction costs in secondary use of radio spectrum and establishes a relationship between transaction cost economics theory and the secondary use. The focus is on identifying the possible organizational forms to carry out spectrum access transactions and the relationship among them.

- The development and specifications of the agent-based model for secondary use of radio spectrum are also presented in Chapter 6. The model integrates technical and economic considerations for spectrum access transactions. It also incorporates TCE behavioral assumptions that underlie the existence of transaction costs into agents that represent spectrum users in the model.

- Experiments on the model to predict the form and fashion with which the secondary use will develop are set out by various pre-conditions in the research questions in Section 4.2. The results and discussion of feasible technical parameters are given in Chapter 7 . We assume that both primary and secondary users employ infrastructure networks, and the primary users share spectrum of their downlink channels (i.e., communications channels from a base station to mobile devices).

- The solutions derived from the agent-based model will not be closed form solutions, but can be referenced to gather some insights as to how the spectrum leasing will work on an expansive macro level. The main contribution of this work is the agent-based model that can be utilized to form innovative and creative policy instruments for the secondary markets for spectrum access. 


\subsection{RESEARCH QUESTIONS}

The use of the agent-based model ${ }^{1}$ is guided by the following research questions:

- (Q1) Does the secondary use of spectrum only emerge when the number of participants in the market is high? In other words, given scenarios $i$ and $j$, if the total number of spectrum consumers $N_{C_{i}}^{\text {total }}>N_{C_{j}}^{\text {total }}$ and the total number of providers $N_{P_{i}}^{\text {total }}>N_{P_{j}}^{\text {total }}$, then the proportion of consumers selecting secondary use to the total number of consumers $\frac{N_{C_{i}}^{\text {secondary }}}{N_{C_{i}}^{\text {total }}}>\frac{N_{C_{j}}^{\text {secondary }}}{N_{C_{j}}^{\text {total }}}$.

- (Q2) Is the secondary use higher in rural areas (scenario $i$ ) than urban areas (scenario $j)$ ? More specifically, let $N_{T}$ denotes the number of transmitters per primary user, $N_{R}$ is the number of receivers per transmitter, and $A$ is the size of geographical area. If the path-loss exponent $\alpha_{p l_{i}}<\alpha_{p l_{j}}$ and the transmitter and receiver densities of the primary users $\frac{N_{T_{i}}}{A}<\frac{N_{T_{j}}}{A}$ and $\frac{N_{R_{i}}}{A}<\frac{N_{R_{j}}}{A}$, then the proportion of spectrum consumers selecting secondary use to the total number of consumers $\frac{N_{C_{i}}^{\text {secondary }}}{N_{C_{i}}^{\text {total }}}>\frac{N_{C_{j}}^{\text {secondary }}}{N_{C_{j}}^{\text {total }}}$.

- (Q3) Is the secondary use higher when primary users have consistent and predictable spectrum usage (e.g., constant or scheduled spectrum uses of radio or television broadcasts) compared to other cases (e.g., unpredictable uses of public safety spectrum users)? In other words, given scenarios $i$ and $j$, if the spectrum use of the primary users of the scenario $i$ is a periodic function and the scenario $j$ is an aperiodic function, then the proportion of consumers selecting secondary use $\frac{N_{C_{i}}^{\text {secondary }}}{N_{C_{i}}^{\text {total }}}>\frac{N_{C_{j}}^{\text {secondary }}}{N_{C_{j}}^{\text {total }}}$.

- (Q4) Do spectrum users that require larger coverage area obtain spectrum access with higher degree of control $^{2}$ than those that require smaller coverage area? In other words, given scenarios $i$ and $j$, if the maximum coverage $D_{\max _{i}}<D_{\max _{j}}$, then the average degree of control among spectrum consumers $\bar{d}_{i}<\bar{d}_{j}$.

- (Q5) Do spectrum users with rigid application requirements obtain spectrum access with higher degree of control than those with flexible application requirements? Assume that

\footnotetext{
${ }^{1}$ See Chapter 6 for the complete specifications of the model.

${ }^{2}$ See Section 6.2 for the definition of "degree of control" among spectrum access choices.
} 
the exponential utility function ${ }^{3}$ of the spectrum users is

$$
\mathcal{U}(\gamma)= \begin{cases}\alpha_{u}\left[1-\exp \left(-\left(\gamma-\gamma^{*}\right) / \eta\right)\right] & \text { if } \gamma \geq \gamma^{*} \\ 0 & \text { otherwise }\end{cases}
$$

where $\gamma$ is the received SINR, $\gamma^{*}$ is the SINR threshold value, and $\eta(>0)$ is the parameter that can be varied to obtain different levels of concavity and correspondingly different levels of application requirements ${ }^{4}$. In addition to the application requirements, different spectrum users may derive different utilities from the same SINR depending on their spectrum-based services. For example, public safety spectrum users are more sensitive to the received SINR compared to other spectrum users. Thus, $\alpha_{u}$ is used to capture user's sensitivity to the received SINR. With the above definitions, given scenarios $i$ and $j$, if $\eta_{i}<\eta_{j}$ and $\alpha_{u_{i}}>\alpha_{u_{j}}$, then the average degree of control among spectrum consumers $\bar{d}_{i}>\bar{d}_{j}$.

- (Q6) Does the expansion of unlicensed spectrum only benefit spectrum users with flexible application requirements and small coverage area? Given scenarios $i$ and $j$ with the bandwidth of unlicensed spectrum $U_{i}>U_{j}$, if the maximum coverage $D_{\max _{i}}<D_{\max _{j}}$ and $\eta_{i}>\eta_{j}$ from (4.1), then the proportion of spectrum consumers selecting unlicensed spectrum to the total number of consumers $\frac{N_{C_{i}}^{\text {unlicensed }}}{N_{C_{i}}^{\text {total }}}>\frac{N_{C_{j}}^{\text {unlicensed }}}{N_{C_{j}}^{\text {total }}}$.

\subsection{EXPERIMENTAL DESIGN}

To perform statistical testings on the research questions, we create four sets of experiments. The first set is designed to analyze research question Q1. Here, we perform experiments by varying the number of consumer and provider agents and measure the percentage of consumers selecting secondary use. The second set is designed to examine research questions Q2 and Q3 by measuring consumer's choice of spectrum access in rural and urban settings and that of different spectrum usage characteristics of primary users. The third set is created

\footnotetext{
${ }^{3}$ See Section 6.3 for discussions of the utility-based approach and the application requirements based on SINR.

${ }^{4}$ This utility function takes a shape of a step function as $\eta \rightarrow 0$.
} 
Table 4.1: Full Factorial Design for Q1

\begin{tabular}{c||l}
\hline Factors & Levels \\
\hline \hline$N_{C}$ & $\{1,3,5,7,9,11,13,15,17,19\}$ \\
$N_{P}$ & $\{1,3,5,7,9,11,13,15,17,19\}$ \\
\hline \# of experiments & $10 \times 10 \times 30=3000$ \\
\hline
\end{tabular}

to analyze research question Q4 by varying the consumer's coverage area and measuring the average degree of control among consumer agents. It can also be used to evaluate research question Q5 by testing on the rigid and flexible application requirements and consumer's QoS sensitivity. Finally, the fourth set is designed to analyze research question Q6. We measure the percentage of consumers selecting unlicensed spectrum in response to the amount of spectrum in the unlicensed band, the consumer's coverage area, and the rigid and flexible application requirements. Tables 4.1-4.4 summarize respectively factors used in full factorial design with 30 times replications.

In each experiment, we measure the means of $\frac{N_{C}^{\text {secondary }}}{N_{C}^{\text {total }}}, \frac{N_{C}^{\text {unlicensed }}}{N_{C}^{\text {total }}}, \frac{N_{C}^{\text {exclusive }}}{N_{C}^{\text {total }}}, \bar{d}$, and $\bar{l}$. Finally, Table 4.5 describes methods to perform statistical testings according to the experimental design. 
Table 4.2: Full Factorial Design for Q2 and Q3

\begin{tabular}{|c|c|}
\hline Factors & Levels \\
\hline $\begin{array}{c}N_{T} / A \\
\left(\text { transmitters per } \mathrm{km}^{2} \text { ) }\right. \\
\text { Corresponding coveraqe }\end{array}$ & $\begin{array}{l}\{0.0625,0.25,0.5625,1.0,1.5625\} \\
\{4.91 \%, 19.63 \% .44 .18 \% .78 .54 \%, 90.61 \%\}\end{array}$ \\
\hline $\begin{array}{l}N_{R} \\
\text { (receivers per transmitter) }\end{array}$ & $\{1,5,10,15,20\}$ \\
\hline$\alpha_{p l}$ & $\{2.0,3.5\}$ \\
\hline $\begin{array}{l}\text { Spectrum use of } \\
\text { primary users }\end{array}$ & 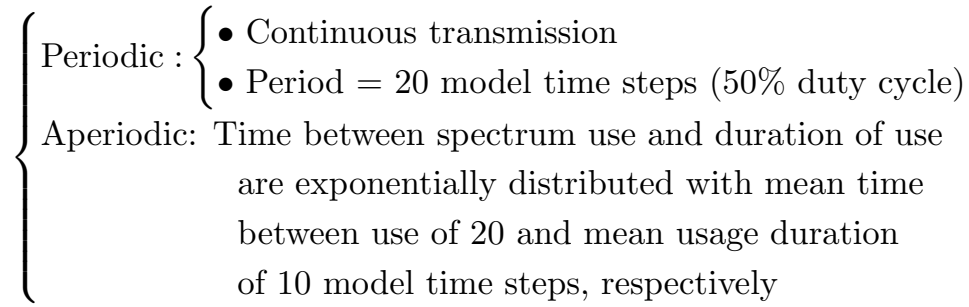 \\
\hline \# of experiments & $5 \times 5 \times 2 \times 3 \times 30=4500$ \\
\hline
\end{tabular}

Table 4.3: Full Factorial Design for Q4 and Q5

\begin{tabular}{c||l}
\hline Factors & Levels \\
\hline \hline$D_{\max }($ meters $)$ & $\{10,100,250,500,1000\}$ \\
$\left(\eta, \gamma^{*}\right)$ & $\{(0.001,15),(1.0,10)\}$ \\
$\alpha_{u}$ & $\{3,6,9,12\}$ \\
\hline \# of experiments & $5 \times 2 \times 4 \times 30=1200$ \\
\hline
\end{tabular}


Table 4.4: Full Factorial Design for Q6

\begin{tabular}{c||l}
\hline Factors & Levels \\
\hline \hline$U(\mathrm{MHz})$ & $\{10,50,100,150\}$ \\
$D_{\max }$ (meters) & $\{10,100,250,500,1000\}$ \\
$\left(\eta, \gamma^{*}\right)$ & $\{(0.001,15),(1.0,10)\}$ \\
\hline \# of experiments & $4 \times 5 \times 2 \times 30=1200$ \\
\hline
\end{tabular}

Table 4.5: Statistical Testing Methods

\begin{tabular}{c||c||c||l}
\hline Research Question & Dependent Variable & Independent Variables & Methods \\
\hline \hline Q1 & $N_{C}^{\text {secondary }}$ & $N_{C}, N_{P}$ & $\begin{array}{l}\text { Multiple regression with } \\
\text { two-way interaction and } \\
t \text {-test on the coefficients }\end{array}$ \\
\hline Q2, Q3 & $N_{C}^{\text {secondary }}$ & $\begin{array}{c}N_{T}, N_{R}, \alpha_{p l}, \\
\text { Spectrum use of } \\
\text { primary users } \\
\text { categorical variable) }\end{array}$ & $\begin{array}{l}\text { Multiple regression with } \\
\text { a dummy variable, } \\
\text { three-way interaction, } \\
\text { and } t \text {-test on the coefficients }\end{array}$ \\
\hline Q4, Q5 & $\bar{d}$ & $D_{\max },\left(\eta, \gamma^{*}\right), \alpha_{u}$ & $\begin{array}{l}\text { Multiple regression with } \\
\text { three-way interaction and } \\
t \text {-test on the coefficients }\end{array}$ \\
\hline Q6 & $N_{C}^{\text {unlicensed }}$ & $U, D_{\max },\left(\eta, \gamma^{*}\right)$ & $\begin{array}{l}\text { Multiple regression with } \\
\text { three-way interaction and } \\
t \text {-test on the coefficients }\end{array}$ \\
\hline
\end{tabular}




\subsection{ISSUES IN SECONDARY USE OF RADIO SPECTRUM}

This chapter establishes a set of questions and identifies issues relating to the development of secondary use. The creation of the spectrum markets for secondary use brings about many research questions necessary to be addressed in order to develop appropriate policies that

lead to a successful market $[9,10]$. The feasibility of the market depends not only upon technical feasibility of various technologies that would contribute various scenarios of how spectrum can be shared, it is also depend upon economics, regulatory, and political issues. Some of the issues that have been raised regarding secondary use of spectrum include:

- Capabilities of advanced radio technologies

- Regulatory issues relating spectrum leasing

- Rights, responsibilities, and enforcement problems

- Efficiency of the market

- Pricing and billing

- Secondary use in government frequency bands

- Government control

- Spectrum hoarding, speculation, and monopoly

- Concerns about public safety services

Instead of presenting an exhaustive list of issues, we attempt to outline what we believe as fundamental elements that would constitute the starting point of the secondary use of radio spectrum. These would allow us to incorporate miscellaneous issues as listed above and systematically perform a study. Sections 5.1-5.4 present four fundamental questions that are needed to be unfolded for the concept of secondary use to become reality. In this dissertation, we focus on the first two questions in Sections 5.1 and 5.2. 


\subsection{WHY WOULD A PRIMARY USER WANTS TO SHARE PORTIONS OF HIS/HER SPECTRUM FOR SECONDARY USE?}

For spectrum sharing to take place, current spectrum incumbents need to have some incentives to sell or lease their own spectrum. From economic and financial standpoints, Peha and Panichpapiboon demonstrate that a commercial cellular operator has a financial incentive to lease spectrum for secondary use [27]. However, the interest of trading the spectrum for secondary use largely depends on the classification of primary users ${ }^{1}$. For instance, spectrum users who are government agencies do not pay for the rights to access radio spectrum. Therefore, they tend to have fewer financial incentives to share their spectrum than those who have paid for the spectrum (i.e., commercial spectrum users). Strategic factors also play an important role among the commercial users. Existing commercial users of spectrum could have small incentives to sell or lease excess or unused spectrum if potential buyers will use their acquired spectrum to provide a service competing with the sellers.

In terms of technical issues, spectrum has multiple access dimensions, as described earlier in Section 2.1. Regardless of the dimensions of sharing, a lessee's bid and a licensee's offer must match in all dimensions for trading to occur. This could imply that the number of participants in the spectrum market may be low. This lack of liquidity decreases the likelihood that a trade takes place, leading to the need to develop policies, incentives, and market mechanisms that increase market liquidity and enhance the willingness of spectrum users to conduct a trade. The transaction costs of secondary use are still unclear. It is likely to be related to the negotiation process, which in turns depends on types of spectrum use and dimensions of sharing.

\footnotetext{
${ }^{1}$ See Section 1.3 for the definitions of primary users and secondary users.
} 


\subsection{WHY WOULD A SPECTRUM USER WANTS TO BECOME A SECONDARY USER?}

While it is clearly necessary to examine the incentives for spectrum sharing, it is equally important to consider under what circumstances potential secondary users would emerge. This answer, it turns out, depends in large measure on the application that the secondary users have in mind. In general, new spectrum users have several options to obtain access to spectrum as follows:

- Obtain a spectrum license for exclusive use;

- Lease spectrum from existing users (secondary use);

- Use dedicated unlicensed spectrum;

- Use underlay transmission (ultra-wideband);

- Opportunistic access of the idle spectrum through agile radio.

The ultimate advantages of secondary use might be easy to perceive, but its obstacles, hidden costs, and efficiencies are still unclear. The availability of spectrum and the level of participation of primary spectrum users have to be taken into account. A limited pool of usable spectrum can result in insufficient liquidity in the market. Technological factors may place a limit on spectrum sharing capability. Different technologies of primary and secondary users may cause barriers in developing secondary use of spectrum. Equipment capabilities and costs also influence the feasibility of the secondary use. One important assumption we have to make for this framework is the availability of Software Defined Radios (SDR). The capabilities of SDR can be used to provide real-time spectrum management functions that are essential parts of the pre-conditions of the markets. The degree of flexibility a device should have in order to function in the spectrum market also needs to be determined.

Secondary users hold a risky position in their service operations since they may not have direct control over the availability of radio spectrum, quality of service, and coverage expectation. This may make it difficult for the secondary users to control the quality of service they provide to their clients. Strategic behavior of the primary users is also significant concern for the secondary users, especially when liquidity is low. To approach this problem, 
we need to analyze the viability of secondary users with respect to the types of services, transmission technologies, market settings, transaction costs, and requirements and costs of software defined radios needed in providing spectrum sharing for some levels of quality of service. The study of necessary conditions and the impacts of the SDR and cognitive radios to the secondary use of spectrum are, however, beyond the scope of this dissertation.

\subsection{HOW DO PRIMARY AND SECONDARY USERS FIND EACH OTHER?}

This question gives a rise to technical and economic issues. The market is framed in significant measure by the technical features of the spectrum in question. For example, trading in higher frequency bands implies a local spectrum market because the higher frequencies suffer greater attenuation than lower frequencies. Depending on the type of sharing, demand and supply could become very specific in each of the sharing dimensions, such in time, geographical location, frequency, and so forth. This may result in more complicated matching and negotiation mechanisms, especially when the markets progress towards real-time markets.

The trading of secondary use may occur through intermediaries such as bandwidth brokers or distributed market makers or through the process of online-automated spectrum

sharing and trading in a real-time fashion. In general, the mechanisms of searching for a match between the primary and the secondary users largely rely on types of services, access characteristics, and service levels requested by secondary users. The access types could consist of a long-term lease, a scheduled lease, and a short-term lease or spot markets. Each type requires different discovery mechanisms and applies with different levels of service agreements. 


\subsection{WHAT HAS TO BE NEGOTIATED? AND HOW?}

The essence of the problem is to identify technical parameters that primary and secondary users must negotiate for spectrum usage right trading. Hence, the necessity of developing practical negotiation mechanisms becomes apparent. We need to investigate whether the negotiation mechanisms can be generalized. In an ideal case, all systems could apply a uniform negotiation pattern by means of constructing an agreement on standard-based parameters. However, if the spectrum sharing is highly dependent on technologies, the negotiation mechanisms would depend on specific types of technologies or market mechanisms. In both cases, the development of the negotiation mechanisms needs to be more specifically defined.

Negotiation parameters generally include technical (frequency, location, time, transmit power, multiplexing form, and so forth), financial (price and payment options), service quality (interference protection, signal-to-noise ratio, and so on). Actual parameters in negotiation may be more or less specific depending on characteristics of services offered by the primary user and the secondary user.

Similar to the discovery mechanism, the negotiation mechanism depends on the types of spectrum use and access models. The performance of each possible mechanism could be measured by its transaction costs (costs associated with providing information, matching mechanism, negotiation, payment, enforcement, and so forth), ability to support different types of services, and ability to support real-time markets.

It is also important to take into account the situation where a secondary user intends to access radio spectrum on an opportunistic use basis. This type of users must utilize a cognitive radio system that is capable of detecting spectrum environment for opportunity to access spectrum and adapting its transmission to avoid harmful interference to the primary user. In this case, the set of negotiation parameters may be different. The negotiation process may be a one-time process provided that opportunistic users has equipped with proven adaptive techniques or has complied with a set of initial agreements. 


\subsection{AN AGENT-BASED MODEL FOR SECONDARY USE OF RADIO SPECTRUM}

This chapter introduces and provides description of the agent-based model for secondary use of radio spectrum. First, Section 6.1 gives the model overview and description of the modeling toolkit. Next, the governance structures and the incorporation of Transaction Cost Economics into the agent-based model are discussed in Section 6.2. Section 6.3 describes specifications of agents in the spectrum access model which consists of consumer agents and provider agents. Then, Sections 6.4-6.6 explain the behavioral methods of both agents including agent's learning algorithm, opportunistic behavior modeling, and pricing mechanism. Finally, Sections 6.7 and 6.8 discuss agent's operating environment and model stopping criteria, respectively.

\subsection{MODEL OVERVIEW}

This section introduces a discrete-time agent-based economic model for spectrum access. We use the Recursive Porous Agent Simulation Toolkit (Repast) as an agent modeling toolkit [71, 72]. Repast provides a collection of tools that facilitate model development, data collection, and analysis on the collected data. The purpose of using the Repast modeling toolkit aims at increasing the reliability and efficiency of the model, as some of the complex and nonresearch-specific parts have been optimized by the toolkits. These advantages also facilitate the replication of research results.

The model consists of two types of economic agents: spectrum access consumers and spectrum access providers. A spectrum access consumer is a new spectrum user who is 
seeking to obtain spectrum access. The consumer is a potential secondary user. A spectrum access provider is a spectrum license holder. The provider may become a primary user if s/he allows secondary use by leasing portions of spectrum to the consumer. Both types of agents and their operating environments can be customized to reflect different scenarios. For example, the spectrum needs of consumers can be set up to represent short-range or long-range communications according to transmit powers, receiver capabilities, and other requirements, or to reflect some well-defined wireless standards. Several configurations of the spectrum use of providers, such as broadcast networks or cellular networks, are possible by defining, among others, casting and directionality of communications, the range of frequencies, transmitter and receiver locations, transmit power, and receiver requirements. The operating environment can be modified to represent urban or rural settings. Our goal is to explore the emerging behavior among agents when the secondary use of spectrum is introduced as an additional method to obtain spectrum access. More specifically, we focus on the questions of when and why consumers would choose secondary use of spectrum and in what form. This study involves identifying processes or scenarios that leads to outcomes through the repeated interactions of these autonomous agents.

\subsection{TRANSACTION COST ECONOMICS AND SECONDARY USE OF SPECTRUM}

Transaction costs exist because of bounded rationality and the potential for opportunistic behavior of transacting partners, as mentioned earlier in Section 3.1. In order to gain insight into the effects of transaction costs, the model incorporates both behavioral assumptions into its agents.

In the case of bounded rationality, consumer and provider agents are subject to several kinds of limitations. In particular, agents do not have complete information about the environment in which they operate. Each provider does not have access to consumer preferences and their potential opportunistic behaviors. Similarly, each consumer does not

have information about behaviors of providers or other consumers. An agent must observe 
the outcomes of the transactions, learn from the interactions, and adapt its action in the dynamic environment. Agents also have limited processing capacity which is implemented by using learning algorithm with minimal complexity. In particular, we use reinforcement learning algorithm to serve the purpose. The algorithm is described in Section 6.4.

For opportunistic behavior, we focus on the impact of interference (i.e., wireless signal interference) on both consumers and providers. To model this, we set up a probability that provider agents would overstate supply quantities; i.e., lease spectrum that could create interference to consumers in order to generate more revenue. Likewise, consumers also have potential to understate their demand quantities; i.e., specify less spectrum than what they actually need in order to reduce cost. It should be noted that overstating the supply might not always be the best strategy for providers. Depending on price elasticity of consumers and characteristics of the market, providers may instead understate their supply quantities in order to generate a higher market price. Understating the supply quantities, however, will not create interference to consumers. The implementation of opportunism in the agent-based model is further explained in Section 6.5.

In the midst of uncertainty, it is not clear how exactly the trading would take place. We expect that different forms of secondary use could affect the magnitude of transaction costs and the level of uncertainty due to potential interference. Corresponding to TCE, the choices of organizational forms are on the market-hierarchy continuum as in Figure 3.1. In the context of secondary use, the organizational form toward the market side implies the real-time spot spectrum market or sharing formations of similar configuration, whereas the hierarchy side suggests the formations similar to the MVNO.

Specifically, we use degree of control as a common indicator to differentiate multiple forms of economic organization that can be used for organizing spectrum access transactions. Degree of control refers to the ability of the organizational form to contain opportunistic behaviors and to facilitate the compatibility of actions among transacting agents. As suggested by TCE [53], Figure 6.1 illustrates that markets have relatively the lowest degree of control, hybrid forms possess intermediate values, and firms (hierarchy) have relatively the highest degree of control.

Considering opportunistic behaviors and coordination capabilities in terms of their in- 


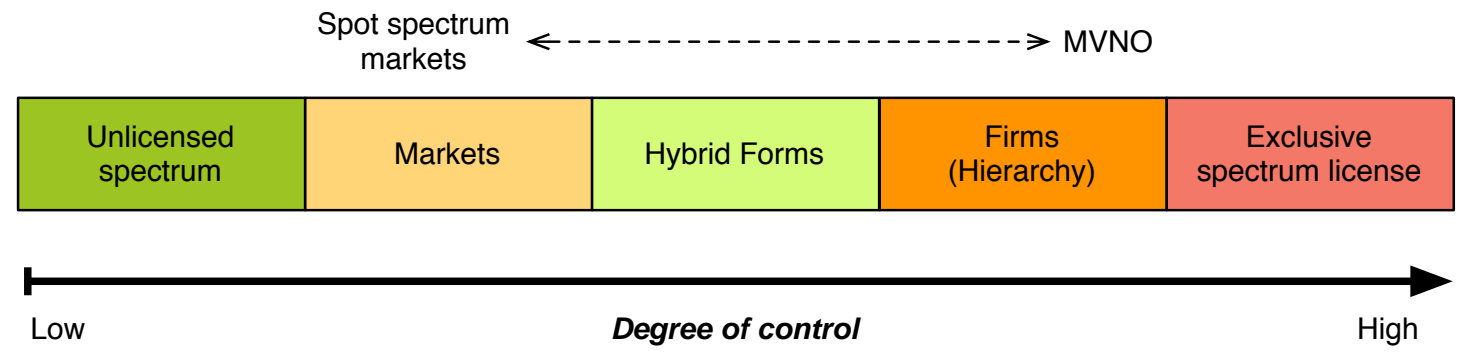

Figure 6.1: Governance structures in secondary use of radio spectrum.

terference effects, using degree of control as an indicator allows us to incorporate traditional methods of spectrum access, namely unlicensed spectrum and exclusive license into the picture. Unlicensed spectrum does not provide interference protection, nor does it facilitate the coordination among unlicensed users. Harmful interferences between unlicensed users or the tragedy of the commons that could render unlicensed spectrum unusable may occur. Therefore, it has lower degree of control compared to markets. On the other hand, a spectrum license gives a licensee an exclusive access and a full interference protection. Thus, a license yields higher degree of control than hierarchy.

\subsection{AGENTS}

Agents are autonomous entities capable of encapsulating their own data and behavioral methods. Agent's data may include its current inventory, price, utility function, and others, while agent's behavioral methods may comprise market protocols, pricing strategies, learning algorithms, and others. It is apparent that some of the methods and data, such as protocols and price, can be publicly accessible to all agents, whereas some of them, such as inventory and strategies, should be contained within an agent. Agents also have communication capability and continuously exchange information with each other to achieve its own goals.

An agent in the spectrum access model is denoted as a spectrum user who utilizes radio spectrum resource to provide wireless services and applications (Section 6.3.1). Spectrum 
users are classified into two subclasses: Consumer agents and provider agents. Consumer agents are spectrum users who are seeking spectrum access. Therefore, they are the potential secondary spectrum users. Provider agents are spectrum users who hold exclusive spectrum licenses and hence they are the potential primary users. Sections 6.3.2 and 6.3.3 present the details of consumer and provider agents, respectively.

\subsubsection{Spectrum Users}

Spectrum users are characterized by their spectrum needs, which in turn are influenced by their application requirements and network configurations. Three main parameters used in the model are frequency bandwidth, duration of access, and utility function. The frequency bandwidth is expressed as a Basic Bandwidth Unit (BBU), the pre-defined amount of bandwidth in $\mathrm{kHz}$. The duration of access is expressed in the number of model time steps.

We adopt a utility-based approach to provide a unified framework for different spectrumbased applications and for spectrum users with different priority levels. Specifically, each spectrum user maintains its utility ${ }^{1}$ function as a perceived value (utility) of the received Quality of Service (QoS) of wireless transmission. We assume that the QoS depends on the received Signal to Interference-plus-Noise Ratio (SINR). We also assume that the data rate is an increasing function of the received SINR. Different users may have different utilities from the same QoS depending on the service and application. Thus, various service requirements can be embedded in each user's utility function.

Applications with hard requirements on SINR such as a voice service require the received SINR above a certain threshold to get an acceptable QoS. Users are indifferent to any further increase in SINR above the threshold. If the received SINR drops below the threshold level, the QoS is unacceptable and the user acquires zero utility. Therefore, their utility functions are modeled as a step function, as in Figure 6.2(a). These applications are inelastic in their demand for SINR. On the other hand, applications such as file transfer and e-mail are considered elastic. They tend to tolerate delay and can take advantage of the low data rate.

\footnotetext{
${ }^{1}$ Utility is an economic term used to express the happiness or satisfaction a person derives from consuming goods and services. It also reflects the willingness of the person to pay for goods or services. In our case, the good or service is the QoS that a spectrum user receives.
} 


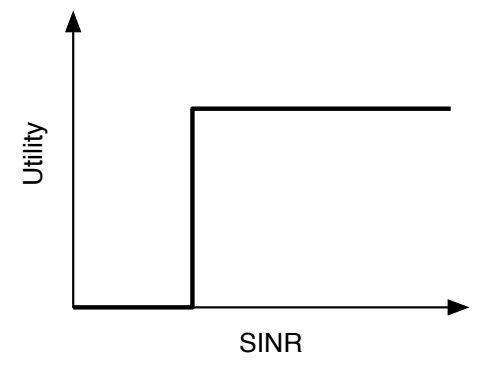

(a)

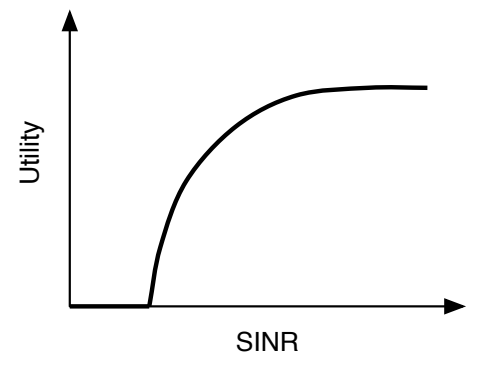

(b)

Figure 6.2: Utility functions: (a) inelastic application and (b) elastic application.

Their utility functions typically are in the form of diminishing returns to scale and take the shape of a concave function, as in Figure 6.2(b) [73, 74, 75].

The communications system of each spectrum user is represented by a set of transmitters and receivers that belong to that user. Obviously, the direction of communications is from the transmitter to the receiver. Casting (e.g., uni-cast, multi-cast, and broadcast) is achieved by defining the pair or connectivity between transmitter(s) and receiver(s). Figure 6.3 illustrates different possible configurations of the spectrum user system.

In summary, the utility-based framework and the configuration of communications system are used to describe each spectrum user. Table 6.1 presents these general parameters of spectrum user.

\subsubsection{Consumer Agents}

Consumer agents are spectrum users who do not currently own exclusive spectrum license and thus are actively seeking spectrum access. They are the potential secondary spectrum users. We use degree of control $(d)$ to identify organizational forms that can carry out spectrum access transactions, as mentioned earlier in Section 6.2. Following our choices of organizational forms to carry out spectrum transactions in Figure 6.1, we can assign values of degree of control so that consumers using the unlicensed spectrum will derive zero degree of control; and those using the exclusive license will acquire degree of control of one, as in 


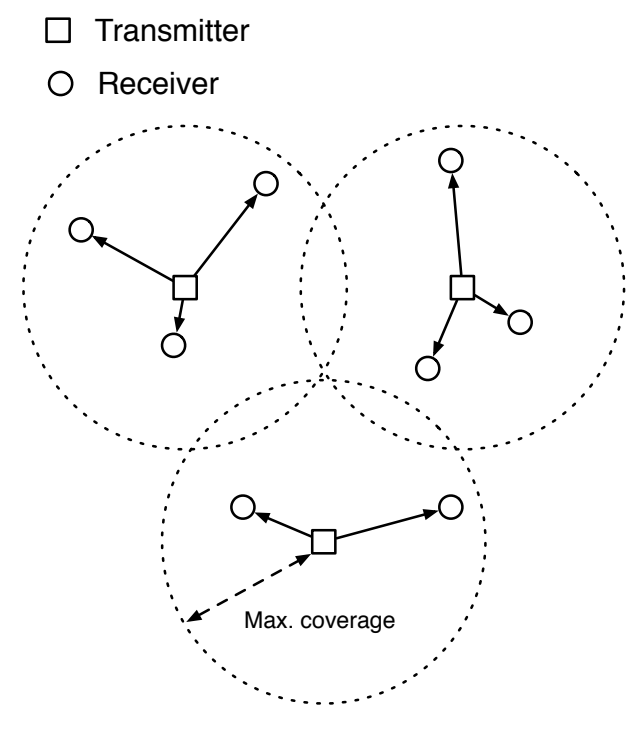

(a)

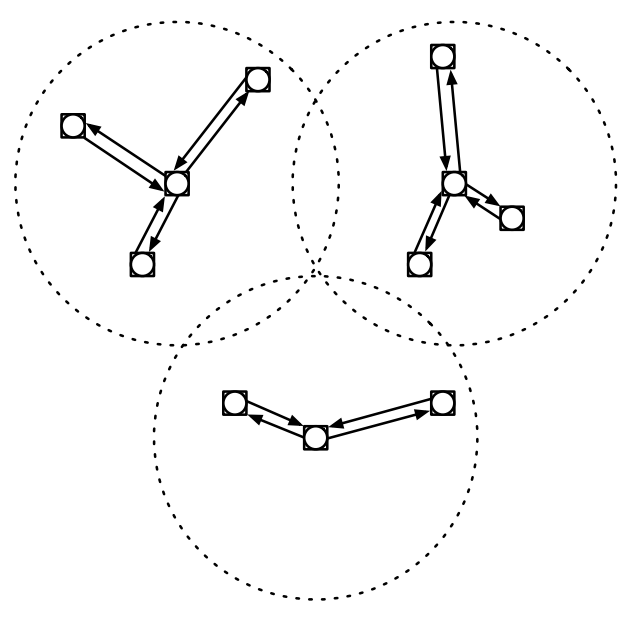

(b)

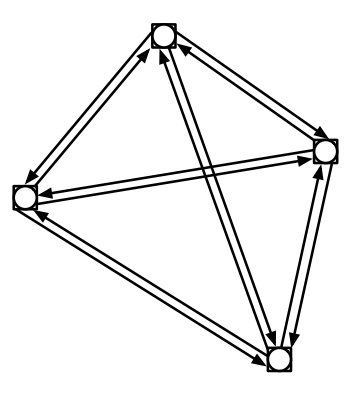

(c)

Figure 6.3: Spectrum user's wireless system: (a) one-way broadcast network, (b) two-way infrastructure network, and (c) ad hoc network. 


\section{Table 6.1: General Parameters of Spectrum Users}

\begin{tabular}{|c|c|}
\hline Symbol & Description \\
\hline$\overline{\bar{i}}$ & Spectrum user index \\
\hline$N_{T, i}$ & Number of transmitters of spectrum user $i$ \\
\hline$N_{R, i}$ & Number of receivers per transmitter of spectrum user $i$ \\
\hline$M_{i}$ & Number of BBUs per transmitter of spectrum user $i$ \\
\hline$D_{\max _{i}}$ & Maximum coverage of spectrum user $i$ 's transmitters in meters \\
\hline$S E N_{i}$ & Receiver sensitivity of spectrum user $i$ in $\mathrm{dBm}$ \\
\hline$R E U_{i}$ & Frequency reuse factor of spectrum user $i$ \\
\hline $\mathcal{U}_{i}(\gamma)$ & Utility function of spectrum user $i$, where $\gamma$ is the received SINR \\
\hline$\gamma_{i}^{*}$ & Spectrum user $i$ 's SINR threshold in $\mathrm{dB}$ \\
\hline$\eta_{i}$ & Degree of concavity of spectrum user $i$ 's utility function \\
\hline$u_{i, t}$ & $\begin{array}{l}\text { Received utility of spectrum user } i \text { at period } t \text { as a perceived value of the } \\
\text { received quality of service (QoS) }\end{array}$ \\
\hline$s_{i, t}$ & Surplus of spectrum user $i$ at period $t$ \\
\hline$c_{i, t}$ & Cost of spectrum access of spectrum user $i$ at period $t$ \\
\hline$\alpha_{u, i}$ & QoS sensitivity of spectrum user $i$ \\
\hline$\alpha_{c, i}$ & Cost sensitivity of spectrum user $i$ \\
\hline
\end{tabular}




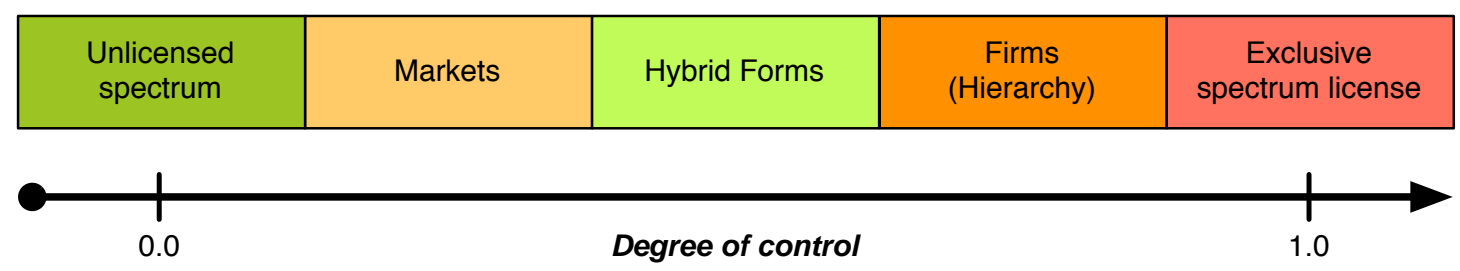

Figure 6.4: Degree of control values of different organizational forms in secondary use of radio spectrum.

Figure 6.4. It should be noted that these degree of control values should be interpreted as an ordinal measure and not by their absolute values, as the objective here is to convey the relative ranking of different organizational forms.

Accordingly, consumer agents have three main methods to obtain spectrum access as follows:

- Unlicensed spectrum: When using unlicensed spectrum $\left(d_{c}=0.0\right)^{2}$, a consumer agent randomly selects an operating frequency inside the unlicensed spectrum band at every model time step. This implementation is similar to the frequency hopping technique currently used in the unlicensed band. It also implies that the consumer agent is free to select other spectrum access options as soon as the current time step ends. The cost of using unlicensed spectrum is zero. The consumer, however, is taking risk of being interfered with by other unlicensed spectrum users.

- Secondary use: If a consumer agent selects secondary use $\left(0.0<d_{c}<1.0\right)$, the $d_{c}$ value will represent the selected degree of control by the consumer. The provider must guarantee the selected degree of control to be eligible for a transaction with this consumer. Therefore, consumer's $d_{c}$ choice can control the outcome of economic organization that will be used to organize the current spectrum access transaction. The choice of $d_{c}$ implies the ability to contain opportunistic behaviors of the provider agents. A higher value of $d_{c}$ provides greater protection against opportunism, as mentioned earlier; it may, however, incur additional cost as the consumer is trying to structure the transaction to reduce

\footnotetext{
${ }^{2} d_{c}$ and $d_{p}$ denote consumer's and provider's choices of degree of control, respectively.
} 
risks of opportunism.

With the chosen value of $d_{c}$, the consumer agent will announce a spectrum access bid at period $t$ that includes the following information:

- Amount of spectrum $\left(M_{c}^{\text {total }}\right)$

- Degree of control $\left(d_{c, t}\right)$

- Access duration $\left(l_{c, t}\right)$

- Bid price $\left(p_{c, t}^{b i d}\right)$

The buying bids from consumer agents and selling offers ${ }^{3}$ from provider agents will enter a competitive bidding process ${ }^{4}$. The outcome will determine the transaction partner (provider), the final secondary use price (i.e., the cost of spectrum access), the operating frequency, and the organizational form $(d)$ that both parties are agreed upon. We assume that the provider can arrange secondary access without interference over the entire simulation area. In other words, we assume that the simulation area represents the unused part of the provider's spectrum. We also assume that consumer pays an additional fixed cost that is linearly increased with $d$ for every secondary use transaction.

- Exclusive license: When selecting an exclusive license $\left(d_{c}=1.0\right)$, consumer pays the license cost $\left(C_{\text {license }}\right)$. The consumer agent is locked in and cannot select other choices of spectrum access until the license is expired. We assume that when the consumer agent exercises the exclusive license option, s/he can expect an interference-free operation over the entire simulation area.

Given an array of choices to obtain spectrum access, each consumer agent explores spectrum access options by repeatedly choosing among several combinations of $\{$ degree of control, access duration $\}$ or $\left\{d_{c}, l_{c}\right\}$ to satisfy his/her spectrum demand. Since consumer agents are also spectrum users, they inherit the general parameters of spectrum users in Table 6.1. Table 6.2 summarizes notations and parameters of consumer agents in addition to those of the spectrum users.

For each spectrum transaction, the received utility $(u)$ is the minimum received utility of all receivers of that consumer. Then, each consumer $j$ calculates a surplus value $\left(s_{j, t}\right), \mathrm{s} / \mathrm{he}$

\footnotetext{
${ }^{3}$ See Section 6.3 .3

${ }^{4}$ See Section 6.6
} 
Table 6.2: Additional Parameters of Consumer Agents

\begin{tabular}{c||l}
\hline Symbol & Description \\
\hline \hline$M_{c_{j}}^{\text {total }}$ & Total number of BBUs required by consumer $j$ \\
$N_{d}$ & Number of degree of control choices for consumers \\
$d_{c_{j}, t}$ & Degree of control of consumer $j$ at period $t$ \\
$\mathcal{D}_{c}$ & Set of consumer's degree of control choices, $\left\{0, \frac{1}{N_{d}-1}, \frac{2}{N_{d}-1}, \ldots, \frac{N_{d}-1}{N_{d}-1}\right\}$ \\
$N_{l}$ & Number of lease durations for consumers \\
$l_{c_{j}, t}$ & Lease duration of consumer $j$ at period $t$ \\
$\mathcal{L}_{c}$ & Set of consumer's lease duration choices in number of model time steps \\
$p_{c_{j}, t}^{b i d}$ & Bid price per BBU of consumer $j$ at period $t$ \\
\hline
\end{tabular}

received as a function of the received utility $\left(u_{j, t}\right)$ and the cost of spectrum access $\left(c_{j, t}\right)$ :

$$
s_{j, t}=\alpha_{u, j} u_{j, t}-\alpha_{c, j} c_{j, t}
$$

The objective of the consumer is commonly assumed to maximize his/her surplus [74, 75]. Accordingly, consumers use a learning algorithm (Section 6.4.1) to adapt their preferences on $d_{c_{j}, t}$ and $l_{c_{j}, t}$ based on the received surplus $\left(s_{j, t}\right)$ from each transaction. The price update of consumer's bid $\left(p_{c_{j}, t}^{b i d}\right)$ is discussed in Section 6.6.

\subsubsection{Provider Agents}

Providers in spectrum access model are spectrum users who currently own exclusive spectrum licenses. Therefore, they are the potential primary spectrum users. Similar to consumer agents, provider agents' spectrum utilization is characterized by their application requirements and network configurations. The operating frequency of transmitters and receivers is within the bounds of provider's spectrum license. The providers can sublease unused parts of 
their spectrum in terms of the number of BBUs and lease durations specified in consumer's spectrum access bids, subjected to availability.

Each provider agent selects a degree of control $\left(d_{p}\right)$, which will represent the minimum threshold that is acceptable for the provider. The consumer must have an adequate degree of control $\left(d_{c} \geq d_{p}\right)$ to be eligible for a transaction with this provider. Therefore, the $d_{p}$ choice controls the outcome of organization that will be used to manage spectrum transaction of this provider. The choice of $d_{p}$ also reflects the ability of the provider to contain opportunistic behaviors of consumers in the transaction. In this case, the high-level $d_{p}$ may limit the number of eligible consumers and, hence, the demand for secondary use of spectrum that the provider may serve.

The range of $d_{p}$ is between 0.0 and 1.0. The minimum value of $d_{p}$ is equal to the minimum value of $d_{c}$ for secondary use. When the provider selects this minimum value, it indicates that $\mathrm{s} /$ he is willing to conduct a transaction with any consumer agent. The maximum value of $d_{p}$ is 1.0 , which gives the provider an option not to participate in secondary use. This implementation is feasible because the maximum value of $d_{c}$ for secondary use is always less than 1.0. With the selected value of $d_{p}$, the provider agent will announce a spectrum access offer at period $t$ that includes the following information:

- Amount of share spectrum $\left(M_{p, t}^{\text {share }}\right)$

- Minimum degree of control $\left(d_{p, t}\right)$

- Maximum lease duration $\left(l_{p, t}\right)$

- Frequency channel $\left(f_{p, t}\right)$

- Ask price $\left(p_{p, t}^{a s k}\right)$

The values of $M_{p, t}^{\text {share }}, l_{p, t}$, and $f_{p, t}$ may vary in each model time step depending on the characteristic of provider's spectrum usage and the ongoing secondary use transactions. The secondary use offers from providers and the bids ${ }^{5}$ from consumers will enter a competitive bidding process ${ }^{6}$. The outcome will determine the transaction partner (consumer), the secondary use price (i.e., revenue from secondary use), and the organizational form $(d)$ of the

\footnotetext{
${ }^{5}$ See Section 6.3.2

${ }^{6}$ See Section 6.6
} 
Table 6.3: Additional Parameters of Provider Agents

\begin{tabular}{c||l}
\hline Symbol & Description \\
\hline \hline$M_{p_{k}, t}^{\text {share }}$ & Number of share BBUs of provider $k$ at period $t$ \\
$d_{p_{k}, t}$ & Degree of control of provider $k$ at period $t$ \\
$\mathcal{D}_{p}$ & Set of provider's degree of control choices, $\left\{\frac{1}{N_{d}-1}, \frac{2}{N_{d}-1}, \ldots, \frac{N_{d}-1}{N_{d}-1}\right\}$ \\
$l_{p_{k}, t}$ & Maximum lease duration of provider $k$ at period $t$ \\
$f_{p_{k}, t}$ & Frequency channel of provider $k$ for secondary use at period $t$ \\
$p_{p_{k}, t}^{a s k}$ & Ask price per BBU of provider $k$ at period $t$ \\
\hline
\end{tabular}

secondary use transaction. These information will be used by the provider to update the availability of spectrum in the subsequent offers.

With an array of degree of control choices, each provider agent repeatedly samples different $d_{p}$ choices to find the best option for the current scenario. Like consumer agents, providers also inherit the general parameters of spectrum users in Table 6.1. Table 6.3 provides additional notations and parameters of provider agents.

For each transaction, the provider calculates the received utility $(u)$, which is the minimum received utility of all of the provider's receivers. Then, each provider $k$ uses the received utility $\left(u_{k, t}\right)$, the exclusive license cost $\left(C_{\text {license }}\right)$, and the secondary use revenue to compute the surplus value $\left(s_{k, t}\right)$ :

$$
s_{k, t}=\alpha_{u, k} u_{k, t}-\alpha_{c, k} C_{\text {license }}+\text { revenue }
$$

Similar to consumer agents, the objective of providers is assumed to maximize his/her surplus $[74,75]$. Accordingly, providers use a learning algorithm (Section 6.4.2) to adapt their preferences on $d_{p_{k}, t}$ based on the received surplus $\left(s_{k, t}\right)$ from each transaction. The price update of provider's offer $\left(p_{p_{k}, t}^{a s k}\right)$ is discussed in Section 6.6. 


\subsection{LEARNING ALGORITHM}

Given the result of the transaction, both consumer and provider agents adapt their future actions in order to maximize their own objectives. One of the popular learning algorithms used to capture the natural learning process of human and organization is reinforcement learning [76, 77]. Unlike other supervised learning approaches, such as pattern recognition and artificial neural networks that learn from examples, reinforcement learning focuses on real-time adaptive interactions with an uncertain environment which is often difficult to obtain examples and the desired responses for all situations.

The underlying concept of reinforcement learning is that the propensity to take any particular action should be increased or reinforced if it produces favorable outcomes and decreased if it leads to negative results [78]. The algorithm is decentralized and only required the past information of agent's actions and the corresponding payoffs. The advantage of reinforcement learning is in its simplicity that does not require high-level processing capabilities from the agent. Therefore, this learning algorithm fulfills the notion of bounded rationality in the transaction cost theory.

In particular, we implement the reinforcement comparison method which establishes a reference level of result (reference reward) and uses this reference value to evaluate the outcome of the future transactions [78]. The reinforcement comparison method maintains the propensity or preference for each action and uses the propensity values to calculate the probability that the particular action will be chosen in the next transaction. Figure 6.5 illustrates the process of the reinforcement comparison method.

\subsubsection{Consumer Learning}

Table 6.4 provides notations and parameters of consumer's learning process. In the case of consumer agents, the action is the selection of $\left\{d_{c}, l_{c}\right\}$. The probability of consumer $j$ selecting any $d_{c}$ and $l_{c}$ at period $t_{1}$ according to the softmax action selection rules is:

$$
\rho_{c_{j}, t_{1}}\left(d_{c}, l_{c}\right)=\frac{\exp \left[q_{c_{j}, t_{1}}\left(d_{c}, l_{c}\right)\right]}{\sum_{a \in \mathcal{D}_{c}, b \in \mathcal{L}_{c}} \exp \left[q_{c_{j}, t_{1}}(a, b)\right]}
$$




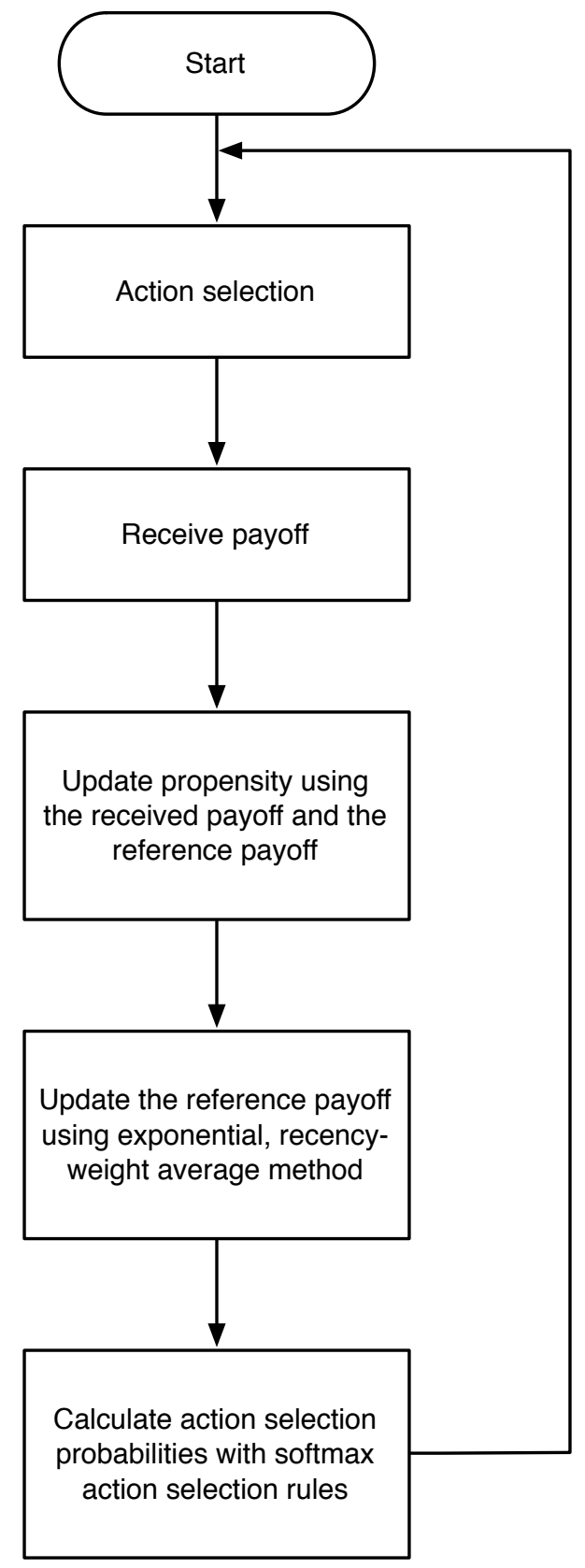

Figure 6.5: Reinforcement comparison learning process. 
Table 6.4: Learning Parameters of Consumer Agents

\begin{tabular}{|c|c|}
\hline Symbol & Description \\
\hline$s_{j, t}^{r}$ & Reference surplus of consumer $j$ at period $t$ \\
\hline$\alpha_{s c}$ & Consumer's step-size parameter for reference surplus update, $0<\alpha_{s c} \leq 1$ \\
\hline$\beta_{s c}$ & $\begin{array}{l}\text { Consumer's step-size parameter for propensity values update based on the } \\
\text { surplus received, } \beta_{s c}>0\end{array}$ \\
\hline$q_{c_{j}, t}\left(d_{c}, l_{c}\right)$ & $\begin{array}{l}\text { Propensity that consumer } j \text { selects a combination of degree of control } d_{c} \\
\text { and lease duration } l_{c} \text { at period } t ; d_{c} \in \mathcal{D}_{c}, l_{c} \in \mathcal{L}_{c}\end{array}$ \\
\hline$\rho_{c_{j}, t}\left(d_{c}, l_{c}\right)$ & $\begin{array}{l}\text { Probability that consumer } j \text { selects a combination of degree of control } d_{c} \\
\text { and lease duration } l_{c} \text { at period } t ; d_{c} \in \mathcal{D}_{c}, l_{c} \in \mathcal{L}_{c}\end{array}$ \\
\hline$\delta_{c_{j}}$ & Probability that consumer $j$ does not follow the learning algorithm \\
\hline
\end{tabular}

The consumer obtains spectrum access based on the values of $\left\{d_{c_{j}, t_{1}}, l_{c_{j}, t_{1}}\right\}$ selected from the probability distribution. Assume that the transaction (i.e., unlicensed, secondary use, or exclusive license access) concludes at period $t_{2}$, the propensity of selecting $d_{c_{j}, t_{1}}$ and $l_{c_{j}, t_{1}}$ is updated by the difference between the received surplus $\left(s_{j, t_{2}}\right)$ and the reference surplus $\left(s_{j, t_{1}}^{r}\right)$ :

$$
q_{c_{j}, t_{2}}\left(d_{c_{j}, t_{1}}, l_{c_{j}, t_{1}}\right)=q_{c_{j}, t_{1}}\left(d_{c_{j}, t_{1}}, l_{c_{j}, t_{1}}\right)+\beta_{s c}\left(s_{j, t_{2}}-s_{j, t_{1}}^{r}\right)
$$

According to this update, the probability of selecting the same action on the next transaction will be increased if the received surplus is higher than the reference value, and decreased if the received surplus is lower. This adjustment is controlled by the step-size parameter $\beta_{s c}$.

After the propensity update in (6.4), the reference surplus is updated to include the received surplus $\left(s_{j, t_{2}}\right)$. Instead of taking an average of all past surpluses as a new reference value, we weight the recent surpluses more heavily than the older ones. This exponential, recency-weighted average method is appropriate for a non-stationary environment [78]. The reference surplus update is as follows:

$$
s_{j, t_{2}}^{r}=s_{j, t_{1}}^{r}+\alpha_{s c}\left(s_{j, t_{2}}-s_{j, t_{1}}^{r}\right)
$$

Here, $\alpha_{s c}$ is the step-size parameter that controls the weight of reference surplus update. 
In addition to this learning process, we want to make sure that consumer agents explore the whole $\left\{d_{c}, l_{c}\right\}$ space and do not bind to certain solutions. We add a stochastic element into the model by assigning a small probability, $\delta_{c_{j}}$, that consumer $j$ will not follow the probability distribution $\rho_{c_{j}, t}\left(d_{c}, l_{c}\right)$ as suggested by the reinforcement learning in (6.3). Instead, consumer $j$ will randomly select $\left\{d_{c}, l_{c}\right\}$ choices (i.e., using the uniform distribution):

$$
\rho_{c_{j}, t}\left(d_{c}, l_{c}\right)= \begin{cases}\frac{\exp \left[q_{c_{j}, t}\left(d_{c}, l_{c}\right)\right]}{\sum_{a \in \mathcal{D}_{c}, b \in \mathcal{L}_{c}} \exp \left[q_{c_{j}, t}(a, b)\right]} & \text { with probability } 1-\delta_{c_{j}} \\ \frac{1}{N_{l}\left(N_{d}-2\right)} & \text { with probability } \delta_{c_{j}}\end{cases}
$$

\section{Example of the Learning Process}

To demonstrate the learning process of spectrum access model, Figure 6.6 shows the process of one consumer agent. In this example, there is only one consumer operating in the environment. Obviously, the best strategy for this consumer is to use unlicensed spectrum because there is no cost to acquire spectrum access and there is no possibility of interference. The graph shows that the consumer tries out different combinations of degrees of control and lease durations (i.e., the duration s/he remains on a particular degree of control), and finally converges to 0.0 (unlicensed spectrum) as a choice of spectrum access.

Another example demonstrates the surplus-maximizing objective of consumer agents. Figure 6.7 shows an average surplus of ten consumer agents operating in the same environment. The graph shows that the average surplus fluctuates over time due to the consumer's experiments with various $\left\{d_{c}, l_{c}\right\}$ combinations, in which the current tryout may produce a lower or higher surplus than the previous one. The reinforcement learning, however, allows each consumer to learn and adapt his/her selection to maximize his/her objective (i.e., surplus). As a result, we observe that the average surplus increases over time.

\subsubsection{Provider Learning}

The learning process of provider agents is the selection of degree of control value $\left(d_{p}\right)$ to control the organization of spectrum access transaction. Table 6.5 summarizes notation and 


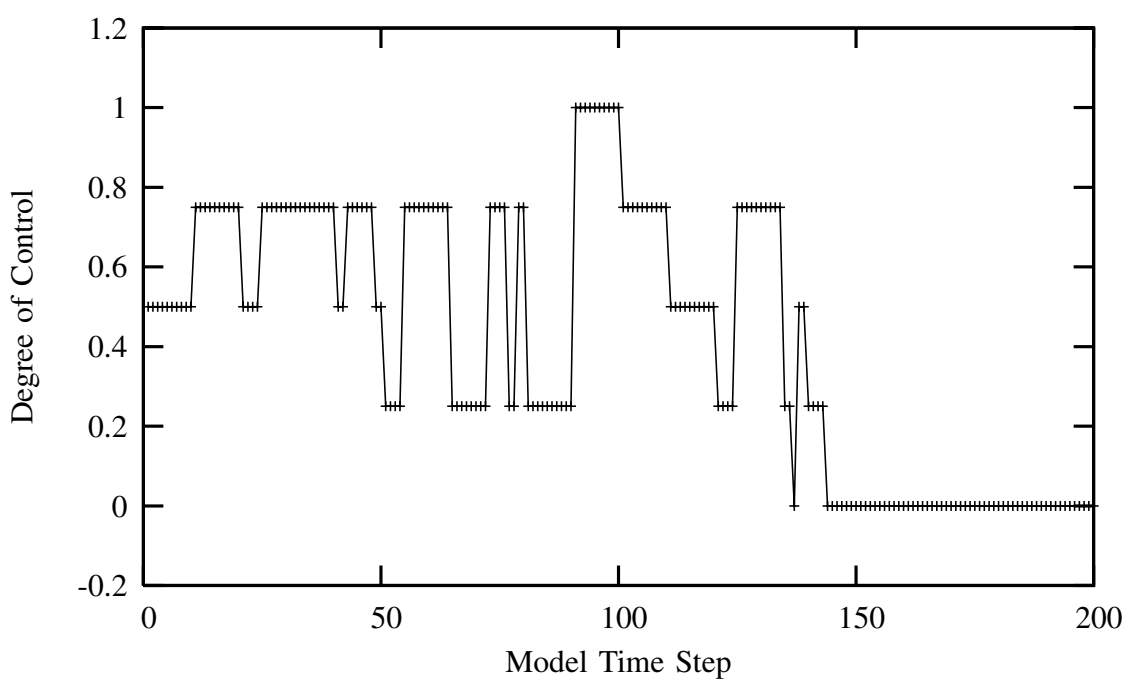

Figure 6.6: Learning process of one consumer agent.

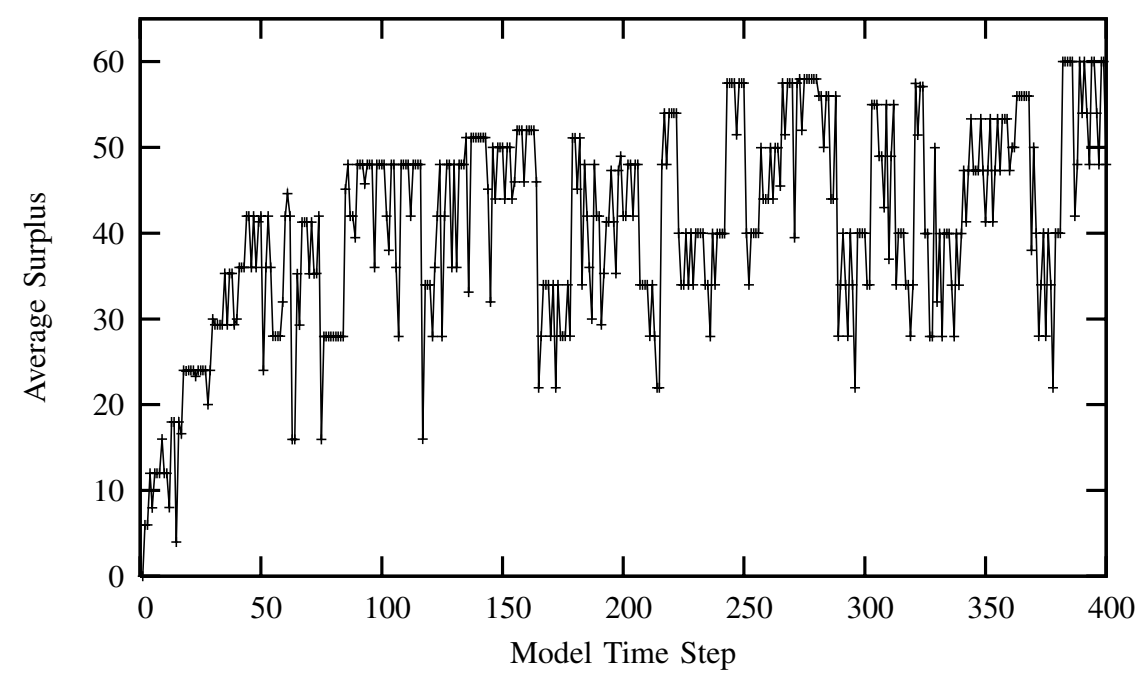

Figure 6.7: Average surplus of ten consumer agents. 
Table 6.5: Learning Parameters of Provider Agents

\begin{tabular}{c||l}
\hline Symbol & Description \\
\hline \hline$s_{k, t}^{r}$ & Reference surplus of provider $k$ at period $t$ \\
$\alpha_{s p}$ & Provider's step-size parameter for reference profit update, $0<\alpha_{s p} \leq 1$ \\
$\beta_{s p}$ & $\begin{array}{l}\text { Provider's step-size parameter for propensity values update based on the } \\
\text { surplus received, } \beta_{s p}>0\end{array}$ \\
$q_{p_{k}, t}\left(d_{p}\right)$ & $\begin{array}{l}\text { Propensity that provider } k \text { selects a degree of control } d_{p} \text { at period } t ; d_{p} \in \mathcal{D}_{p} \\
\rho_{p_{k}, t}\left(d_{p}\right)\end{array}$ \\
$\delta_{p_{k}}$ & $\begin{array}{l}\text { Probability that provider } k \text { selects a degree of control } d_{p} \text { at period } t ; d_{p} \in \mathcal{D}_{p} \\
\text { Probability that provider } k \text { does not follow the learning algorithm }\end{array}$ \\
\hline
\end{tabular}

parameters of provider's learning. The process follows the reinforcement comparison method. The probability of provider $k$ selecting any $d_{p}$ at period $t_{1}$ is:

$$
\rho_{p_{k}, t_{1}}\left(d_{p}\right)=\frac{\exp \left[q_{p_{k}, t_{1}}\left(d_{p}\right)\right]}{\sum_{a \in \mathcal{D}_{p}} \exp \left[q_{p_{k}, t_{1}}(a)\right]}
$$

Assume that the transaction that starts at period $t_{1}$ concludes at period $t_{2}{ }^{7}$, the propensity of selecting $d_{p_{k}, t_{1}}$ is updated by the difference between the received surplus $\left(s_{k, t_{2}}\right)$ and the reference surplus $\left(s_{k, t_{1}}^{r}\right)$ :

$$
q_{p_{k}, t_{2}}\left(d_{p_{k}, t_{1}}\right)=q_{p_{k}, t_{1}}\left(d_{p_{k}, t_{1}}\right)+\beta_{s p}\left(s_{k, t_{2}}-s_{k, t_{1}}^{r}\right)
$$

where $\beta_{s p}$ controls the rate of propensity update. Then, the reference surplus is updated as follows:

$$
s_{k, t_{2}}^{r}=s_{k, t_{1}}^{r}+\alpha_{s p}\left(s_{k, t_{2}}-s_{k, t_{1}}^{r}\right)
$$

where $\alpha_{s p}$ controls the weight of reference value update.

We also include a stochastic element into the model to make sure that providers explore the whole $\left\{d_{p}\right\}$ space and do not bind to certain solutions. Here, we assign a small probability,

\footnotetext{
${ }^{7}$ Each provider may be involved in many spectrum transactions at a time. The $d_{p}$ update is on a pertransaction basis.
} 
$\delta_{p_{k}}$, that provider $k$ will not follow the probability distribution $\rho_{p_{k}, t}\left(d_{p}\right)$ as suggested by the reinforcement learning in (6.7). The provider $k$ will, instead, randomly select $\left\{d_{p}\right\}$ choices using the uniform distribution:

$$
\rho_{p_{k}, t}\left(d_{p}\right)= \begin{cases}\frac{\exp \left[q_{p_{k}, t}\left(d_{p}\right)\right]}{\sum_{a \in \mathcal{D}_{p}} \exp \left[q_{p_{k}, t}(a)\right]} & \text { with probability } 1-\delta_{p_{k}} \\ \frac{1}{N_{d}-1} & \text { with probability } \delta_{p_{k}}\end{cases}
$$

\subsection{OPPORTUNISTIC BEHAVIOR MODELING}

As mentioned earlier in Section 3.1, transaction costs emerge because transacting partners have a potential to behave opportunistically in order to take advantages in a trade. In this section, we incorporate the potential opportunistic behavior into agents in the model.

\subsubsection{Consumer Opportunistic Behavior}

Recall from Section 6.2, we focus on the impact of interference as a result of opportunistic transactions. An opportunistic consumer will understate the original demand quantity in order to reduce secondary use cost. When announcing a spectrum access bid, the original quantity $M_{c}^{\text {total }}$ will be replaced with the new quantity, $M_{c}^{\text {total }}{ }^{*}$. The opportunistic consumer will operate in provider's spectrum in excess of the quantity stated in the bid. Hence, the effect of consumer's opportunism is the potential interference to the provider's receivers. To simplify the model, we use $M_{c}^{\text {total }}{ }^{*}=M_{c}^{\text {total }} / 2$ for all opportunistic consumer agents ${ }^{8}$. Table 6.6 presents the parameters of consumer's opportunistic behavior modeling.

Opportunistic consumers can also learn from their opportunistic behaviors in the current transaction and decide whether they should act opportunistically in the future transactions. We use reinforcement comparison method for this learning process. Let the opportunistic

\footnotetext{
${ }^{8}$ Although we expect that opportunistic spectrum users would abuse the transaction at a small margin (e.g., at 10\%), we select an aggressive margin to intensify the effects of opportunistic behavior in order to speed up the convergence of the agent-based model.
} 
Table 6.6: Parameters of Consumer's Opportunistic Behavior

\begin{tabular}{|c|c|}
\hline Symbol & Description \\
\hline 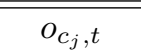 & "Opportunistic choice of consumer $j$ at period $t, o_{c_{j}, t} \in\{0,1\}$ \\
\hline$\pi_{j, t}$ & Profit from opportunistic behavior of consumer $j$ at period $t$ \\
\hline$\pi_{j, t}^{r}$ & Reference profit from opportunistic behavior of consumer $j$ at period $t$ \\
\hline$\alpha_{o c}$ & Consumer's step-size parameter for reference profit update, $0<\alpha_{o c} \leq 1$ \\
\hline$\beta_{o c}$ & $\begin{array}{l}\text { Consumer's step-size parameter for propensity values update based on the } \\
\text { profit received, } \beta_{o c}>0\end{array}$ \\
\hline$q_{c_{j}, t}\left(o_{c}\right)$ & $\begin{array}{l}\text { Propensity that consumer } j \text { chooses an opportunistic behavior } o_{c} \text { at period } \\
t ; o_{c} \in\{0,1\}\end{array}$ \\
\hline$\rho_{c_{j}, t}\left(o_{c}\right)$ & $\begin{array}{l}\text { Probability that consumer } j \text { chooses an opportunistic behavior } o_{c} \text { at period } \\
t ; o_{c} \in\{0,1\}\end{array}$ \\
\hline$T_{\text {base }}$ & Base-level of trust \\
\hline$T_{F}$ & Trust development rate \\
\hline$T_{\text {thres }}$ & Minimum trust threshold to be considered trustworthy \\
\hline$T_{\text {prob }}$ & Probability of participating in a transaction with an untrustworthy partner \\
\hline
\end{tabular}


choice of consumer $j, o_{c_{j}, t}$, be 0 when consumer $j$ decides not to become an opportunist and 1 otherwise. The probability that consumer $j$ will act opportunistically at period $t_{1}$ is:

$$
\rho_{c_{j}, t_{1}}(1)=\frac{\exp \left[q_{c_{j}, t_{1}}(1)\right]}{\exp \left[q_{c_{j}, t_{1}}(0)\right]+\exp \left[q_{c_{j}, t_{1}}(1)\right]}
$$

Assume that the transaction concludes at period $t_{2}$, the propensity of choosing opportunistic choice $o_{c_{j}, t_{1}}$ at period $t_{1}$ is update by the difference between the received profit $\left(\pi_{j, t_{2}}\right)$ and the reference profit $\left(\pi_{j, t_{1}}^{r}\right)$ :

$$
q_{c_{j}, t_{2}}\left(o_{c_{j}, t_{1}}\right)=q_{c_{j}, t_{1}}\left(o_{c_{j}, t_{1}}\right)+\beta_{o c}\left(\pi_{j, t_{2}}-\pi_{j, t_{1}}^{r}\right)
$$

where $\beta_{o c}$ is the step-size parameter that controls the rate of propensity update. Then, the exponential, recency-weighted average method is applied on the reference profit update:

$$
\pi_{j, t_{2}}^{r}=\pi_{j, t_{1}}^{r}+\alpha_{o c}\left(\pi_{j, t_{2}}-\pi_{j, t_{1}}^{r}\right)
$$

where $\alpha_{o c}$ controls the update weight.

The profit from opportunistic behavior can be specified as follows:

- If consumer $j$ does not act opportunistically (i.e., $o_{c_{j}, t_{1}}=0$ ), there will be no gain from this action. Thus, the received opportunistic profit upon the ending of transaction is 0.0 $\left(\pi_{j, t_{2}}=0.0\right)$.

- If consumer $j$ acts opportunistically (i.e., $o_{c_{j}, t_{1}}=1$ ), the profit of this action will be the reduced cost of spectrum access transaction. Thus, the received profit upon the ending of transaction, $\pi_{j, t_{2}}$, is set to the difference between the spectrum access cost with quantity $M_{c}^{\text {total }}$ and the spectrum access cost with quantity $M_{c}^{\text {total }}{ }^{*}$.

- If consumer $j$ receives an interference complaint ${ }^{9}$ from his/her transacting provider, it implies that the transacting provider is suffering interference from one or more secondary use transactions. This situation will obviously discourage the provider from participating in secondary use of spectrum access in the future. As a consequence, consumer $j$ may have to rely on an exclusive license for spectrum access due to the limited availability of spectrum for secondary use. Therefore, upon receiving the interference complaint, consumer $j$ will update the choice of acting opportunistically (i.e., $o_{c_{j}, t_{1}}=1$ ) with the received profit, $\pi_{j, t_{2}}$, of negative exclusive license cost $\left(-C_{\text {license }}\right)$.

\footnotetext{
${ }^{9}$ See Section 6.5.2.
} 


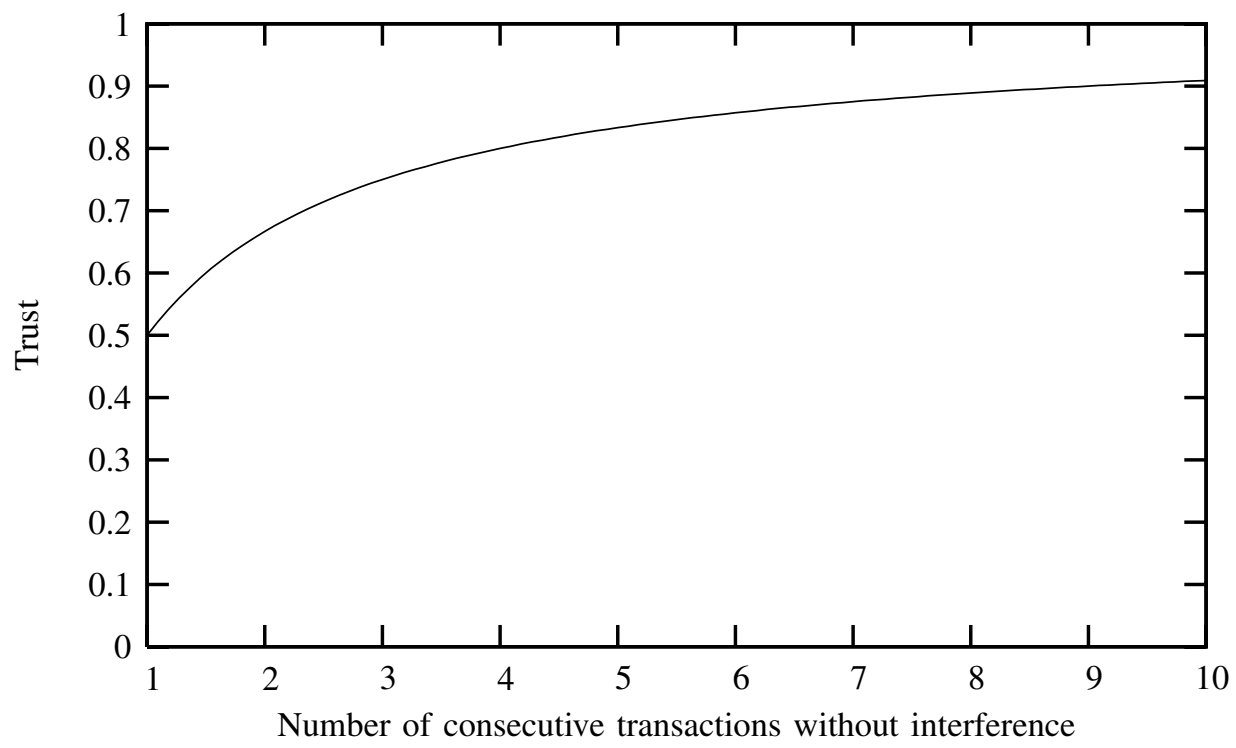

Figure 6.8: Trust development $\left(T_{\text {base }}=0.5\right.$ and $\left.T_{F}=0.5\right)$.

The remaining of this section describes consumer's reaction to the opportunistic behavior of provider agents. We assume that a consumer agent can distinguish his/her transacting partners and develop as well as maintain basic trust information on each of his/her partners. Following Klos's agent-based modeling of trust [79], trust is defined as the ability to act according to expectation. In our case, consumer agents expect secondary use of spectrum without interference. We further assume that the consumer's trust in a particular provider increases with the number of consecutive transactions without interference when the consumer transacts with that provider. The development of consumer's trust is specified by the following equation [79]:

$$
\text { trust }=T_{\text {base }}+\left(1-T_{\text {base }}\right)\left(1-\frac{1}{x T_{F}+1-T_{F}}\right)
$$

where $T_{\text {base }}$ is the base-level of trust; $x$ is the number of consecutive transactions without interference; and $T_{F}$ is a parameter that controls trust development rate. From (6.14), the range of trust value is $[0,1]$. Figure 6.8 illustrates the improvement of trust with the number of transactions without interference. 
Table 6.7: Parameters of Provider's Opportunistic Behavior

\begin{tabular}{|c|c|}
\hline Symbol & Description \\
\hline$o_{p_{k}, t}$ & Opportunistic choice of provider $k$ at period $t, o_{p_{k}, t} \in\{0,1\}$ \\
\hline$\pi_{k, t}$ & Profit from opportunistic behavior of provider $k$ at period $t$ \\
\hline$\pi_{k, t}^{r}$ & Reference profit from opportunistic behavior of provider $k$ at period $t$ \\
\hline$\alpha_{o p}$ & Provider's step-size parameter for reference profit update, $0<\alpha_{o p} \leq 1$ \\
\hline$\beta_{o p}$ & $\begin{array}{l}\text { Provider's step-size parameter for propensity values update based on the } \\
\text { profit received, } \beta_{o p}>0\end{array}$ \\
\hline$q_{p_{k}, t}\left(o_{p}\right)$ & $\begin{array}{l}\text { Propensity that provider } k \text { chooses an opportunistic behavior } o_{p} \text { at period } t \text {; } \\
o_{p} \in\{0,1\}\end{array}$ \\
\hline$\rho_{p_{k}, t}\left(o_{p}\right)$ & $\begin{array}{l}\text { Probability that provider } k \text { chooses an opportunistic behavior } o_{p} \text { at period } \\
t ; o_{p} \in\{0,1\}\end{array}$ \\
\hline
\end{tabular}

We apply the following rules to the trust modeling: When consumer $j$ experiences interferences while using spectrum of provider $k$, consumer $j$ 's trust in provider $k$ is reduced by half. If trust drops below $T_{\text {thres }}$, consumer $j$ will transact with provider $k$ with a probability $T_{\text {prob }}$; otherwise, consumer $j$ will always transact with provider $k$. If consumer $j$ does not experience interferences while transacting with provider $k$, then the trust in provider $k$ starts to increase at the rate shown in Figure 6.8.

\subsubsection{Provider Opportunistic Behavior}

An opportunistic provider will overstate the original supply quantity in order to generate more revenue. In doing this, the opportunistic provider will continue to announce a spectrum access offer even after all spectrum for secondary use has been leased to other consumers. The consumer who transacts with this provider will operate in the frequencies that are being used by other consumer agents. Therefore, the effect of provider's opportunism is the potential interference to the consumer's receivers.

Table 6.7 summarizes the parameters of provider's opportunistic behavior modeling. 
Opportunistic providers use reinforcement comparison to learn and maximize the profit from opportunistic behavior choices. Let the opportunistic choice of provider $k, o_{p_{k}, t}$, be 0 when provider $k$ decides not to become and opportunist and 1 otherwise. The probability that provider $j$ will act opportunistically at period $t_{1}$ is:

$$
\rho_{p_{k}, t_{1}}(1)=\frac{\exp \left[q_{p_{k}, t_{1}}(1)\right]}{\exp \left[q_{p_{k}, t_{1}}(0)\right]+\exp \left[q_{p_{k}, t_{1}}(1)\right]}
$$

Assume that the transaction concludes at period $t_{2}$, the propensity of choosing opportunistic choice $\left(o_{p_{k}, t_{1}}\right)$ at period $t_{1}$ is update by the difference between the received profit $\left(\pi_{k, t_{2}}\right)$ and the reference profit $\left(\pi_{k, t_{1}}^{r}\right)$ :

$$
q_{p_{k}, t_{2}}\left(o_{p_{k}, t_{1}}\right)=q_{p_{k}, t_{1}}\left(o_{p_{k}, t_{1}}\right)+\beta_{o p}\left(\pi_{k, t_{2}}-\pi_{k, t_{1}}^{r}\right)
$$

where $\beta_{o p}$ controls the rate of propensity update. Then, the reference profit is updated as follows:

$$
\pi_{k, t_{2}}^{r}=\pi_{k, t_{1}}^{r}+\alpha_{o p}\left(\pi_{k, t_{2}}-\pi_{k, t_{1}}^{r}\right)
$$

where $\alpha_{o p}$ controls the weight of reference value update.

The profit from provider's opportunistic behavior is described as follows:

- If provider $k$ does not act opportunistically (i.e., $o_{p_{k}, t_{1}}=0$ ), there will be no gain from this action. Thus, the received opportunistic profit upon the ending of transaction is 0.0 $\left(\pi_{k, t_{2}}=0.0\right)$.

- If provider $k$ acts opportunistically (i.e., $o_{p_{k}, t_{1}}=1$ ), the profit of this action will be an additional revenue from spectrum access offers in excess of the provider's truthful capacity. Thus, the received profit upon the ending of transaction, $\pi_{k, t_{2}}$, is the revenue of that opportunistic transaction.

- Provider k's spectrum offers may be rejected by a particular consumer. This implies that the consumer's trust on provider $k$ is below $T_{\text {thres }}$ because the consumer has encountered interference when transacted with provider $k$. With the low trust value, the consumer will avoid making secondary use transactions with provider $k$. As a result, provider $k$ will receive less transactions and, hence, less secondary use revenue. Therefore, upon receiving consumer's rejection of the spectrum offer, provider $k$ will update the choice 
of acting opportunistically (i.e., $o_{p_{k}, t_{1}}$ ) with the received profit, $\pi_{k, t_{2}}$, equivalent to the negative revenue had the offer been transacted (i.e., the opportunity cost).

Next, we discuss provider's reaction to the opportunistic behavior of consumer agents. Providers expect an interference-free operation when they are sharing spectrum for secondary use. In contrast to the consumer's opportunistic modeling where consumers maintain trust information on his/her partners, providers do not implement the trust model. The rationale behind this is based on the real-world implementation. In wireless environment, it is considered difficult and costly for providers to identify the sources of interference (i.e., opportunistic consumers), especially when each provider can serve multiple spectrum access consumers at the same time. Instead, provider $k$ will issue an interference complaint to all consumers who are operating in the provider $k$ 's spectrum as soon as s/he experiences interference while allowing secondary use to these consumers. In addition to this, provider $k$ will exercise the choice of minimum degree of control, $d_{p}$, to limit consumer's opportunistic behavior.

\subsection{SPECTRUM LEASING AND PRICING}

We use an auction to determine the prices of spectrum access transactions between consumer and provider agents. An auction is an institution in which traders exchange messages that include a bid (offer to buy) and an ask (offer to sell). The process gives higher priority in a transaction to higher bids and lower asks.

One of the most celebrated auction mechanisms is a continuous double auction (CDA), which is the dominant institution for real-world equities and derivatives trading. In CDA, bids and asks are publicly announced and traded at any time during the trading period without relying on a central auctioneer [80]. Experiments also indicate that the CDA mechanism can produce reliable price convergence close to theoretical equilibria [81].

Several studies examine the agent-based implementation of CDA [82, 83, 84, 85, 86]. They demonstrate that simple adaptive agents can learn to negotiate and trade at equilibrium price in an environment where many buyers and sellers are simultaneously negotiating transactions. In our agent-based model, we follow a persistent shout double auction algo- 
rithm by Preist and Van Tol $[85,86]$. The auction algorithm is an extension of Gode and Sunder's zero-intelligence (ZI) traders [82] and Cliff's zero-intelligence-plus (ZIP) traders [83]. In a persistent shout double auction, an agent may make an offer to buy or offer to sell at any time. The offer is, however, persistent until the owner revises or removes it in response to other trading activities. Once bids and asks are met, they are removed and a trade takes place.

Our objective of using CDA is to determine the equilibrium price of secondary use transactions given the current supply and demand for spectrum access. Although the negotiation in CDA may result in trades taking place at different prices and away from the equilibrium, several repetitions of auction with the same bids and asks can converge to the equilibrium price $[86,81]$. Therefore, consumer and provider agents in the model will engage in a series of mock auctions before the real auction takes place in the final round. These mock auctions will allow agents to response to market conditions and other trading activities before holding the final auction. Hence, the final auction will result in trades taking place closer to the equilibrium than a single auction [86].

In this iterated auction, a double auction is organized into a number of trading periods (mock auctions). Each trading period consists of multiple discrete time steps called trading rounds. According to Preist and Van Tol $[85,86]$, consumer and provider agents who want to participate in an auction must submit secondary use bids and asks, respectively, in the first trading round. Agents may also choose to update their bids/asks in any of the subsequent trading rounds, otherwise their current bids/asks will stand. A trade takes place when the highest bid is greater than or equal to the lowest ask, and the trade is made at the average of the two prices.

Each agent has its own reservation price (limit price), which is the maximum price a consumer is willing to pay for spectrum access or the minimum price a seller is willing to provide secondary use of spectrum. Reservation prices may vary among agents according to their desire for spectrum access and additional revenue, which are specified by their surplus functions. In the secondary use model, reservation price of consumer agent is calculated such that it produces the same surplus value as using an exclusive license, whereas a provider agent uses an amortized exclusive license cost as its reservation price. Both consumers and 
providers continue to trade until there is no bid or ask left, or their bids and asks have reached the reservation price, in which case trade can no longer occur. These conditions mark the end of a trading period. Once a trading period is over, a new trading period begins with all agents submit the same bids and asks in the first trading round, except using the price information that they have learned from the previous trading period.

From Sections 6.3.2 and 6.3.3, the agent-based model for secondary use operates on a set of conditions that includes degree of control, lease duration, and amount of spectrum. These factors create constraints on secondary use transactions in addition to the reservation price. Let $R, D, L$, and $M$ denote reservation price, degree of control, lease duration, and amount of spectrum, respectively. Also, let $b_{i}$ and $a_{j}$ represent bid $i$ and ask $j$, respectively. To determine the eligibility of consumer's bids and provider's asks, the following conditions are inspected:

- Reserve Price: $R_{b_{i}} \geq \min _{j}\left(R_{a_{j}}\right)$ and $R_{a_{j}} \leq \max _{i}\left(R_{b_{i}}\right)$

- Degree of control: $D_{b_{i}} \geq \min _{j}\left(D_{a_{j}}\right)$ and $D_{a_{j}} \geq \min _{i}\left(D_{b_{i}}\right)$

- Lease duration: $L_{b_{i}} \leq \max _{j}\left(L_{a_{j}}\right)$ and $L_{a_{j}} \geq \min _{i}\left(L_{b_{i}}\right)$

- Amount of spectrum: $M_{b_{i}} \leq \max _{j}\left(M_{a_{j}}\right)$ and $M_{a_{j}} \geq \min _{i}\left(M_{b_{i}}\right)$

Each bid must have a least one ask that satisfies all four constraints to be considered eligible and vice versa. At the beginning and during the trading period, if an update alters any of the four factors or any bid or ask has been removed, the bid-ask eligibility will be rechecked. All ineligible bids and asks are immediately removed.

In addition to the four constraints, a consumer agent may reject to transact with a particular provider agent according to the trust modeling in Section 6.5. This implies that the consumer's trust on a certain provider is dropped below the trust threshold $\left(T_{\text {thres }}\right)$ because of interference. Thus, if the transaction between these two agents is bound to take place in this case, it will occur with a probability $T_{\text {prob }}$, otherwise the provider's ask will be removed.

Agent's algorithm in the auction process has minimal complexity. It consists of simple heuristics and uses simple learning rules. Each agent $i$ maintains a profit margin, $\mu_{i}$, which govern the buying/selling price relative to agent's reservation price, $R_{i}$. Individual agent 
calculates the current price, $p_{i}$, as follows:

$$
p_{i}=R_{i}\left(1+\mu_{i}\right)
$$

For a provider agent, the price $p_{i}$ represents the minimum price at which the provider will make a trade. The profit margin for providers must lies in $[0, \infty)$. This implies that a provider's margin can be raised by increasing $\mu_{i}$ and lowered by decreasing $\mu_{i}$. In the case of a consumer agent, the price $p_{i}$ represents the maximum price at which the consumer is willing to pay. The profit margin for consumers is in the range $[-1,0]$. Therefore, a consumer's profit margin can be increased by decreasing $\mu_{i}$ and reduced by increasing $\mu_{i}$.

Each agent initially assigns a random profit margin. The profit margin $\mu_{i}$ is then adjusted dynamically in response to the actions of other agents and the trading activities. If the agent sets the profit margin too low, it will not make as much profit as it should. On the other hand, if the profit margin is too high, it may be undercut by other agents and will not be able to secure a deal. The decision to increase or decrease the profit margin is based on an objective of maintaining the competitive bids/asks price compared to other agents. In order to do this, an agent maintains a target price, $\tau_{i}\left(\tau_{i}=R_{i}\left(1+\mu_{i}\right)\right)$, and follows the simple heuristics: If trades are not taking place in the current trading period, an agent should set its target to become the most competitive bid/ask. Thus, the target price should be slightly better than its competition. If, however, trades are taking place, an agent should set its target to be slightly better (i.e., higher profit) than the best price that can obtain a transaction. Here, the agent anticipates that it could have asked even a higher profit and still secured a deal.

Let $\tau_{i}$ denotes a target price, $b_{\max }$ represents the highest bid, $a_{\min }$ the lowest ask in the current trading round, and $r$ is a small random variable. The following pseudocode summarizes the heuristic for consumer agents.

For consumer agents:

$$
\begin{aligned}
& \text { if } a_{\min }>b_{\max } \text { then } \\
& \qquad \tau_{i}=(1+r) b_{\max } \\
& \text { else } \\
& \qquad \tau_{i}=(1-r) a_{\min }
\end{aligned}
$$




\section{end if}

The heuristic for provider agents is as follows:

For provider agents:

$$
\text { if } \begin{gathered}
a_{\min }>b_{\max } \text { then } \\
\tau_{i}=(1-r) a_{\min }
\end{gathered}
$$

else

$$
\tau_{i}=(1+r) b_{\max }
$$

\section{end if}

Following Preist and Van Tol [85, 86], we implement $r$ as a random variable uniformly distributed over the range $[0,0.2]$.

Agents who are not participating in an auction (i.e., they are successfully engaged in a trade or they do not wish to participate at the current period) also continuously observe the activities of other agents. In this case, they have little or no incentive to lower their profit margins. However, if the trade activities suggest that they could benefit from raising the profit margin, they should do so. Thus, both active and inactive agents can increase their profit margins, but only active agents can reduce their margin in response to the current condition.

With the target price $\tau_{i}$, an agent adjusts its current price $p_{i}$ towards the target using the Widrow-Hoff with momentum learning rule, which is the adaptation algorithm used in backpropagation in neural networks [87]. The algorithm specifies two parameters: The learning rate coefficient $\beta$ and the momentum $\gamma$. Given the price $p_{i}(t)$ at time $t$, the price at time $t+1$ is given by:

$$
p_{i}(t+1)=\gamma p_{i}(t)+(1-\gamma) \beta\left(\tau_{i}(t)-p_{i}(t)\right)
$$

Here, the price will moves towards the target with the speed determined by $\beta$. The momentum $\gamma$ is used to reduces oscillation in the price adjustment. In our experiments, the values of $\beta$ and $\gamma$ are set to 0.3 and 0.05 , respectively [85, 86].

As mentioned earlier, consumer and provider agents will participate in a series of trading periods (i.e., mock auctions) and the real auction will take place in the final period, in which the price has stabilized. We fix the number of mock auctions to 500 auctions. Figure 6.9 




Figure 6.9: Trade Price vs. Trading Period $\left(N_{C}=13\right.$ and $\left.N_{P}=2\right)$.

demonstrates the price adjustment and trade activities in a series of mock auctions.

\subsection{OPERATING ENVIRONMENT}

Consumer and provider agents reside in an operating environment that is described by a set

of parameters in Table 6.8. These parameters can be customized to create different operating scenarios in addition to the parameters of consumer and provider agents.

Consider communications between a transmitter and a receiver, we assume that all agents use omni-directional antennas. Path loss is assumed to satisfy a log-distance path loss model:

$$
\begin{aligned}
P_{0} & =G_{t} G_{r}\left(\frac{\lambda}{4 \pi d_{r e f}}\right)^{2} P_{t} \\
P_{r} & =P_{0} d^{-\alpha_{p l}}
\end{aligned}
$$


Table 6.8: Parameters of the Operating Environment

\begin{tabular}{c||l}
\hline Symbol & Description \\
\hline \hline$N_{C}$ & Number of consumer agents \\
$N_{P}$ & Number of provider agents \\
$A$ & Size of the geographical area $\left(\mathrm{km}^{2}\right)$ \\
$\alpha_{p l}$ & Path-loss exponent of the geographical area \\
$f$ & Frequency band under consideration $(\mathrm{MHz})$ \\
$S$ & Size of a Basic Bandwidth Unit $(\mathrm{BBU})$ \\
$U$ & Number of BBUs in the unlicensed spectrum band \\
$E$ & Number of BBUs in an exclusive spectrum license \\
$C_{\text {license }}$ & Cost of an exclusive spectrum license \\
\hline
\end{tabular}

where $P_{0}$ is the received power at a reference distance from the transmitter, $d_{r e f} ; P_{t}$ represents the transmitted power, $P_{r}$ the received power; $G_{t}$ and $G_{r}$ are the transmitter and receiver antenna gains; $\lambda$ is the wavelength of the carrier frequency, $f ; d$ is the propagation distance; and $\alpha_{p l}$ is the path-loss exponent.

\subsection{STOPPING CRITERIA}

Sections 6.3-6.7 describe the attributes of the agents, their behavioral methods, and operating environment. Consumer agents are continuously making choices of spectrum access and learning from their experiences, whereas provider agents are exploring alternative options for organizing spectrum access transactions. Thus, the agent-based model evolves over time as a result of interactions among agents who are trying to maximize their surpluses in the dynamic environment. The results of interest are extracted from the model when the system is stable and the agents' decisions are firmly established. Since the research questions require 
an evaluation of consumers' choices, the stopping criteria are based on the consumer agents' decisions. For each run, the agent-based model is terminated when one of the following conditions is satisfied:

1. The probability that consumer $j$ selects the choice $\left\{d_{c_{j}}^{*}, l_{c_{j}}^{*}\right\}$ is greater than or equal to the probability threshold of 0.9 , for all consumers.

$$
\rho_{c_{j}, t}\left(d_{c_{j}}^{*}, l_{c_{j}}^{*}\right) \geq 0.9 \quad \forall j
$$

2. The reference surplus is stable and the selection of choices does not change during the ten latest samples. We use the standard deviation function, $S T D E V()$, to determine the dispersion of data.

$$
\begin{aligned}
& \operatorname{STDEV}\left(s_{j, t}^{r}\right)<=0.001 \\
& \operatorname{STDEV}\left(d_{c_{j}}^{*}\right)=0.0 \\
& \operatorname{STDEV}\left(l_{c_{j}}^{*}\right)=0.0
\end{aligned}
$$




\subsection{RESULTS AND DISCUSSION}

The results and discussion of the agent-based model for secondary use of radio spectrum are presented in this chapter. The model described in Chapter 6 is put into use to understand when and why consumer agents would choose secondary use of spectrum and in what form. First, Section 7.1 describes the parameter setup for the agent-based model. Next, Section 7.2 presents a set of experiments on the existence of secondary use concept which its results are also served as a model validation. Then, Sections 7.3-7.8 discuss the outcomes and statistical testings of secondary use based on different conditions as outlined in research questions Q1-Q6, respectively. Finally, Section 7.9 discusses the implications and limitations of the experimental results.

\subsection{MODEL SETUP}

In all experiments, each spectrum user is represented by a wireless system that consists of a set of wireless transmitters and receivers. We assume that consumer's wireless system is a two-way infrastructure network that comprises one base station and five wireless clients ${ }^{1}$. In contrast, provider's wireless system is modeled as a one-way infrastructure network, since we assume that primary users (i.e., provider agents) share spectrum of their downlink channels ${ }^{2}$. The number of base stations (transmitters) and clients (receivers) of provider agents are varied, depending on experiments. The default values are 0.5625 transmitter per sq. $\mathrm{km}$ and ten receivers per transmitter. These settings represent a medium-level density of

\footnotetext{
${ }^{1}$ Wireless clients refer to wireless devices that communicate with the base station, such as laptops, PDAs, and workstations.

${ }^{2}$ Communications channels from the base station to wireless clients.
} 


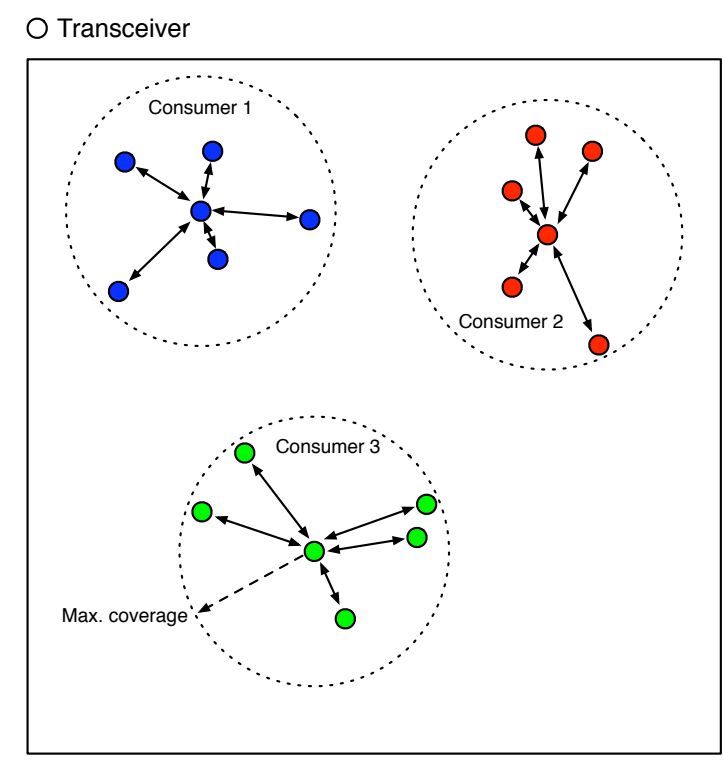

(a)

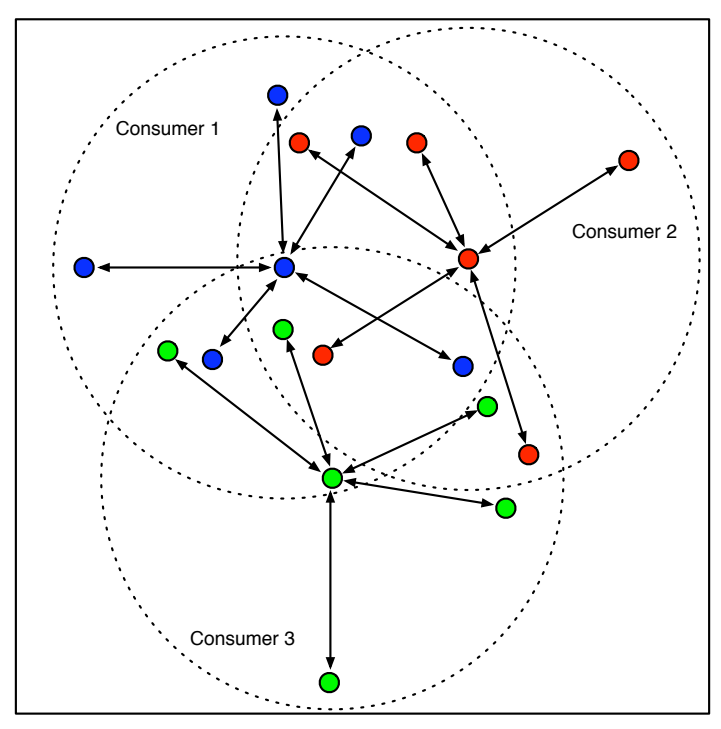

(b)

Figure 7.1: Example of three consumer agents: (a) With small coverage area and (b) With large coverage area.

transmitters and receivers, as specified in the experimental design. In both consumer and provider cases, base stations are randomly placed in the environment and wireless clients are randomly positioned inside the coverage area of their base station. An example configuration of consumer's wireless system is shown in Figure 7.1. The locations of base stations and wireless clients are also randomly changed in every run.

Transmit power is calculated to provide reliable communications (i.e., satisfy receiver's SINR requirement) for any receiver inside the transmitter's coverage area. As mentioned earlier, the utility function of both consumers and providers is defined as:

$$
\mathcal{U}(\gamma)= \begin{cases}\alpha_{u}\left[1-\exp \left(-\left(\gamma-\gamma^{*}\right) / \eta\right)\right] & \text { if } \gamma \geq \gamma^{*} \\ 0 & \text { otherwise }\end{cases}
$$

Unless otherwise specified, we assume that each spectrum user has a hard requirement on the received SINR with $\eta=0.001$, as in Figure 7.2. 


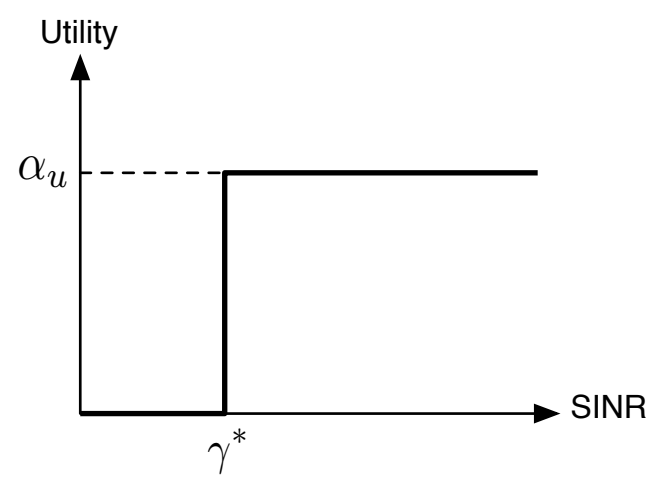

Figure 7.2: Utility function of spectrum users $(\eta=0.001)$.

We also assume that all spectrum users have a potential to behave opportunistically in order to take advantage in a trade. Table 7.1 presents the default values of parameters for all experiments, unless specified explicitly. Results are presented as average values over multiple runs.

\subsection{MODEL VALIDATION AND THE EXISTENCE OF SECONDARY USE}

In this section, we describe the first set of experiments and results of our agent-based model. The objective of these experiments is to produce some baseline results and to provide certain degree of model validation. We study the behavior of consumer agents in terms of their selections of degree of control $\left(d_{c}\right)$ in response to the existence of secondary use, number of consumers in the environment, and spectrum access characteristics of consumer agents.

Section 7.2.1 presents a case where secondary use of spectrum is not allowed, which is the current policy scenario. In this scenario, each consumer agent has to either use unlicensed spectrum or acquire an exclusive license in order to obtain spectrum access. Next, Section 7.2.2 presents the second case where secondary use is introduced. Consumers are allowed to choose intermediate values of degree of control. Here, a larger value for $d_{c}$ means that the spectrum user would prefer advance planning and specific commitments (i.e., 
Table 7.1: Model Parameters

\begin{tabular}{|c|c|c|c|}
\hline Parameters & Values & Parameters & Values \\
\hline $\begin{array}{c}\text { Environment } \\
N_{C} \\
A \\
\alpha_{p l} \\
f \\
\text { Thermal noise }\end{array}$ & $\begin{array}{l}13 \\
16 \text { sq. km } \\
3.5 \\
1,900 \mathrm{MHz} \\
-174 \mathrm{dBm} / \mathrm{Hz}\end{array}$ & $\begin{array}{c}N_{P} \\
S \\
U, E \\
C_{\text {license }}\end{array}$ & $\begin{array}{l}13 \\
200 \mathrm{kHz} \\
50 \mathrm{BBUs} \\
1.0 \text { (normalized) }\end{array}$ \\
\hline $\begin{array}{c}\text { Consumer agents } \\
N_{T} \\
M \\
S E N \\
R E U \\
\alpha_{u} \\
M_{c}^{\text {total }} \\
\mathcal{D}_{c} \\
\mathcal{L}_{c} \\
\beta_{s c} \\
\alpha_{o c} \\
\beta_{p c} \\
T_{b a s e} \\
T_{\text {thres }} \\
G_{t}, G_{r} \\
\text { Receiver noise figure }\end{array}$ & $\begin{array}{l}1 \\
5 \text { BBUs } \\
-100 \mathrm{dBm} \\
\mathrm{N} / \mathrm{A} \\
3.0 \\
10 \mathrm{BBUs} \\
\{0.0,0.25,0.5,0.75,1.0\} \\
\{1,5,10\} \\
0.5 /\left(\alpha_{u}+\alpha_{c}\right) \\
0.1 \\
0.3 \\
0.5 \\
0.3 \\
1 \\
5 \mathrm{~dB}\end{array}$ & $\begin{array}{c}N_{R} \\
D_{\max } \\
\gamma^{*} \\
\eta \\
\alpha_{c} \\
N_{d} \\
N_{l} \\
\alpha_{s c} \\
\delta_{c} \\
\beta_{o c} \\
\gamma_{p c} \\
T_{F} \\
T_{p r o b} \\
d_{r e f}\end{array}$ & $\begin{array}{l}5 \\
250 \mathrm{~m} \\
15 \mathrm{~dB} \\
0.001 \\
1.0 \\
5 \\
3 \\
0.1 \\
0.1 \\
0.2 /\left(\alpha_{u}+\alpha_{c}\right) \\
0.05 \\
0.5 \\
0.5 \\
1 \mathrm{~m}\end{array}$ \\
\hline $\begin{array}{c}\text { Provider agents } \\
N_{T} \\
M \\
S E N \\
R E U \\
\alpha_{u} \\
\text { Spectrum usage } \\
\alpha_{s p} \\
\delta_{p} \\
\beta_{o p} \\
\gamma_{p p} \\
d_{r e f} \\
\end{array}$ & $\begin{array}{l}9 \text { (0.5625 per sq. km) } \\
5 \text { BBUs } \\
-100 \mathrm{dBm} \\
7 \\
3.0 \\
\text { Continuous } \\
0.1 \\
0.1 \\
1 \\
0.05 \\
1 \mathrm{~m}\end{array}$ & $\begin{array}{c}N_{R} \\
D_{\max } \\
\gamma^{*} \\
\eta \\
\alpha_{c} \\
\mathcal{D}_{p} \\
\beta_{s p} \\
\alpha_{o p} \\
\beta_{p p} \\
G_{t}, G_{r} \\
\text { Receiver noise figure }\end{array}$ & $\begin{array}{l}10 \\
500 \mathrm{~m} \\
15 \mathrm{~dB} \\
0.001 \\
1.0 \\
\{0.25,0.5,0.75,1.0\} \\
2 \\
0.1 \\
0.3 \\
1 \\
5 \mathrm{~dB}\end{array}$ \\
\hline
\end{tabular}


Table 7.2: Full Factorial Design for Secondary Use Experiments

\begin{tabular}{c||l}
\hline Factors & Levels \\
\hline \hline Scenario & $\{$ without secondary use, with secondary use $\}$ \\
$D_{\max }$ (meters) & $\{250,500,1000\}$ \\
$\alpha_{u}$ & $\{3.0,6.0,9.0\}$ \\
$N_{C}$ & $\{5,13,19\}$ \\
\hline \# of experiments & $2 \times 3 \times 3 \times 3 \times 30=1620$ \\
\hline
\end{tabular}

greater guarantees) provided by hierarchies or similar structures. When $d_{c}$ is relatively low, it implies that the level of guarantees provided by spot markets or similar structures may be adequate. Table 7.2 presents the design of these experiments.

Finally, Section 7.2.3 demonstrates the effects of incorporating the opportunistic behavior (i.e., another root of transaction costs) into an agent, as described in Section 6.5. In this case, we study the behavior of an opportunistic agent.

\subsubsection{Spectrum Access Without Secondary Use}

In this scenario, we assume that the number of spectrum licenses is greater than the number of consumers. In other words, an exclusive license is available for each and every consumer agent. Figure 7.3 shows the percentage of spectrum access options for different maximum coverage $\left(D_{\max }\right)$ and QoS sensitivity $\left(\alpha_{u}\right)$ of five consumer spectrum users. For comparison, the results of this scenario are displayed together with the case of spectrum access with secondary use, which is discussed in Section 7.2.2.

Since secondary use option is not allowed in this scenario, spectrum access choices are limited to unlicensed spectrum and exclusive licenses and the degree of control value for each consumer is either 0.0 or 1.0. From Figure 7.3, all consumers operate in the unlicensed spectrum when $D_{\max }$ is $250 \mathrm{~m}$ and $\alpha_{u}$ is 3.0. As the QoS sensitivity $\left(\alpha_{u}\right)$ increases, the negative effects of interferences in the unlicensed band start to outweigh the cost of an 


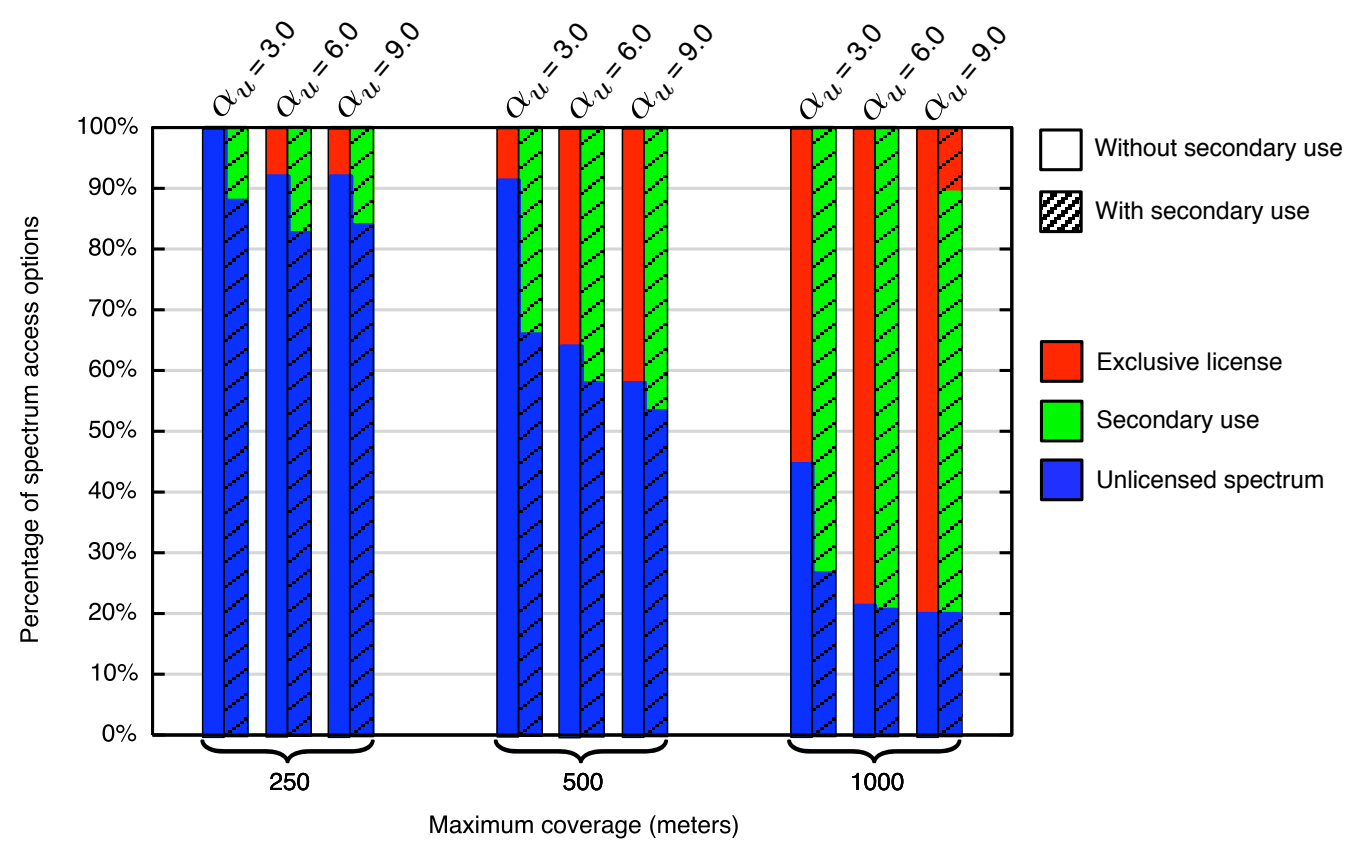

Figure 7.3: Percentage of spectrum access options (for $N_{C}=5$ ).

exclusive license. In other words, these consumers with stringent QoS requirements have less tolerance to interference. As a result, some of the consumer agents switch to exclusive licenses. The percentage of exclusive license is, however, less than $10 \%$.

The graph also shows an increasing use of exclusive license as the maximum coverage of each consumer increases. The larger coverage requires a transmitter to raise its transmit power, thus producing higher interference to other unlicensed spectrum users. At $D_{\max }$ of $500 \mathrm{~m}$, there is an evidence of exclusive license usage as early as $\alpha_{u}$ of 3.0. Once we increases $\alpha_{u}$, the effects of larger coverage result in more than $35 \%$ and $40 \%$ of exclusive license use for $\alpha_{u}$ values of 6.0 and 9.0, respectively. The results continue to follow this trend at $D_{\max }$ of $1000 \mathrm{~m}$. It should be noted that although the unlicensed band can accommodate at least five consumers $\left(U / M_{c}^{\text {total }}=50 / 10=5\right)$, the results show that the exclusive license choice does existed in most of the scenarios. Such an outcome is not unexpected, considering that the unlicensed spectrum does not facilitate any coordination among the unlicensed users. As a result, operating frequencies of unlicensed users may be overlapped and create interferences. 


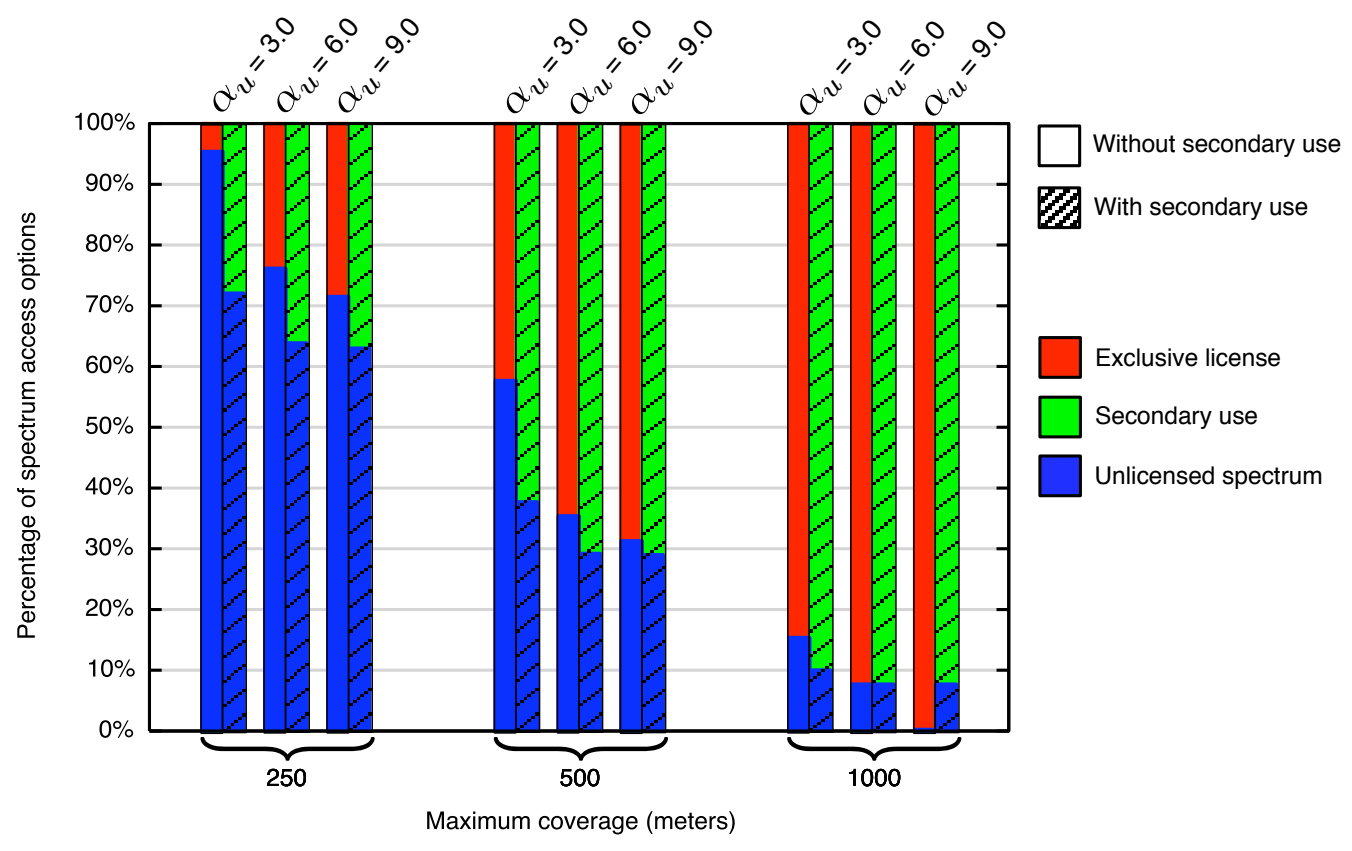

Figure 7.4: Percentage of spectrum access options (for $N_{C}=13$ ).

These interference effects, oftentimes, are too severe that some agents opt for exclusive licenses.

Using the similar design, Figures 7.4 and 7.5 present the results where $N_{C}$ is 13 and 19 , respectively. With an increase in $N_{C}$, the unlicensed spectrum is getting crowded. The figures show that an increase in number of consumers operating in the same area yields a higher use of exclusive licenses as expected. With 13 consumer agents in Figure 7.4, the exclusive license usage appears at all parameter levels, starting from $4.62 \%\left(D_{\max }=250, \alpha_{u}=3.0\right)$ to the highest value of $99.74 \%\left(D_{\max }=1000, \alpha_{u}=9.0\right)$. These percentages are even higher when we have 19 consumer agents in Figure 7.5, ranging from $11.58 \%\left(D_{\max }=250, \alpha_{u}=3.0\right)$ to $100 \%\left(D_{\max }=1000, \alpha_{u}=9.0\right)$. 


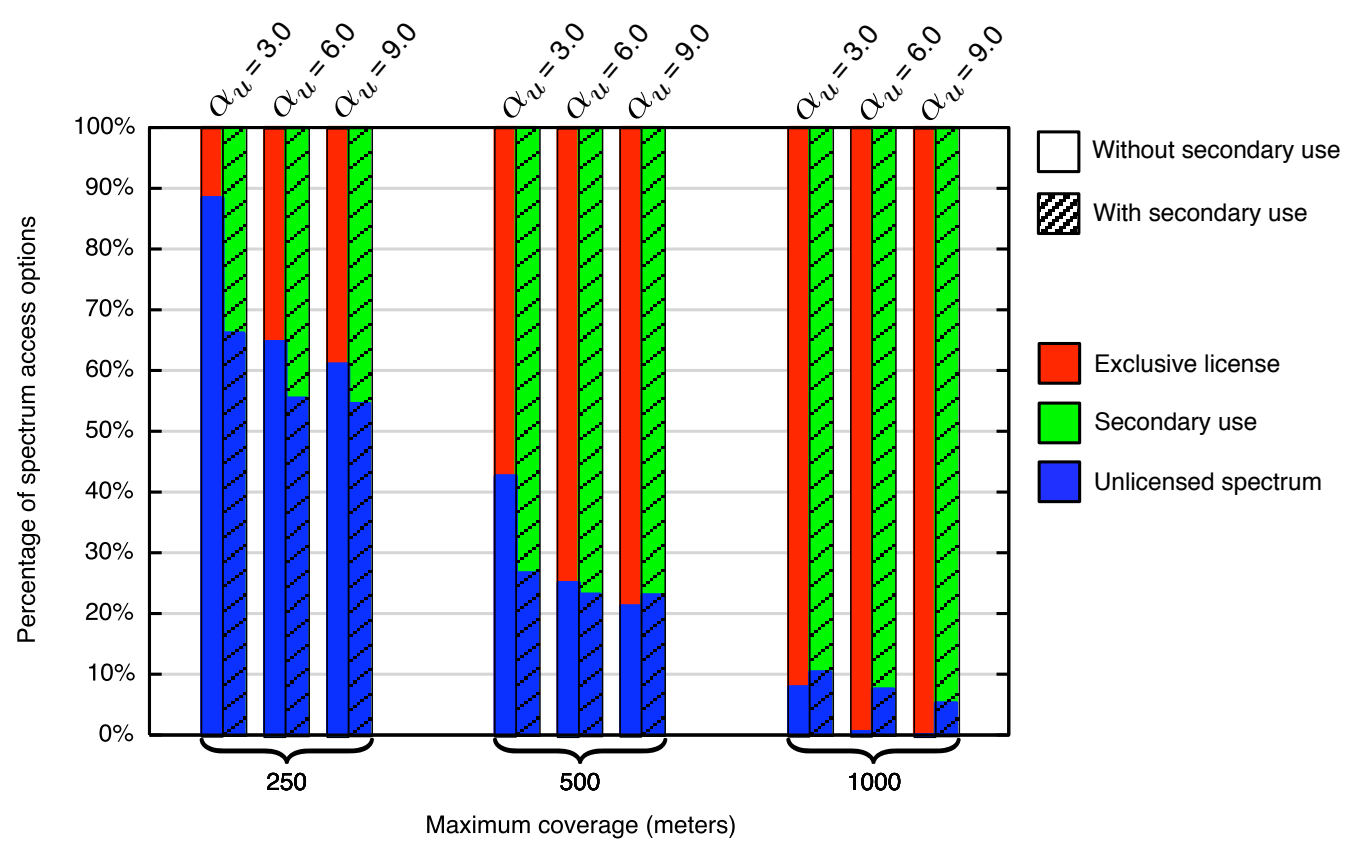

Figure 7.5: Percentage of spectrum access options (for $N_{C}=19$ ).

\subsubsection{Spectrum Access With Secondary Use}

The following set of experiments is similar to the previous one, except that we introduce the concept of secondary use into the model in addition to the unlicensed spectrum and the exclusive licenses. With secondary use, the role of provider agents (spectrum incumbents) comes into play. Each provider holds an exclusive license and shares an unused amount of spectrum to consumers for a period of time, $l_{c} \in \mathcal{L}_{c}$. We assume that the number of provider agents is 19, which represents the maximum level specified in the research question. The effects of number of consumers and providers are studied in research question Q1 in Section 7.3 and thus are not considered here. In this set of experiments, we study the behavior of consumer agents in response to the number of consumers and spectrum access characteristics of consumer agents. The results of this scenario are displayed in a striped pattern along with the results of Section 7.2.2 in Figures 7.3-7.5.

Consider Figure 7.3 at $D_{\max }$ of $250 \mathrm{~m}$ and $\alpha_{u}$ of 3.0 , all consumer agents choose unlicensed 
spectrum when secondary use is not permitted. With secondary use, the result shows that $12 \%$ of consumers choose secondary use and transact with providers. Here, the utility and cost of using secondary use option outweighs the utility of using the unlicensed spectrum. This outcome implies that secondary use is a viable alternative for spectrum access when an exclusive license is considered too expensive. The percentage of secondary use is also higher when $\alpha_{u}$ is increased to 6.0, as expected. The difference between $\alpha_{u}$ of 6.0 and 9.0 is, however, less noticeable because agents who are suffering interference in the unlicensed band have already chosen secondary use.

The amount of secondary use also increases with the maximum coverage. As the coverage increases, consumer agents are more likely to encounter interferences from other agents using the unlicensed spectrum. Therefore, some of them switch to secondary use as an alternative to the crowded unlicensed band and the expensive exclusive license. The results also show that when secondary use option is introduced, it can completely replace the demand for exclusive license, except for one case where $N_{C}=5, D_{\max }=1000$, and $\alpha_{u}=9.0$, which the percentages of exclusive license and secondary use are $10.67 \%$ and $69.33 \%$, respectively. Such results are the effects of opportunism and small numbers [50]. Since the number of consumers is only five and each consumer has a potential to behave opportunistically, these two factors together cannot create enough competitive force to drive secondary use transactions. The trace data confirms that exactly one consumer agent can accommodate the unlicensed band. The other four only select secondary use in some runs or only use exclusive licenses in other runs. In the runs that all of four consumers choose exclusive licenses, the data reveals that providers suffer interference from opportunistic consumers. As a result, they select not to participate in secondary use by choosing a high value of degree of control $\left(d_{p}\right)$. The average $d_{p}$ in these runs are greater than 0.95 . Hence, the four consumers do not have other options but to exercise the exclusive license use. This situation does not occur when the number of consumers is higher, as in Figures 7.4 and 7.5.

Another point of interest is the percentage of unlicensed spectrum use when secondary use is allowed. At $D_{\max }$ of $1000 \mathrm{~m}$, Figure 7.5 shows that the percentages of unlicensed spectrum are higher than those of the case without secondary use. In such scenarios where a large number of consumers have strict QoS requirements and the coverage is also large, 
the unlicensed spectrum becomes over-saturate due to high contention for the shared spectrum resources. Without the secondary use option, this tragedy of the commons render the unlicensed band unusable. As a result, the unlicensed use is dropped to $0.53 \%$ at $\alpha_{u}$ of 6.0 and down to $0 \%$ at $\alpha_{u}$ of 9.0 . On the other hand, when secondary use is introduced, some consumers opt for secondary use, making others experience less contention for the unlicensed spectrum. Thus, the unlicensed band becomes accessible again. In this case, the occupant of the unlicensed spectrum is often the consumer agent who is the first mover into the unlicensed band.

These experiments demonstrate that the agent-based model can produce outcomes corresponding to the real-world spectrum use. Unlicensed spectrum can accommodate a limited number of spectrum users without causing excessive interference. In addition, spectrum users whose application requires a large geographical coverage or demand a high-level QoS tend to acquire exclusive licenses for spectrum access. The introduction of secondary use allows a portion of consumers who cannot afford exclusive licenses to become secondary users. The pre-conditions to secondary use are further examined by research questions (Q1)-(Q6) in Sections 7.3-7.8.

\subsubsection{Secondary Use with Opportunism}

To demonstrate another important feature of the agent-based model, we consider a scenario that contains one provider with an opportunistic behavior. Recall that the provider with opportunistic behavior will try to generate additional revenue by sharing spectrum that could create interferences to consumers (e.g., leasing the same part of spectrum to more than one consumer at a time).

This experiment consists of five consumers with $D_{\max }$ of $200 \mathrm{~m}$. The total number of provider agents, $N_{P}$, is ranging from one to ten providers. We observe the adaptation of the only provider with opportunistic behavior. As discussed in Section 6.5, the opportunistic provider agent uses the reinforcement learning to adapt its probability to act opportunistically. Figure 7.6 presents the probability that this provider will share spectrum that could create interference with his/her transacting partners. The graph shows that the probability 


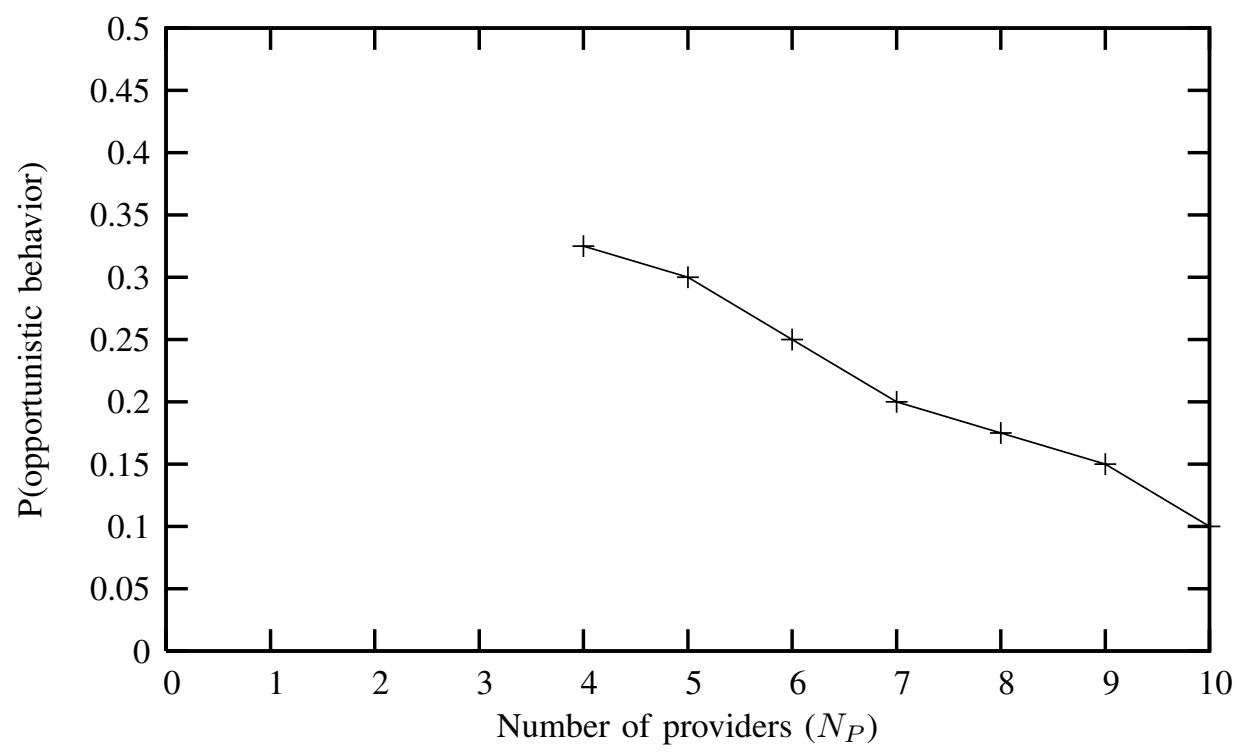

Figure 7.6: Behavior of the opportunistic provider (for $N_{C}=5$ and $D_{\max }=200 \mathrm{~m}$ ).

drops as $N_{P}$ increases ${ }^{3}$.

The result is the market's reaction to opportunism. As the number of providers increases, the amount of sharable spectrum for secondary use becomes larger. Consumer agents have more choices in terms of selecting their transacting partners. According to the trust model in Section 6.5.1, consumers will avoid transacting with providers that create interference. With a large number of providers, they can switch to other providers with an acceptable level of trust. As a result, the provider with opportunistic behavior starts to experience a drop in revenue, because $\mathrm{s} /$ he receives fewer transactions. Consequently, this provider adapts to the situation by reducing his/her opportunistic behavior in order to build trust and acquire more transactions.

\footnotetext{
${ }^{3}$ For $N_{P}=1,2$, and 3 , none of the consumer agents chooses secondary use.
} 
Table 7.3: Parameters for Research Question Q1

\begin{tabular}{c||l}
\hline Parameters & Values \\
\hline \hline$N_{C}$ & $\{1,3,5,7,9,11,13,15,17,19\}$ \\
$N_{P}$ & $\{1,3,5,7,9,11,13,15,17,19\}$ \\
\multicolumn{2}{l}{ Note: See Table 7.1 for other parameters. }
\end{tabular}

\subsection{Q1: NUMBER OF PARTICIPANTS}

In Q1, we examine if the secondary use of spectrum only emerges when there is a large number of participants. Therefore, we perform experiments on the number of consumer and provider agents and measure the percentage of consumers selecting secondary use as the spectrum access option in each experiment. Table 7.3 summarizes factors and values in this experimental design.

Figure 7.7 presents the results of percentage of secondary use for different numbers of consumers and providers in the environment. The figure shows that secondary use increases as the number of consumers and providers increases. With a small number of providers, there is a lack of viable competition. Thus, these providers enjoy monopoly advantages. They can charge the most profitable price that the consumers would accept (i.e., near or at consumer's reservation price). A small number of providers also limit the amount of spectrum available for secondary use. In addition to this, a small number of participants, whether they are consumers or providers, can invoke opportunistic behaviors and result in interference among agents. This is in contrast to the situation where there is a large number of participants. As Williamson [50] observed: “...rivalry among large numbers of bidders will render opportunistic inclinations ineffectual. Parties who attempt to secure gains by strategic posturing will find that such behavior is nonviable." The effects of number of agents on the opportunistic behavior are demonstrated in Section 7.2.3. As a result, consumers may find the secondary use option undesirable when the number of participants in secondary use is low. 


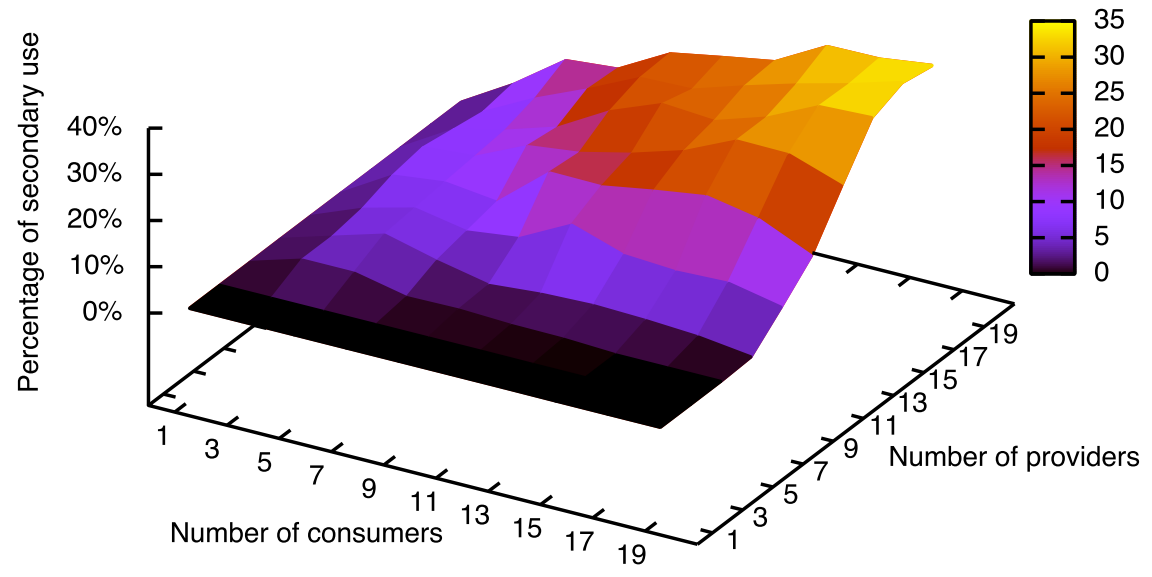

Figure 7.7: Percentage of secondary use vs. Number of consumer and provider agents.

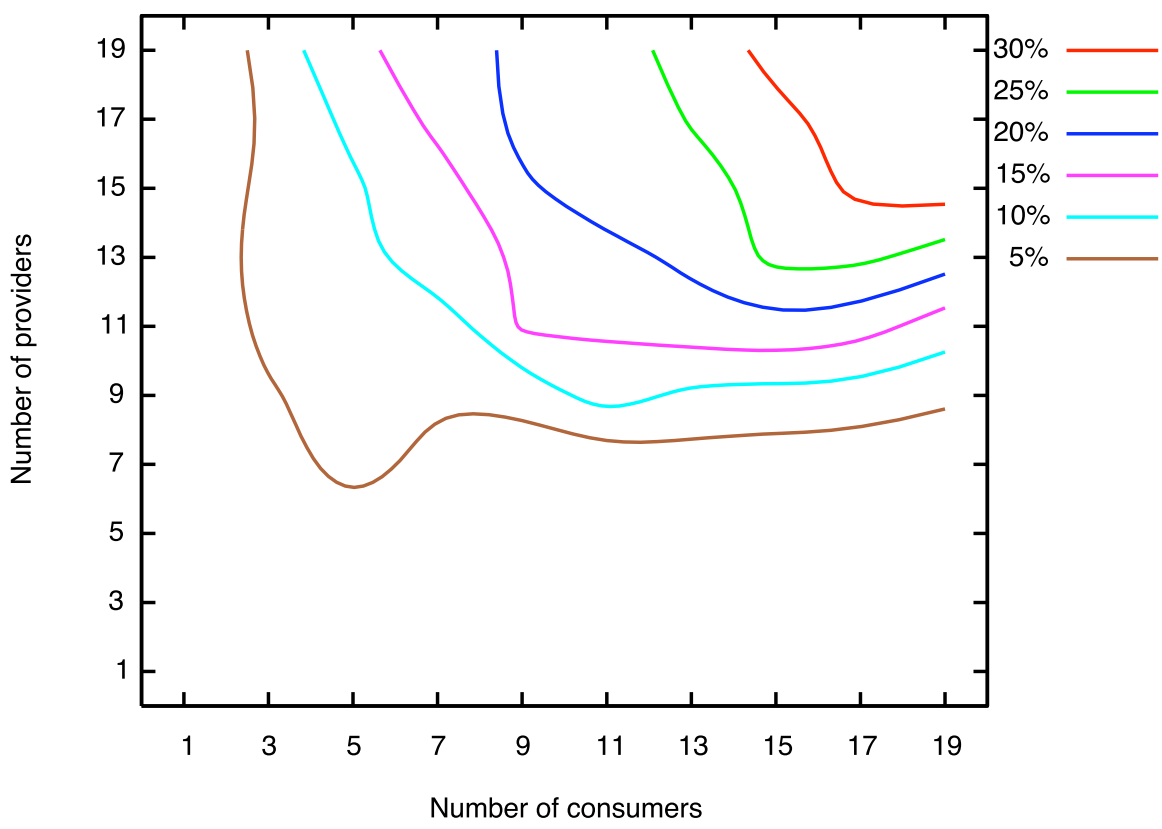

Figure 7.8: Contour lines of the percentage of secondary use. 


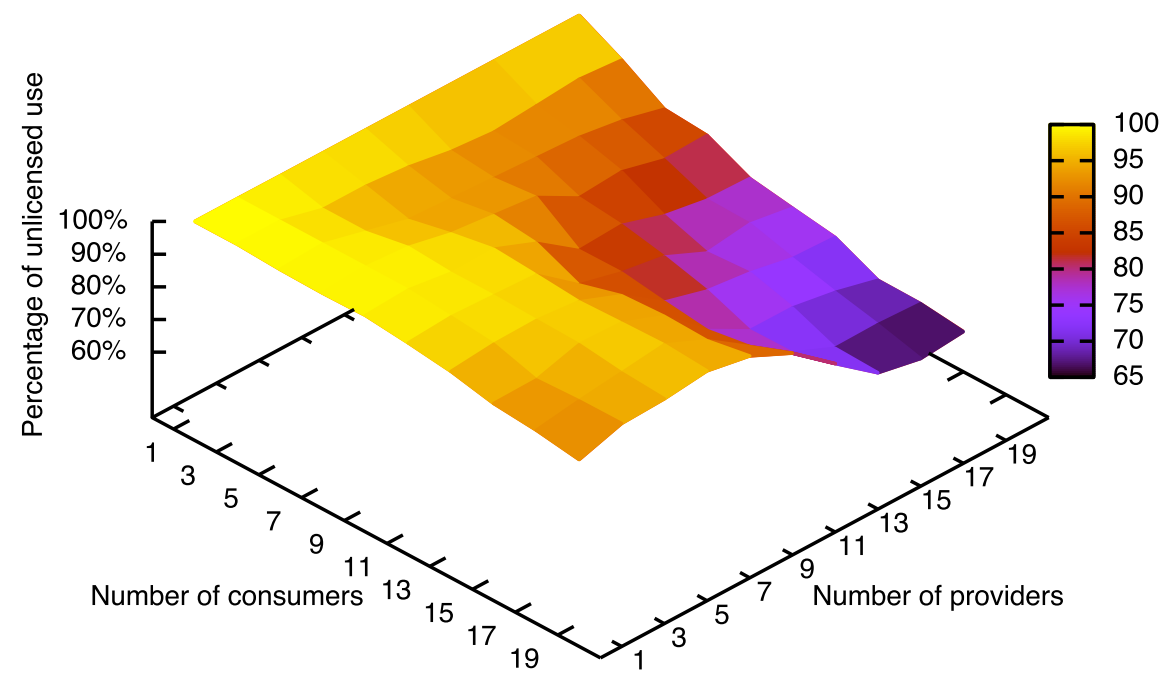

Figure 7.9: Percentage of unlicensed use vs. Number of consumer and provider agents.

Figure 7.8 is a contour plot of percentage of secondary use in Figure 7.7. The contour lines demonstrate that consumer agents are taking advantage of secondary use at a $5 \%$ level even there is a small number of consumers in the environment $\left(N_{C}=3\right)$. However, the $5 \%$ secondary use only emerged when there are at least seven provider agents in the environment. At $N_{C}=9$, the secondary use can reach a $20 \%$ level with $N_{P}$ of at least 15 . The percentage does not go beyond $20 \%$ because the unlicensed spectrum can accommodate the other $80 \%$ without harmful interference. As the number of consumers increases, the percentage of secondary use becomes higher because of the congestion in the unlicensed band. The higher percentage also requires a higher number of providers in the environment. For example, the secondary use can reach a 30\% level when there are at least 15 consumers and 15 providers.

Figures 7.9 and 7.10 display the results of the other two spectrum access options, unlicensed spectrum and exclusive license, respectively. The results of unlicensed spectrum usage show that a small number of consumers (i.e., $N_{C}=1$ and 3 ) select the unlicensed spectrum on average more than $90 \%$ of the time because they can coexist without interference. As 


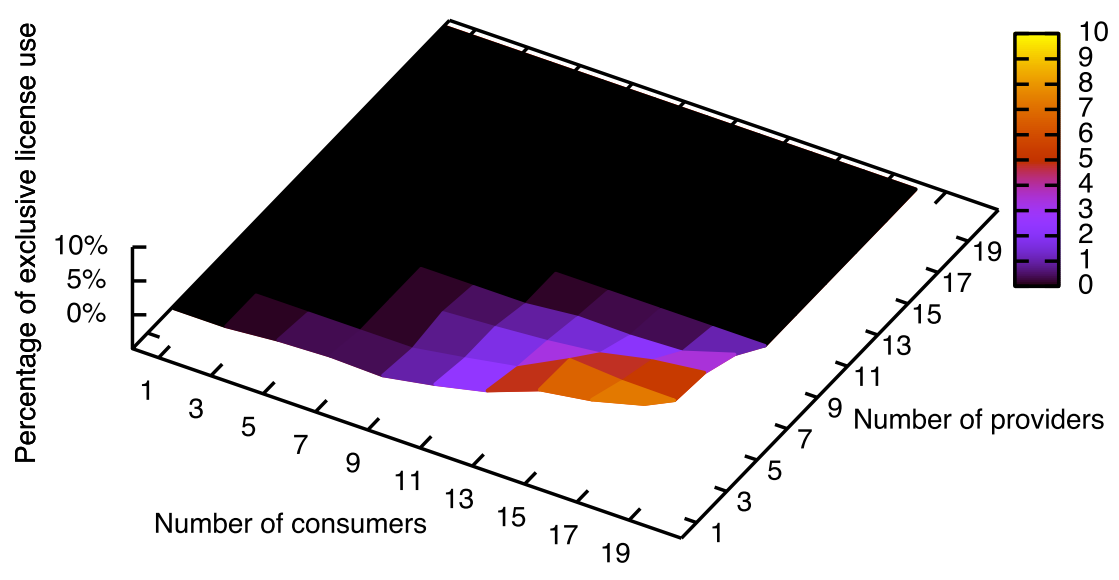

Figure 7.10: Percentage of exclusive license use vs. Number of consumer and provider agents.

$N_{C}$ increases, the unlicensed spectrum gets crowded and the effects of interference starts to outweigh the zero cost. Thus, consumer agents seek other alternatives. From Figure 7.9, the deviation from the unlicensed spectrum (i.e., the percentage drops) occurs in two situations: (1) when the number of consumers and providers increases or (2) when only the number of consumers increases.

The first situation can be explained by comparing Figure 7.9 to Figure 7.7. The increases in $N_{C}$ and $N_{P}$ result in a higher percentage of secondary use. Thus, the unlicensed spectrum usage is reduced. The drop in percentage of unlicensed usage is particularly noticeable when $N_{P} \geq 9$. Similarly, the comparison between Figure 7.9 to Figure 7.10 can describe the second situation. The increase in $N_{C}$ alone when the number of providers is low (i.e., less than nine in most cases) leads to an increasing use of exclusive license. This outcome develops from two factors: (1) unlicensed spectrum is crowded resulting in interferences among users and (2) secondary use is undesirable due to a small number of providers as mentioned earlier. The data shows that consumers start operating in an exclusive license spectrum from $N_{C}=5$. 
Table 7.4: Regression Analysis of Percentage of Secondary Use on Number of Consumers and Providers (Q1)

\begin{tabular}{lcccc}
\hline \multirow{2}{*}{ Variable } & \multicolumn{2}{c}{ Model 1 } & \multicolumn{2}{c}{ Model 2 } \\
\hline$N_{C}$ & $b$ & Beta & $b$ & Beta \\
\multicolumn{1}{c}{$\begin{array}{c}.754^{* * *} \\
(.031)\end{array}$} & .327 & $-.602^{* * *}$ & -.261 \\
$N_{P}$ & & $(.056)$ & \\
& $1.357^{* * *}$ & .588 & .000 & .000 \\
$N_{C} \times N_{P}$ & $(.031)$ & & $(.056)$ & \\
& & & $.136^{* * *}$ & .898 \\
Constant & & $(.005)$ & \\
Adjusted $R^{2}$ & -11.326 & & 2.235 & \\
& & & .567 & \\
\hline
\end{tabular}

Note: $N=3000 ; b=$ unstandardized regression coefficient with standard error in parentheses; Beta $=$ standardized regression coefficient.

${ }^{*} p \leq .05,{ }^{* *} p \leq .01,{ }^{* * *} p \leq .001$

The percentage increases noticeably at $N_{C} \geq 11$ and reaches as high as $9.82 \%$ at $N_{C}=19$.

In order to conduct a statistical testing on Q1, we perform a multiple regression analysis with the percentage of secondary use as a dependent variable and the number of consumers $\left(N_{C}\right)$ and the number of providers $\left(N_{P}\right)$ as independent variables. Table 7.4 presents two regression models for Q1. Model 1 uses $N_{C}$ and $N_{P}$ as independent variables without considering the interaction effect. The result shows that both $N_{C}$ and $N_{P}$ have a significant effect on the percentage of secondary use. The $R^{2}$ value for this model is .452 . Once we incorporate the interaction term, $N_{C} \times N_{P}$, into the regression, Model 2 achieves a higher $R^{2}$ of .567 . From Table 7.4, the regression equation for Model 2 is

$$
\begin{aligned}
\text { Percentage of secondary use } & =2.235-0.602 N_{C}+0.136 N_{C} N_{P} \\
& =2.235+\left(-0.602+0.136 N_{P}\right) N_{C}
\end{aligned}
$$

Both $N_{C}$ and $N_{C} \times N_{P}$ are significant in this model, while $N_{P}$ is not significant. Since the dependent variable measures the choices of consumers, the change in $N_{P}$ alone does 
not effect the percentage of secondary use. However, the increases in both $N_{C}$ and $N_{P}$ result in a higher percentage of secondary use, which confirms our analysis of Figures 7.77.10. The number of consumers alone also significantly influences the secondary use because the capacity and interference effects in the unlicensed spectrum varies with the number of consumers. The regression in (7.2) shows that the increase in $N_{C}$ will produce a higher percentage of secondary use (i.e., the coefficient of $N_{C}$ will become positive) when $N_{P}$ is at least five.

In summary, the statistical testing shows that the secondary use of spectrum only emerges when there is a large number of participants.

\subsection{Q2: OPERATING ENVIRONMENTS}

Research question Q2 concerns with the effects of operating environments on the emergence of secondary use of radio spectrum. We consider the spectrum usage in two scenarios, rural area and urban. Assume that the differences between these two environments are defined by the density of primary user's wireless devices and the path-loss exponent. In a rural area, the primary users have less device density than those in an urban area. Also, the rural area has a lower path-loss exponent compared to the urban area. The experiments in Q2 measure the consumer's choice of spectrum access by varying five levels of provider's transmitter and receiver density and two levels of path-loss exponent. Here, we examine if the proportion of secondary use is higher in a rural environment than an urban environment. The experiments are described in Table 7.5, which is also used by research question Q3 in Section 7.5. Thus, the characteristics of primary user's spectrum usage is also included.

Figures 7.11(a)-(f) illustrate the results of percentage of secondary use for two $\alpha_{p l}$ values in three different scenarios of primary user's spectrum use. The figures show that the percentage of secondary use does not change with an increase in transmitter density $\left(N_{T} / A\right)$. The differences between number of receivers $\left(N_{R}\right)$ is also negligible. In the case of continuous spectrum use, Figure 7.11(a) with $\alpha_{p l}$ of 2.0 shows the secondary use is selected between $30.77 \%$ and $35.38 \%$ of consumer agents. The percentages drop to between $21.54 \%$ 
Table 7.5: Parameters for Research Questions Q2 and Q3

\begin{tabular}{|c|c|}
\hline Parameters & Values \\
\hline$N_{T} / A$ & $\{0.0625,0.25,0.5625,1.0,1.5625\}$ \\
\hline$N_{R}$ & $\{1,5,10,15,20\}$ \\
\hline$\alpha_{p l}$ & 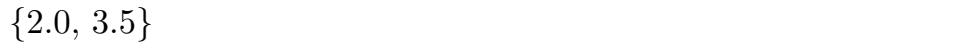 \\
\hline $\begin{array}{l}\text { Spectrum use of } \\
\text { primary users }\end{array}$ & 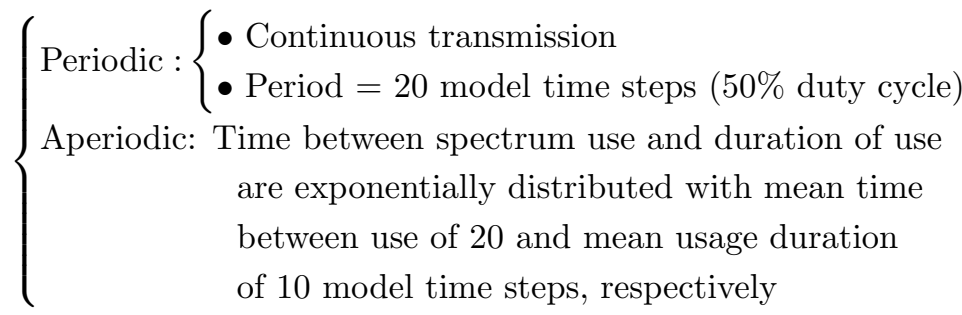 \\
\hline
\end{tabular}

and $26.92 \%$ once $\alpha_{p l}$ is increased to 3.5 in Figure 7.11(b). For periodic spectrum use in Figures 7.11(c) and (d), the percentage is between $12.56 \%$ and $21.54 \%$ for $\alpha_{p l}$ of 2.0 and drops to between $2.05 \%$ and $7.69 \%$ for $\alpha_{p l}$ of 3.5 . The results are similar in the case of aperiodic spectrum use, where the secondary use is selected between $11.54 \%$ and $21.28 \%$ for $\alpha_{p l}$ of 2.0 and between $4.62 \%$ and $7.95 \%$ for $\alpha_{p l}$ of 3.5 .

Although these outcomes suggest that the density of primary user's wireless devices does not affect the consumer's choice of spectrum access, the path-loss exponent $\left(\alpha_{p l}\right)$ appears to lower the percentage of secondary use. Figures 7.12(a)-(c) present a comparison of pathloss exponents on the choices of spectrum access. The figures show that the percentage of secondary use drops with an increase in path-loss exponent. The decrease in secondary use is as much as $11.79 \%, 17.44 \%$, and $16.15 \%$ for continuous, periodic, and aperiodic spectrum use, respectively. The figures also present the percentage of unlicensed use for both pathloss exponents. It appears that the choice of secondary use is, in fact, shifted towards the unlicensed spectrum use as we increase $\alpha_{p l}$ from 2.0 to 3.5. The percentage of unlicensed use is increased in all three scenarios with the maximum changes of $11.79 \%, 17.95 \%$, and $18.2 \%$ for continuous, periodic, and aperiodic spectrum use, respectively. 


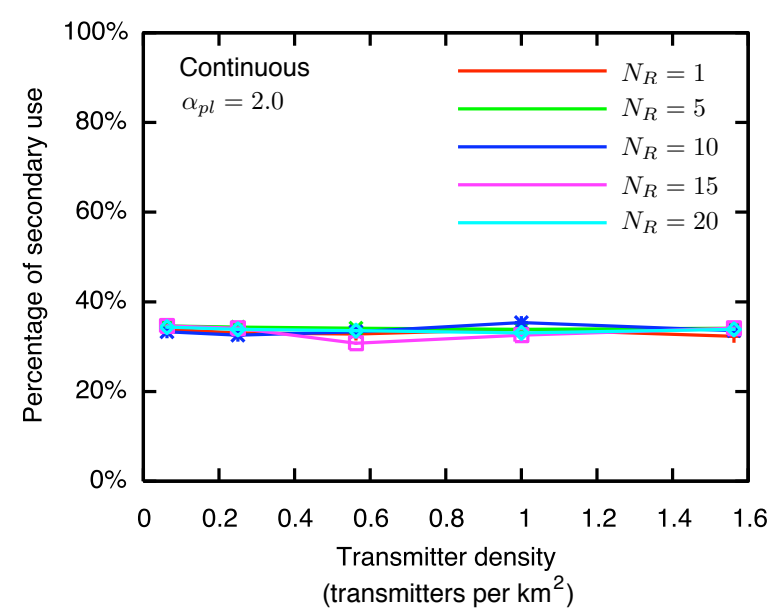

(a)

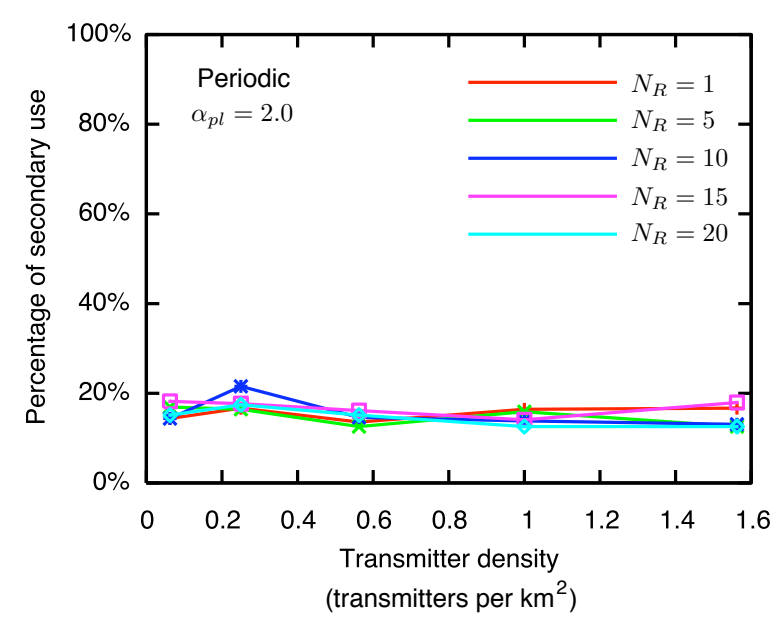

(c)

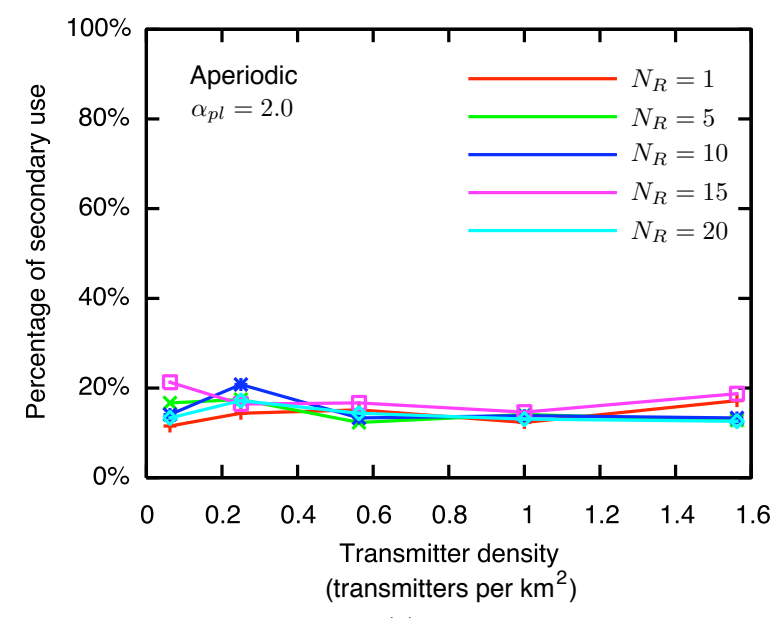

(e)

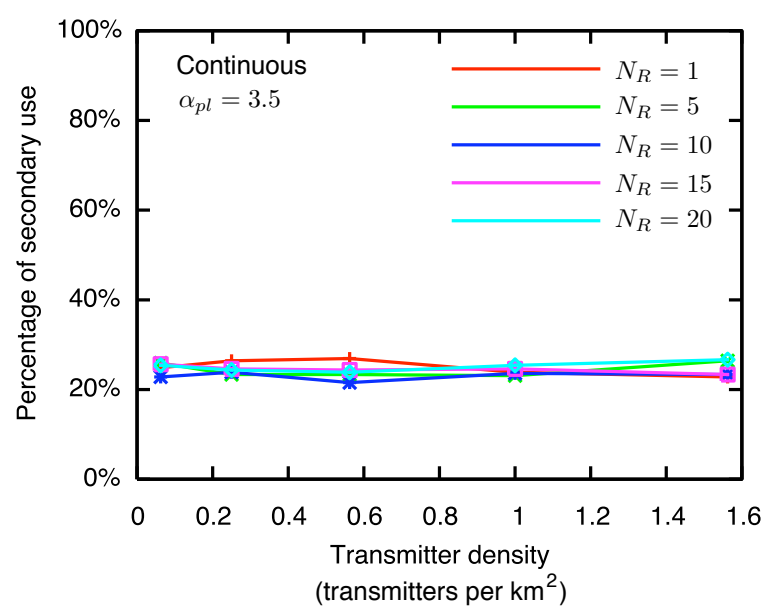

(b)

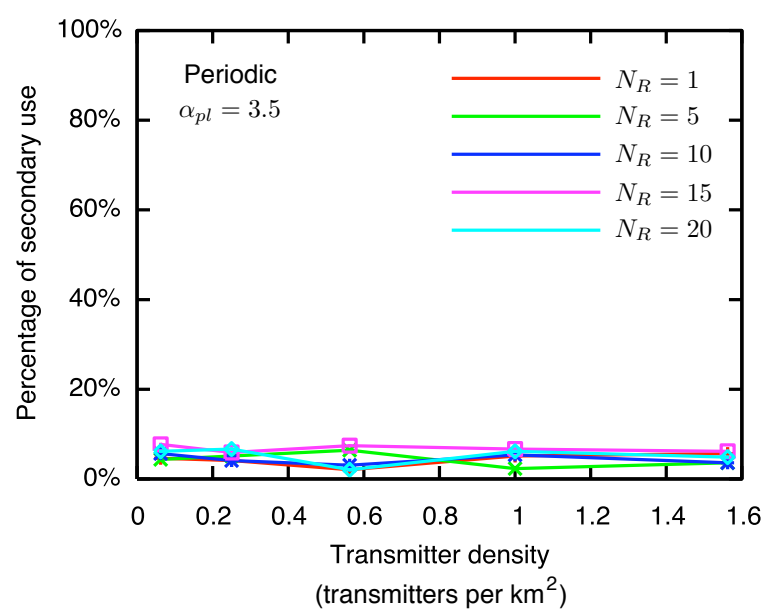

(d)



(f)

Figure 7.11: (a)-(f) Percentage of secondary use vs. Primary user's device density. 


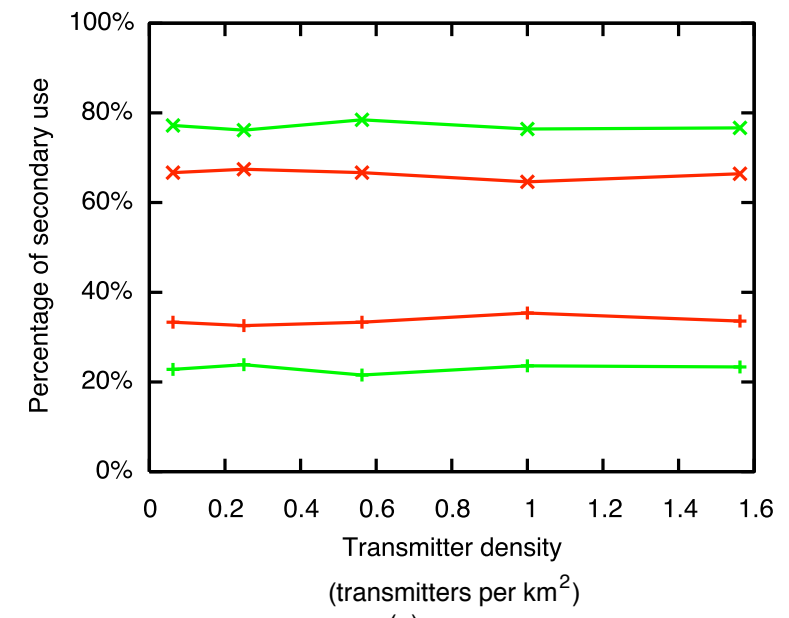

(a)

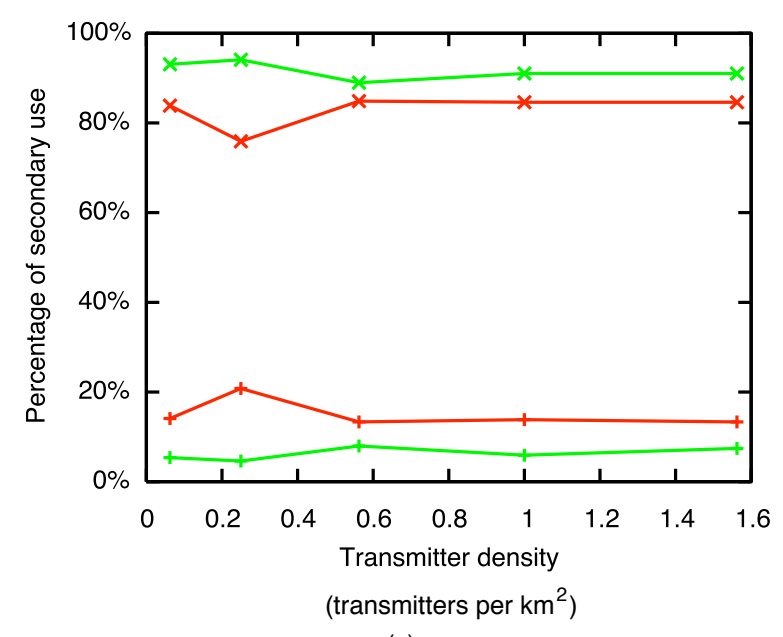

(c)

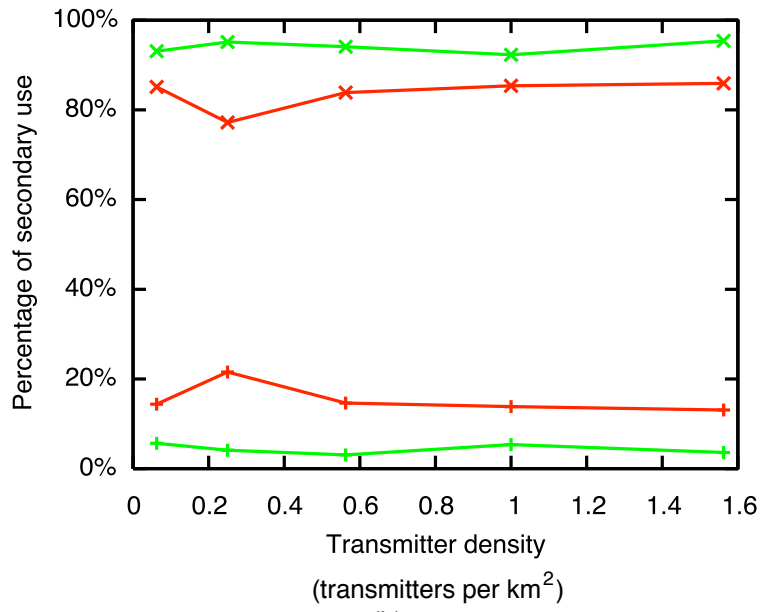

(b)

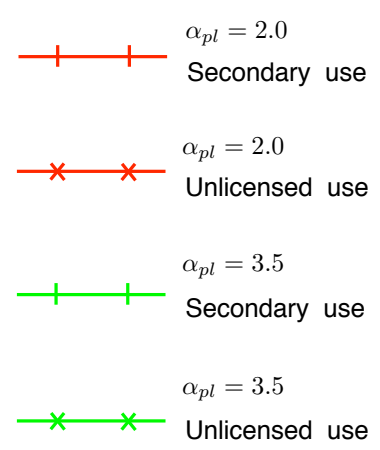

Figure 7.12: Effects of path-loss exponent on the percentage of spectrum access options: (a) continuous, (b) periodic, and (c) aperiodic (for $N_{R}=10$ ). 
Table 7.6: Regression Analysis of Percentage of Secondary Use on Operating Environment Parameters (Q2)

\begin{tabular}{|c|c|c|c|c|c|c|}
\hline \multirow[b]{2}{*}{ Variable } & \multicolumn{2}{|c|}{ Continuous } & \multicolumn{2}{|c|}{ Periodic } & \multicolumn{2}{|c|}{ Aperiodic } \\
\hline & $b$ & Beta & $b$ & Beta & $b$ & Beta \\
\hline 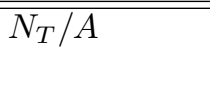 & $\begin{array}{l}-.185 \\
(.457)\end{array}$ & -.009 & $\begin{array}{l}-.96 \\
(.539)\end{array}$ & -.042 & $\begin{array}{l}-.411 \\
(.557)\end{array}$ & -.018 \\
\hline$N_{R}$ & $\begin{array}{l}.006 \\
(.036)\end{array}$ & .004 & $\begin{array}{l}.044 \\
(.043)\end{array}$ & .024 & $\begin{array}{l}.029 \\
(.044)\end{array}$ & .016 \\
\hline$\alpha_{p l}$ & $\begin{array}{c}-6.126^{* * *} \\
(.329)\end{array}$ & -.433 & $\begin{array}{c}-6.974^{* * *} \\
(.388)\end{array}$ & -.421 & $\begin{array}{c}-5.99^{* * *} \\
(.402)\end{array}$ & -.36 \\
\hline Constant & 45.915 & & 29.619 & & 27.062 & \\
\hline Adjusted $R^{2}$ & .186 & & .178 & & .128 & \\
\hline
\end{tabular}

Note: $N=1500 ; b=$ unstandardized regression coefficient with standard error in parentheses; Beta $=$ standardized regression coefficient.

${ }^{*} p \leq .05,{ }^{* *} p \leq .01,{ }^{* * *} p \leq .001$

To explain these results, we conduct a statistical testing on Q2 with the percentage of secondary use as a dependent variable. The independent variables include the primary user's transmitter density $\left(N_{T} / A\right)$, the number of receivers $\left(N_{R}\right)$, and the path-loss exponent $\left(\alpha_{p l}\right)$. Table 7.6 presents the results of three multiple regressions for continuous, periodic, and aperiodic spectrum use scenarios. All three regressions show that only $\alpha_{p l}$ has a significant effect on the percentage of secondary use, whereas $N_{T} / A$ and $N_{R}$ do not influence the choice of spectrum access.

The statistical analysis confirms that the density of primary user's wireless devices does not affect the outcomes. The main reason stems from the implementation of frequency reuse concept in the agent-based model. Frequency reuse allows the same part of spectrum to be used repeatedly across the system. It enables the system to handle a large number of devices with a limited amount of spectrum. Therefore, the amount of spectrum available for secondary use is not severely reduced by a large number of primary user's devices. In addition, this set of experiments uses a large number of provider agents $\left(N_{P}=13\right)$, so consumer 
agents do not need to be concerned about the limited supply of spectrum for secondary use. Conducting experiments with a smaller number of provider agents could generate a different set of results in which the effects of limited supply and potential opportunism could play an important role in selecting the choices of spectrum access. This topic is left for a future work.

The analysis also shows that the effect of path-loss exponent $\left(\alpha_{p l}\right)$ is highly significant. In a rural environment where $\alpha_{p l}$ is low, the signal can propagate farther. Therefore, consumer agents are more likely to experience interference from other signals in the unlicensed spectrum. As a result, some of them switch to the secondary use option. In an urban environment, $\alpha_{p l}$ is relatively high and the signal quality degrades quickly as the distance increases due to obstacles. This environment facilitates the coexistence of devices in the unlicensed spectrum because they are less likely to create harmful interference to each other, given an adequate distance. Hence, the results show a higher percentage of unlicensed spectrum and lower percentage of secondary use associated with a higher value of $\alpha_{p l}$.

In summary, the statistical testing shows that the operating environment does not influence the choices of spectrum access if the definitions of rural and urban area include the density of primary user's wireless devices. However, if the definition is given by the path-loss exponent, the proportion of secondary use is higher in a rural environment than an urban environment.

\subsection{Q3: SPECTRUM USAGE CHARACTERISTICS OF PRIMARY USERS}

In this research question, we study the effects of primary user's spectrum usage characteristics on the consumer's secondary use decision. The provider's characteristics are categorized into two types: (1) constant or scheduled spectrum use and (2) unpredictable spectrum use. The first type represents primary users with predictable spectrum usage such as radio or television broadcasters, whereas the second type attempts to establish inconsistent spectrum usage of public safety users. In Q3, the dependent variable is the consumer's percentage of secondary use and the independent variable is the spectrum usage characteristics of providers. We 
combine the experiments with research question Q2, therefore the experimental design is described in Table 7.5.

The experiments consist of three characteristics of spectrum use by primary users. First, a continuous spectrum use is where the primary user (i.e., provider agent) provides a constant amount of spectrum available for secondary use. Second, a periodic or scheduled spectrum use is modeled as the primary user periodically utilizes his/her own spectrum. In this case, there is a fixed duration (busy duration) in which the primary user needs spectrum access and therefore does not allow secondary use. It is followed by another fixed duration (idle duration) in which the primary user does not utilize spectrum and therefore releases all of his/her spectrum for secondary use. The third characteristic is an aperiodic spectrum use. It is similar to the periodic spectrum use, except that the time between use and duration of use are determined by exponentially distributed random variables. The continuous and periodic characteristics represent a predictable spectrum use or spectrum use defined by a periodic function, as stated in the research question. The aperiodic characteristic represents the unpredictable spectrum use of primary users defined by an aperiodic function.

Figure 7.13 presents the percentage of secondary use for the three spectrum usage characteristics. The effects of transmitter density and number of receivers have already been rejected in research question Q2. Therefore, the focus in this research question is only on the effects of spectrum use by primary users. From the figure, the continuous case achieves a higher level of secondary use with values between $32.56 \%$ and $35.38 \%$. The other two cases have similar results with the percentage of secondary use between $13.08 \%$ and $21.54 \%$ for the periodic case and between $13.33 \%$ and $20.77 \%$ for the aperiodic case.

In order to clarify the results, Figures 7.14 and 7.15 display the results of the other two spectrum access options, unlicensed use and exclusive license use, respectively. The periodic and aperiodic cases show similar results with the unlicensed spectrum use of more than $75.9 \%$, while the continuous case has the unlicensed use from $64.62 \%$ to $67.44 \%$. Figure 7.15 shows that consumer agents in the continuous case do not employ exclusive license as a spectrum access option. The consumers in periodic and aperiodic cases, however, show minimal use of exclusive license (i.e., less than 3.33\%).

The results demonstrate that secondary use is less preferable when the spectrum usage 


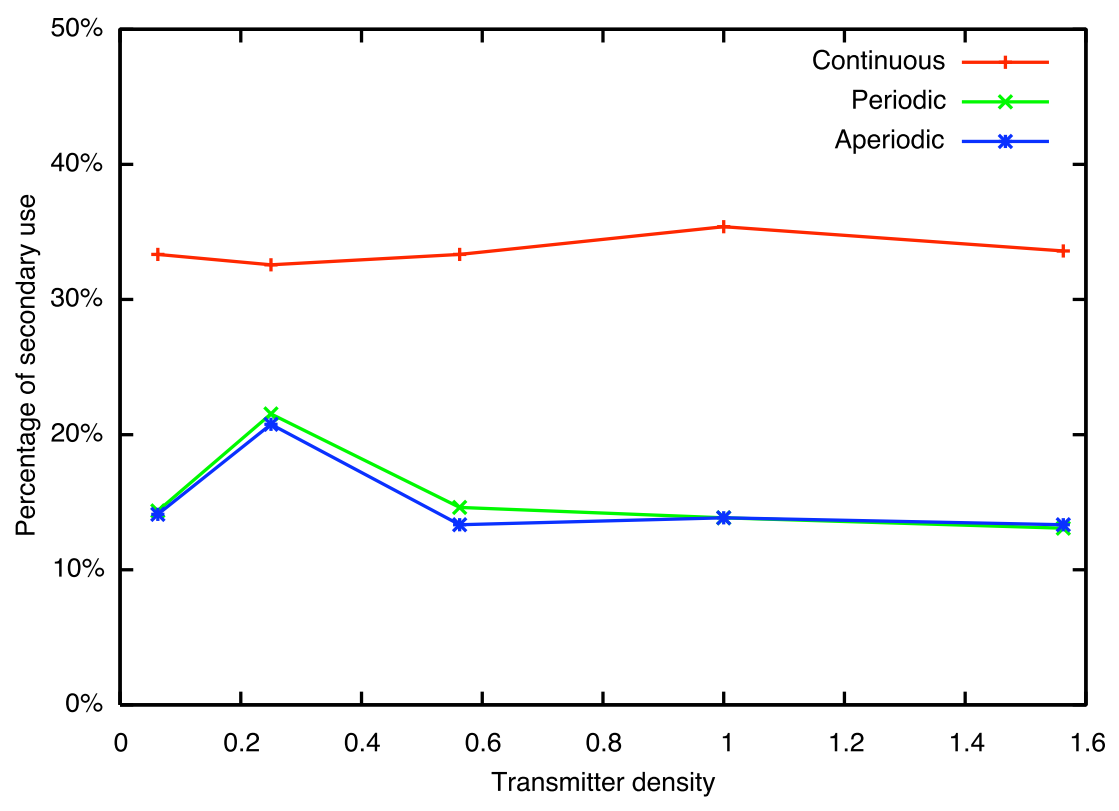

Figure 7.13: Percentage of secondary use vs. Spectrum use of primary user (for $N_{R}=$ $\left.10, \alpha_{p l}=2.0\right)$.

of providers is not continuous. There are two main causes. The first is the fluctuation of the amount of spectrum available for secondary use. With the periodic and aperiodic cases, there is a limited duration in which provider's spectrum is available for secondary use. Once the duration ends, the provider reclaims the spectrum for his/her own use. Thus, the overall spectrum supply for secondary use is fluctuating. Consumers in this case often encounter with a shortage of spectrum supply. The second cause comes from an access duration constraint imposed by provider agents. Providers with periodic or aperiodic spectrum use only accept secondary use transactions with consumers who could vacate the spectrum before the provider's next spectrum access. This constraint implies that the consumer must have a lease duration less than or equal to the provider's remaining idle duration so that s/he can vacate the spectrum before the provider's idle duration expires.

The fluctuation of supply and the constraint on access duration make the secondary use of spectrum less desirable. The results show a higher use of unlicensed spectrum as 


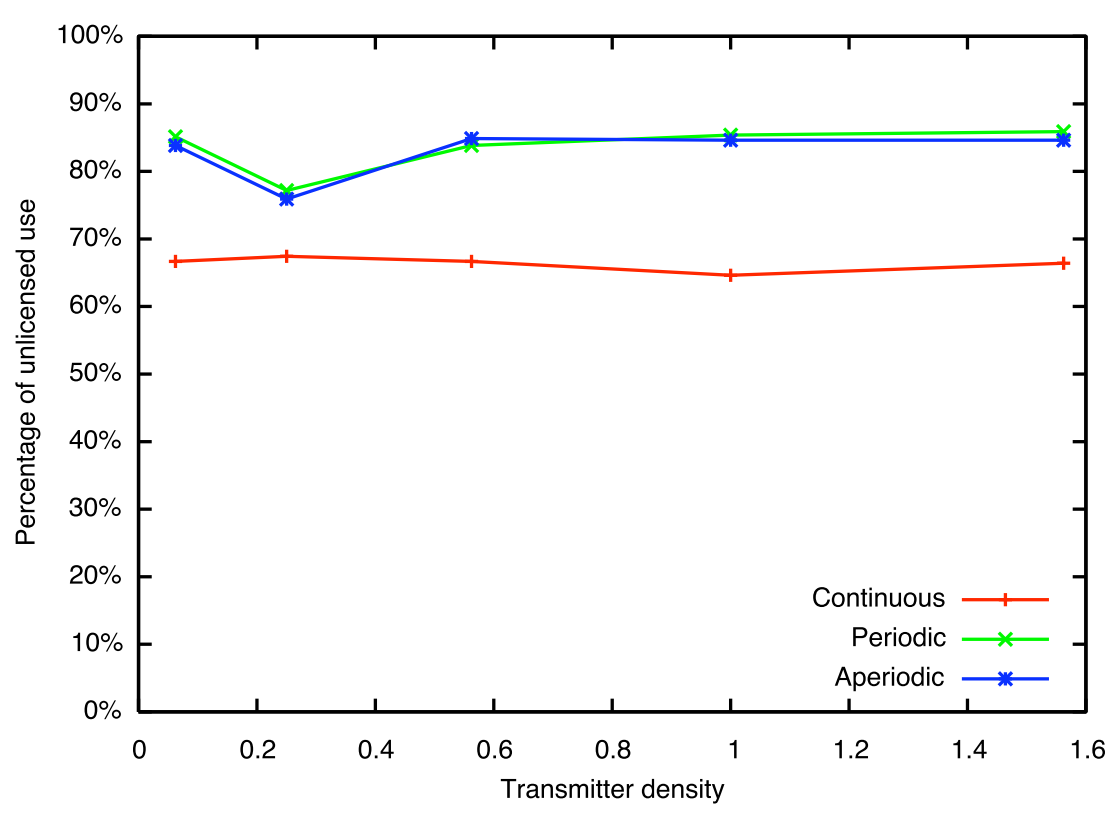

Figure 7.14: Percentage of unlicensed use vs. Spectrum use of primary user (for $N_{R}=$ $\left.10, \alpha_{p l}=2.0\right)$.

a compromised solution for spectrum access. However, as a shared resource, unlicensed spectrum cannot accommodate all consumer agents. Therefore, there is a minor use of exclusive license, as shown in Figure 7.15.

Table 7.7 presents the results of statistical testing with the percentage of secondary use as a dependent variable. The multiple regressions include the primary user's transmitter density $\left(N_{T} / A\right)$, the number of receivers $\left(N_{R}\right)$, the path-loss exponent $\left(\alpha_{p l}\right)$, and types of spectrum use as independent variables. From the research question proposed in Chapter 4, the spectrum usage characteristics of primary users are categorized into two types defined by: (1) a periodic function and (2) an aperiodic function. Hence, the continuous and the periodic spectrum use are classified as a periodic function and the aperiodic use is classified as an aperiodic function. The regression for this case is shown as Model 1 in the table.

The Model 1 regression shows that $\alpha_{p l}$ and the spectrum use as a periodic function have 




Figure 7.15: Percentage of exclusive license use vs. Spectrum use of primary user (for $\left.N_{R}=10, \alpha_{p l}=2.0\right)$.

significant effects to the selection of secondary use ${ }^{4}$. Although the effects of primary user's spectrum use is significant, the analysis of Figures 7.13-7.15 suggests that the significant effect of the spectrum use modeled as a periodic function is biased by the continuous use case. From the figures, the periodic and aperiodic cases exhibit very similar outcomes. Thus, we propose a reorganization of primary user's spectrum use into two types as follows:

Spectrum use of primary users $=\left\{\begin{array}{l}\text { Continuous: } \\ \text { Intermittent: }\left\{\begin{array}{l}\bullet \text { Period }=20 \text { model time steps }(50 \% \text { duty cycle) } \\ \bullet \text { Time between spectrum use and duration of use } \\ \text { are exponentially distributed with mean time } \\ \text { between use of } 20 \text { and mean usage duration } \\ \text { of } 10 \text { model time steps, respectively }\end{array}\right.\end{array}\right.$

The multiple regression of continuous vs. intermittent spectrum use is shown as Model 2 in Table 7.7. The results show that $\alpha_{p l}$ and the continuous spectrum use have significant

\footnotetext{
${ }^{4}$ The influence of $\alpha_{p l}$ is demonstrated and discussed in research question Q2.
} 
Table 7.7: Regression Analysis of Percentage of Secondary Use on Spectrum Usage Characteristics of Primary Users (Q3)

\begin{tabular}{|c|c|c|c|c|}
\hline \multirow[b]{2}{*}{ Variable } & \multicolumn{2}{|c|}{ Model 1} & \multicolumn{2}{|c|}{ Model 2} \\
\hline & $b$ & Beta & $b$ & Beta \\
\hline$N_{T} / A$ & $\begin{array}{l}-.519 \\
(.367)\end{array}$ & -.019 & $\begin{array}{l}-.519 \\
(.300)\end{array}$ & -.019 \\
\hline$N_{R}$ & $\begin{array}{l}.026 \\
(.029)\end{array}$ & .012 & $\begin{array}{l}.026 \\
(.024)\end{array}$ & .012 \\
\hline$\alpha_{p l}$ & $\begin{array}{c}-5.99^{* * *} \\
(.458)\end{array}$ & -.304 & $\begin{array}{l}-.6 .482^{* * *} \\
(.265)\end{array}$ & -.329 \\
\hline $\begin{array}{l}\text { Periodic (1) vs. } \\
\text { Aperiodic (0) spectrum use }\end{array}$ & $\begin{array}{l}10.552^{* * *} \\
\quad(1.6)\end{array}$ & .337 & & \\
\hline $\begin{array}{l}\text { Periodic spectrum use } \\
\times \alpha_{p l}\end{array}$ & $\begin{array}{l}-.561 \\
(.561)\end{array}$ & -.054 & & \\
\hline $\begin{array}{l}\text { Continuous (1) vs. } \\
\text { Intermittent (0) spectrum use }\end{array}$ & & & $\begin{array}{c}17.612^{* * *} \\
(1.308)\end{array}$ & .562 \\
\hline $\begin{array}{l}\text { Continuous spectrum use } \\
\times \alpha_{p l}\end{array}$ & & & $\begin{array}{l}.356 \\
(.459)\end{array}$ & .033 \\
\hline Constant & 27.164 & & 28.328 & \\
\hline Adjusted $R^{2}$ & .187 & & .457 & \\
\hline
\end{tabular}

Note: $N=4500 ; b=$ unstandardized regression coefficient with standard error in parentheses; Bet $a=$ standardized regression coefficient.

${ }^{*} p \leq .05,{ }^{* *} p \leq .01,{ }^{* * *} p \leq .001$ 
effects to the secondary use. Model 2 appears to be a better regression model. The coefficient $b$ of continuous spectrum use is 17.612 , compared to 10.552 for the periodic function in Model 1. Model 2 also produces $R^{2}$ of .457 , while the $R^{2}$ for Model 1 is .187. Both models show no significant effect from the interaction between spectrum usage characteristic and $\alpha_{p l}$

From the statistical results, the secondary use is higher when primary users have consistent and predictable spectrum usage. The consistency and predictability, however, only refers to the case where primary users share a fixed amount of spectrum for secondary use. In the case where spectrum use of primary users is periodic, we believe that consumer agents need to coordinate the timing with provider agents. The synchronization of spectrum access between consumers and providers should eliminate the constraint on access duration, as mentioned earlier. Without the synchronization capabilities, the continuous vs. intermittent spectrum usage appears to be a proper model than the periodic vs. aperiodic usage. We also believe that the effects of the access duration constraint depend on the busy and idle durations of primary users as well as the access duration demanded by secondary users. If the secondary user's access duration is relatively short compared to the duration of primary users, the secondary use should be higher. These topics are assigned to a future work.

\subsection{Q4: COVERAGE REQUIREMENTS}

In this research question, we analyze if consumers who require a large coverage area will obtain spectrum access with a higher degree of control than those with a smaller coverage area. Thus, the experiments in this research question consist of varying five levels of consumer's coverage area $\left(D_{\max }\right)$ as shown in Table 7.8 , which is also used to analyze research question

Q5 in Section 7.7. In this set of experiments, we measure the average degree of control $(\bar{d})$ among consumer agents. As mentioned earlier in Sections 6.2 and 6.3, the degree of control refers to the ability to control opportunistic behaviors and to facilitate coordinations among agents. The unlicensed spectrum yields the lowest degree of control $\left(d_{c}=0.0\right)$, the secondary use provides the intermediate values $\left(0.0<d_{c}<1.0\right)$, and the exclusive license offers the 
Table 7.8: Parameters for Research Questions Q4 and Q5

\begin{tabular}{c||l}
\hline Parameters & Values \\
\hline \hline$D_{\max }($ meters $)$ & $\{10,100,250,500,1000\}$ \\
$\left(\eta, \gamma^{*}\right)$ & $\{(0.001,15),(1.0,10)\}$ \\
Application requirements & $\{$ Rigid, Flexible $\}$ \\
Consumer's $\alpha_{u}$ & $\{3.0,6.0,9.0,12.0\}$ \\
& \\
\hline Note: See Table 7.1 for other parameters.
\end{tabular}

Note: See Table 7.1 for other parameters.

highest degree of control $\left(d_{c}=1.0\right)$. Within the secondary use, the lower the degree of control implies a shift towards a spot market organization, while the higher degree of control suggests a movement towards firms (hierarchy) to organize spectrum access transactions.

Figures 7.16 and 7.17 present the average degree of control by varying the consumer's coverage area $\left(D_{\max }\right)$ for rigid and flexible application requirements, respectively. Both figures show that the average degree of control increases as the coverage area increases. At $D_{\max }$ of $10 \mathrm{~m}$, the coverage is relatively small and the transmit power is relatively low. Therefore, the unlicensed spectrum can be reused more effectively. As a result, the average degree of control values in this case are less than 0.01. Once the coverage is increased, it requires consumer's device to raise its transmit power, which could easily generate harmful interference to other spectrum users. This situation discourages the use of unlicensed spectrum and the secondary use with high potential of opportunistic behaviors. Thus, consumers in this case are shifting towards advance planning and greater guarantees provided by hierarchies or similar structures. Hence, they obtain spectrum access with a higher degree of control. It should be noted that the results of flexible application requirement in Figure 7.17 are similar to the rigid application case. The comparison of rigid and flexible application requirements are given later in Section 7.7.

Figures 7.18(a)-(d) display the percentages of spectrum access choices by varying the coverage area for rigid application requirement. In all cases, the percentage of unlicensed use drops as the coverage increases. The percentage drops are similar in all four cases, implying 


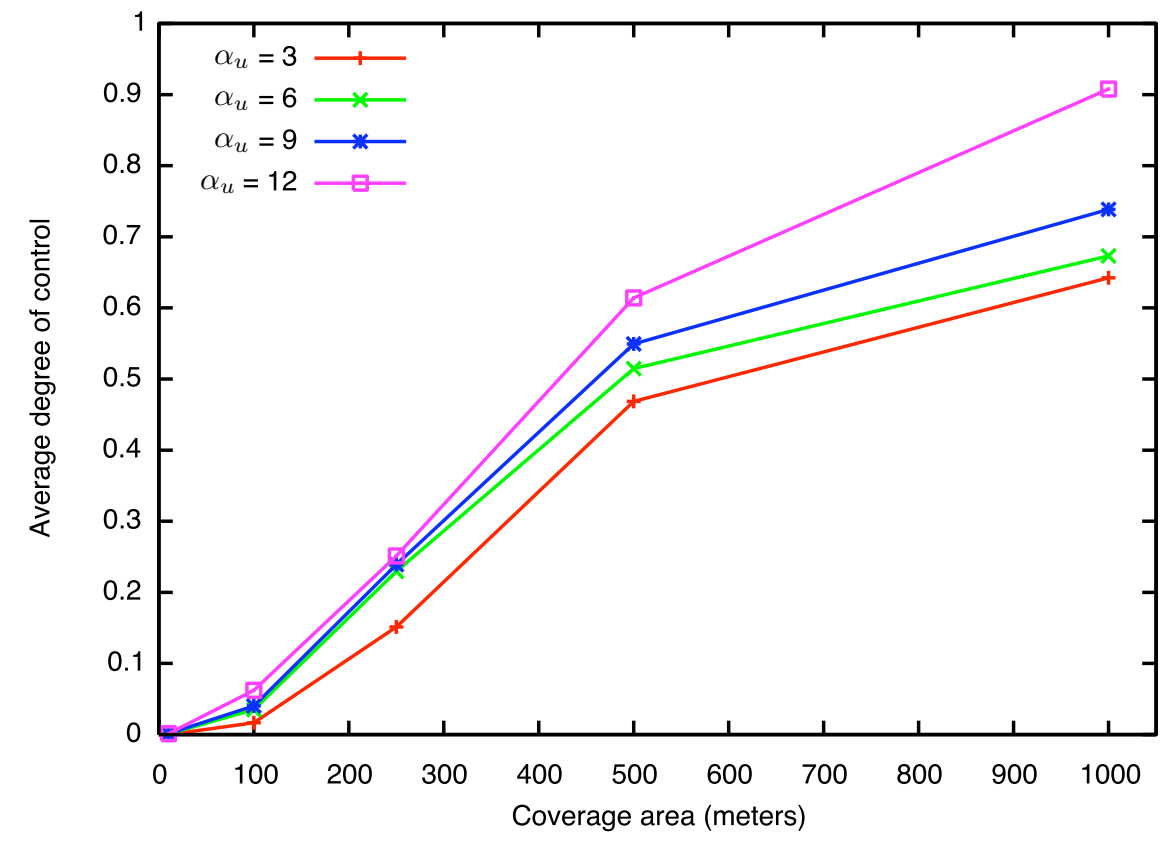

Figure 7.16: Average degree of control vs. Coverage area for rigid application requirement.

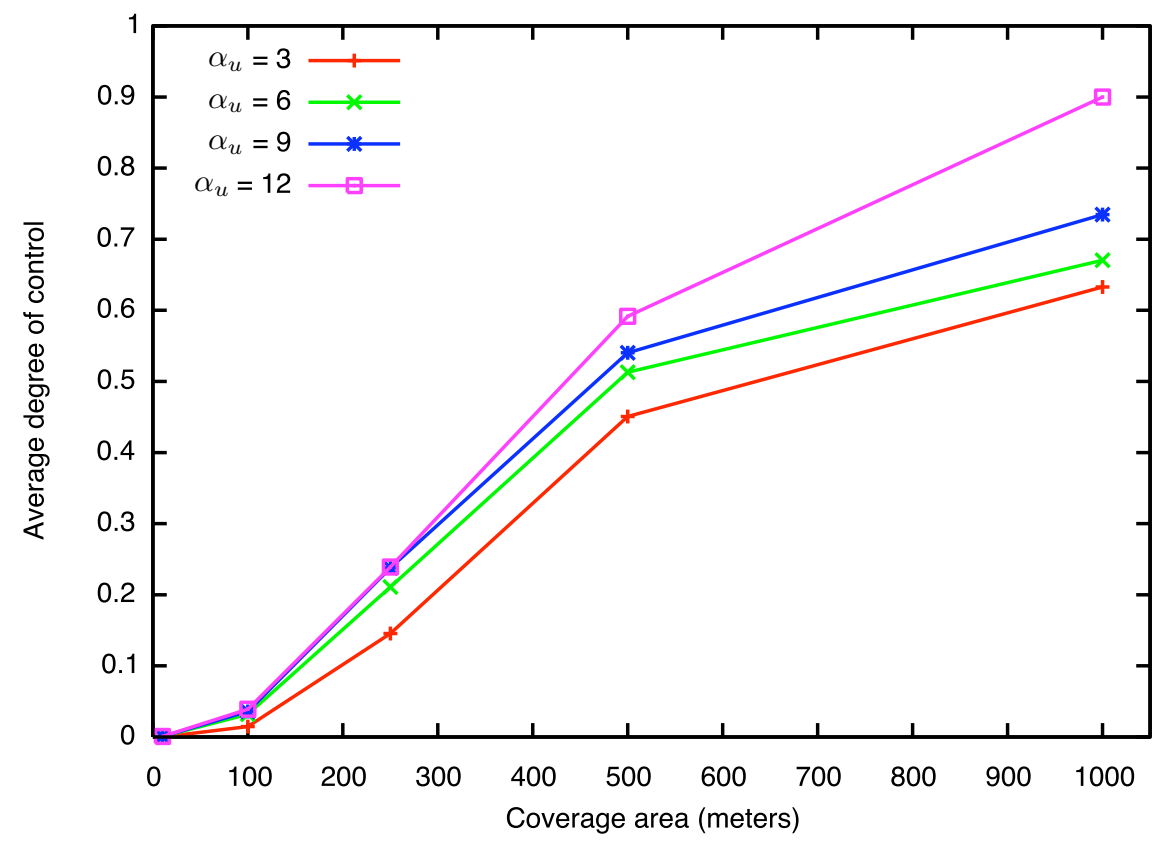

Figure 7.17: Average degree of control vs. Coverage area for flexible application requirement. 


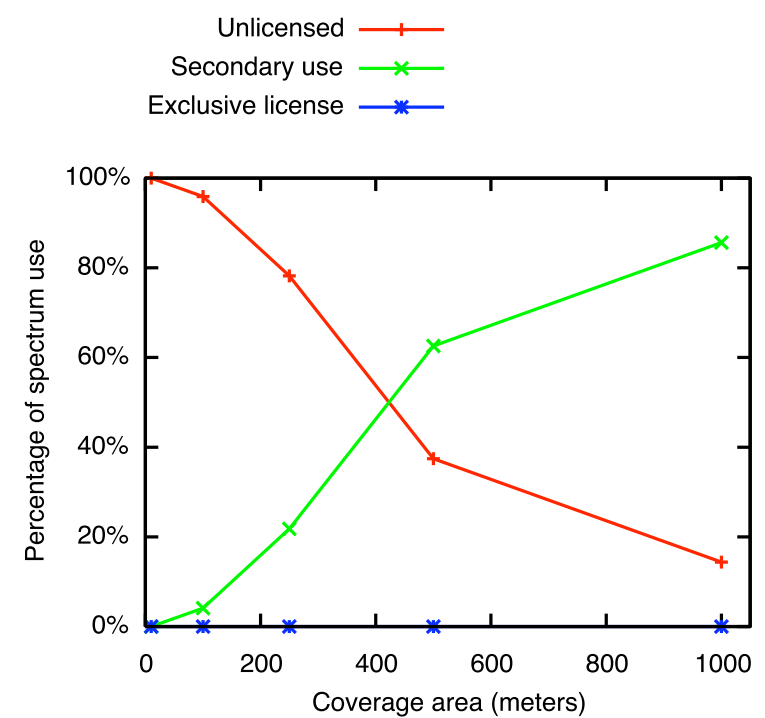

(a)

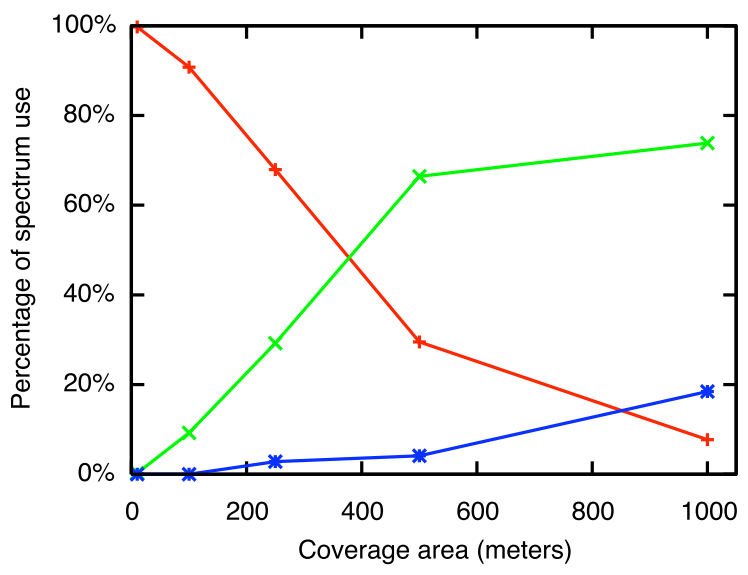

(c)

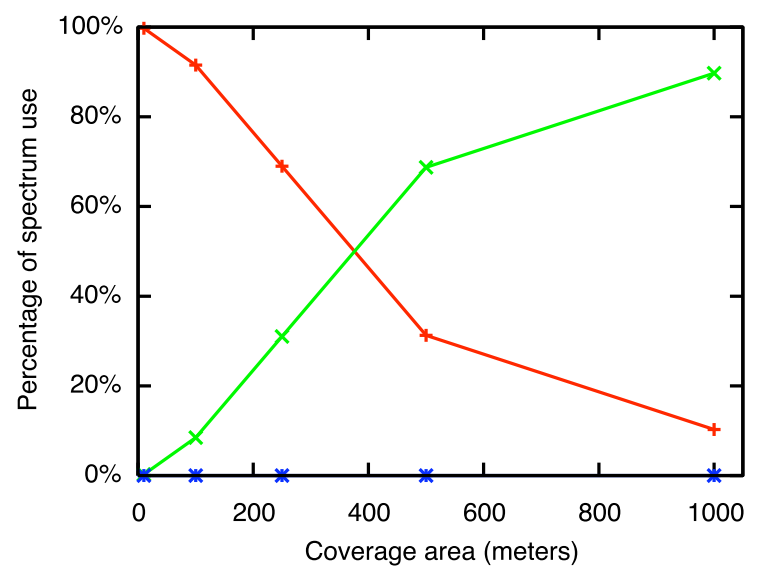

(b)

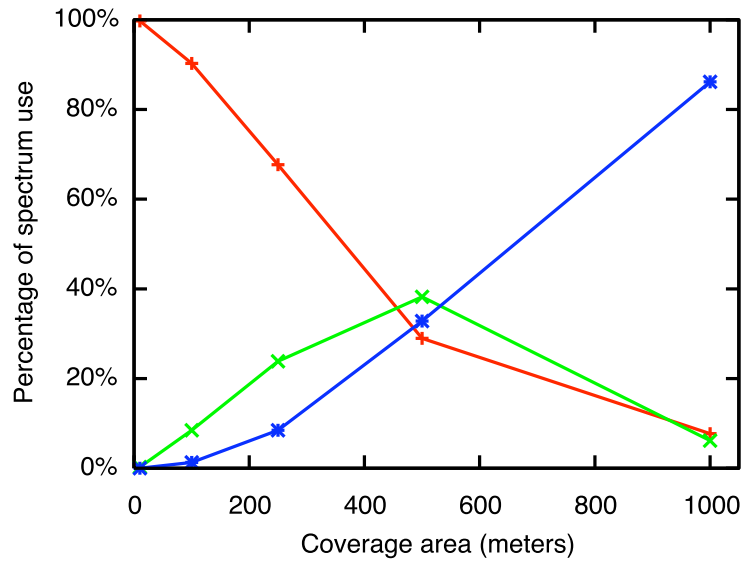

(d)

Figure 7.18: Percentage of spectrum access options vs. Coverage area for rigid application requirement: (a) $\alpha_{u}=3$, (b) $\alpha_{u}=6$, (c) $\alpha_{u}=9$, and (d) $\alpha_{u}=12$. 
that the unlicensed spectrum can accommodate a limited number of users given a coverage requirement. The figures show that the secondary use of spectrum is substituted for the unlicensed use as the coverage increases. Consider Figures 7.18(a) and (b), the percentage of unlicensed use is equivalent to the secondary use at the coverage greater than 400m for consumer's QoS sensitivity $\left(\alpha_{u}\right)$ of 3 and less than 400m for $\alpha_{u}$ of 6 . In Figure 7.18(c) with $\alpha_{u}$ of 9 , the results show $2.82 \%$ of exclusive license use at $D_{\max }$ of $250 \mathrm{~m}$ and continue to increase to $18.46 \%$ at $D_{\max }$ of $1000 \mathrm{~m}$. As $\alpha_{u}$ is increased to 12 in Figure $7.18(\mathrm{~d})$, the exclusive license use begins as early as $D_{\max }$ of $100 \mathrm{~m}$ at $1.28 \%$ and increases to $86.15 \%$ at $D_{\max }$ of $1000 \mathrm{~m}$. The percentage increases of exclusive license are substituted for the secondary use. In particular, Figure 7.18(d) shows that the exclusive license is taking the place of the secondary use to a great extent that the percentage of secondary use drops as $D_{\max }$ is larger than $500 \mathrm{~m}$.

These outcomes develop from two factors. First, the substitution of the secondary use for the unlicensed use is explained by the harmful interference in the unlicensed band. As the coverage increases, the higher transmit power creates interference among unlicensed spectrum users and therefore they switch towards secondary use. Second, as $\alpha_{u}$ increases, consumers are less tolerant to interference. Therefore, the negative effects of the unlicensed band are more intense. In addition, the negative effects of the secondary use (i.e., uncertainty and potential opportunistic behavior) can become significant. With a large coverage area, the provider's opportunistic behavior (i.e., sharing spectrum that is being used by other consumer agents) can be easily detected and produce harmful interferences to the consumers. Especially in the cases of high $\alpha_{u}\left(\alpha_{u}=9\right.$ and 12) and large coverage $\left(D_{\max }=500 \mathrm{~m}\right.$ and $1000 \mathrm{~m}$ ), the cost of an exclusive license justifies the potential negative effects in the unlicensed and secondary spectrum access. Hence, the results show an increase in the percentage of exclusive license use and a decline in the percentages of both unlicensed and secondary use.

The multiple regression analysis with the average degree of control as a dependent variable is presented in Table 7.9. The regression result confirms that $D_{\max }$ has a significant effect on the degree of control, as expected. The interaction effect between $D_{\max }$ and $\alpha_{u}$ is also highly significant. As discussed earlier, the combination of both factors is making the negative effects of unlicensed and secondary use more intense. Therefore, consumers switch to the secondary use with a higher degree of control or an exclusive license. 
Table 7.9: Regression Analysis of Degree of Control on Characteristics of Secondary Users (Q4, Q5)

\begin{tabular}{|c|c|c|}
\hline \multirow[b]{2}{*}{ Variable } & \multicolumn{2}{|c|}{ Model 1} \\
\hline & $b$ & Beta \\
\hline$D_{\max }$ & $\begin{array}{l}.001^{* * *} \\
(.000)\end{array}$ & .681 \\
\hline $\begin{array}{l}\text { Rigid application (1) vs } \\
\text { Flexible application (0) }\end{array}$ & $\begin{array}{l}-.001 \\
(.018)\end{array}$ & -.001 \\
\hline$\alpha_{u}$ & $\begin{array}{l}.001 \\
(.002)\end{array}$ & .008 \\
\hline$D_{\max } \times$ Rigid application & $\begin{array}{l}1.05 \mathrm{E}-005 \\
\quad(.000)\end{array}$ & .011 \\
\hline$D_{\max } \times \alpha_{u}$ & $\begin{array}{c}2.87 \mathrm{E}-005^{* * *} \\
(.000)\end{array}$ & .307 \\
\hline Rigid application $\times \alpha_{u}$ & $\begin{array}{c}.001 \\
(.002)\end{array}$ & .014 \\
\hline $\begin{array}{l}D_{\max } \times \text { Rigid application } \\
\times \alpha_{u}\end{array}$ & $\begin{array}{l}-1.0 \mathrm{E}-006 \\
\quad(.000)\end{array}$ & -.009 \\
\hline Constant & .003 & \\
\hline Adjusted $R^{2}$ & .914 & \\
\hline $\begin{array}{l}\text { Note: } N=1200 ; b=\text { unst } \\
\text { with standard error in par } \\
\text { regression coefficient. } \\
{ }^{*} p \leq .05,{ }^{* *} p \leq .01,{ }^{* * *} p \leq\end{array}$ & $\begin{array}{l}\text { lardized regre } \\
\text { heses; Beta }=\end{array}$ & $\begin{array}{l}\text { coefficient } \\
\text { lardized }\end{array}$ \\
\hline
\end{tabular}


The regression result suggests that spectrum users that require a larger coverage area obtain spectrum access with a higher degree of control than those that require a smaller coverage area. The coverage area also interacts with the user's QoS sensitivity and drives spectrum users towards hierarchies or similar structures to organize spectrum access transactions.

\subsection{Q5: APPLICATION REQUIREMENTS}

The purpose of research question Q5 is to analyze the effects of consumer's application requirement on the average degree of control among consumer agents. In the real-world spectrum usage, different spectrum users can derive different utilities from the same received SINR depending on their spectrum-based applications or services. For example, public safety spectrum users are more sensitive to the received SINR than other spectrum users. Therefore, we perform experiments on the type of application requirements and consumer's QoS sensitivity $\left(\alpha_{u}\right)$. There are two types of application requirements: rigid and flexible applications. Both are described by the parameters $\left(\eta, \gamma^{*}\right)$, which is used to alter the shape of the following exponential utility function:

$$
\mathcal{U}(\gamma)= \begin{cases}\alpha_{u}\left[1-\exp \left(-\left(\gamma-\gamma^{*}\right) / \eta\right)\right] & \text { if } \gamma \geq \gamma^{*} \\ 0 & \text { otherwise }\end{cases}
$$

The experiments in this research question are combined with research question Q4 and is shown in Table 7.8. From the table, rigid and flexible application requirements have the parameters $\left(\eta, \gamma^{*}\right)$ of $(0.001,15)$ and $(1.0,10)$, respectively. Figure 7.19 illustrates the utility functions of both rigid and flexible applications.

Rigid applications, such as a voice service, have a hard requirement on SINR. Such an application requires the received SINR above the threshold $\left(\gamma^{*}=15 \mathrm{~dB}\right)$, otherwise the QoS is unacceptable and users will derive zero utility. Any further increase in SINR above the threshold is indifferent to users of this type of application. On the other hand, flexible applications, such as e-mail or file transfer, can still benefit from a received SINR less than 


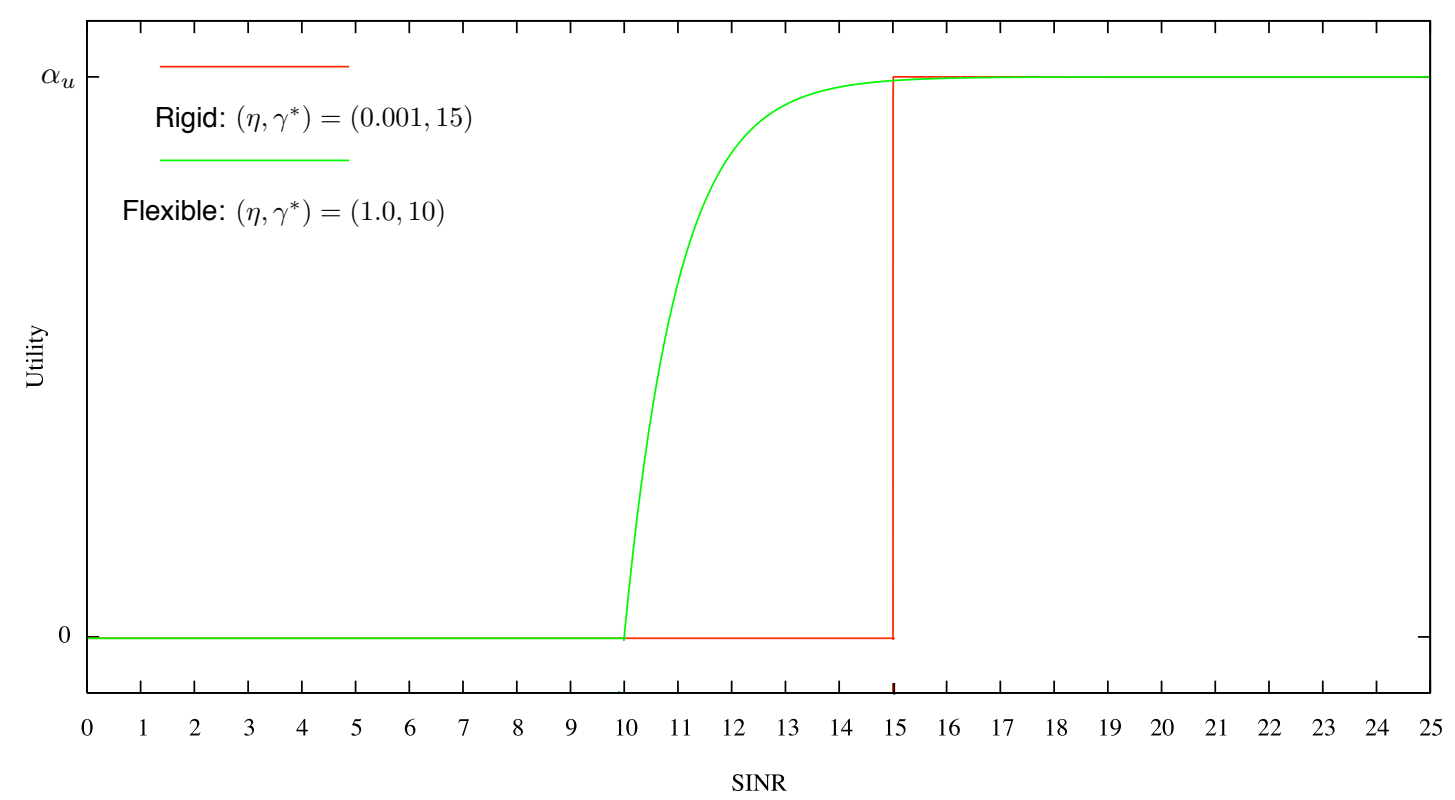

Figure 7.19: Utility functions for H5 experiments: Rigid and flexible application requirements.

$15 \mathrm{~dB}$. From the figure, users of this application acquire positive utility whenever the received SINR is above $10 \mathrm{~dB}\left(\gamma^{*}=10 \mathrm{~dB}\right)$; the utility continues to increase to the maximum utility as the SINR reaches $15 \mathrm{~dB}$, which is the same point as the rigid application type. In both cases, $\alpha_{u}$ defines the maximum utility value and is used to capture the consumer's sensitivity to the received SINR.

We examine if consumers with rigid application requirements and higher sensitivity to the received SINR will obtain a spectrum access option that has a higher degree of control. The experiments in this research question are combined with research question Q4 in Section 7.6. We measure the average degree of control among consumer agents and vary consumer's coverage area, QoS sensitivity, and types of application requirements. The experimental design is provided in Table 7.8.

Figure 7.20 presents a comparison of average degree of control between rigid and flexible application requirements. As discussed in Section 7.6, the coverage area $\left(D_{\max }\right)$ has a significant effect on the degree of control. Therefore, the degree of control is always higher 


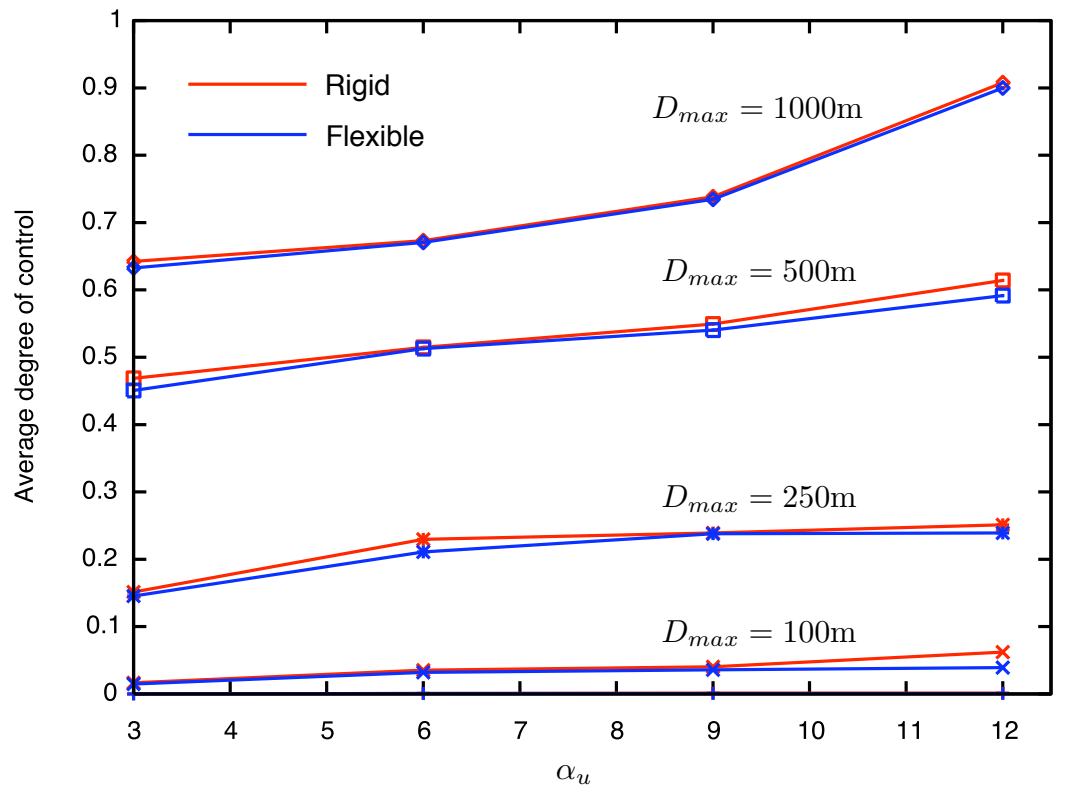

Figure 7.20: Average degree of control: A comparison between rigid and flexible application requirements.

for the larger coverage. The comparison between application requirements shows that there is a small variation between rigid and flexible applications for the same $D_{\max }$ and $\alpha_{u}$. The flexible application obtains spectrum access with a lower degree of control than the rigid application. The soft SINR requirement of the flexible application allows consumers to tolerate a higher level of interference as long as they can derive SINR greater than $10 \mathrm{~dB}$. The regression result in Table 7.9, however, shows that the application requirement does not have a significant effect on the degree of control. We believe that the outcome is a result of the specification of the utility functions. Although the flexible application can acquire a positive utility when the received SINR is greater than $10 \mathrm{~dB}$, the utility cannot reach the maximum value of $\alpha_{u}$ until the received SINR is $15 \mathrm{~dB}$. In addition, the received SINR must be between 10 and $15 \mathrm{~dB}$ in order to create a variation of results between the two. Therefore, adjusting the utility functions of rigid and flexible applications could produce a larger variation. Although the current specification demonstrates a lower degree of control 


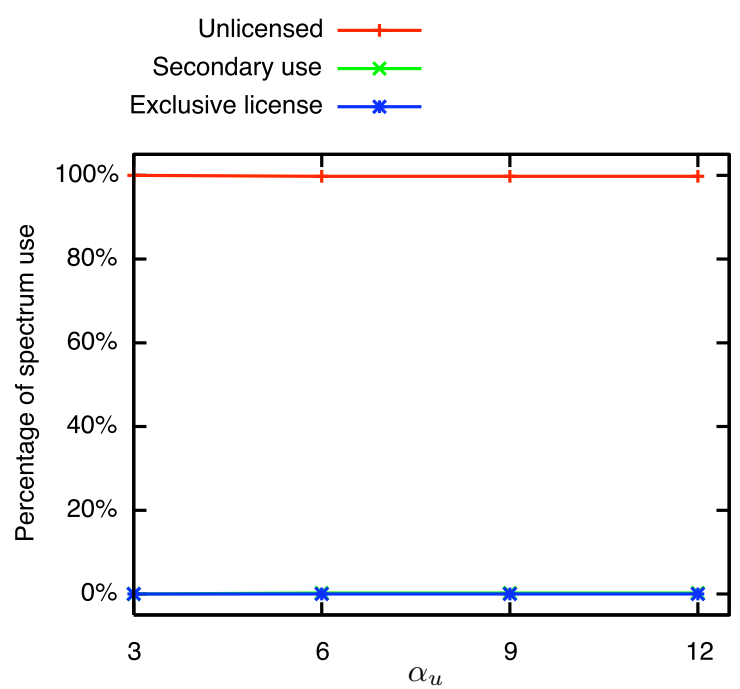

(a)

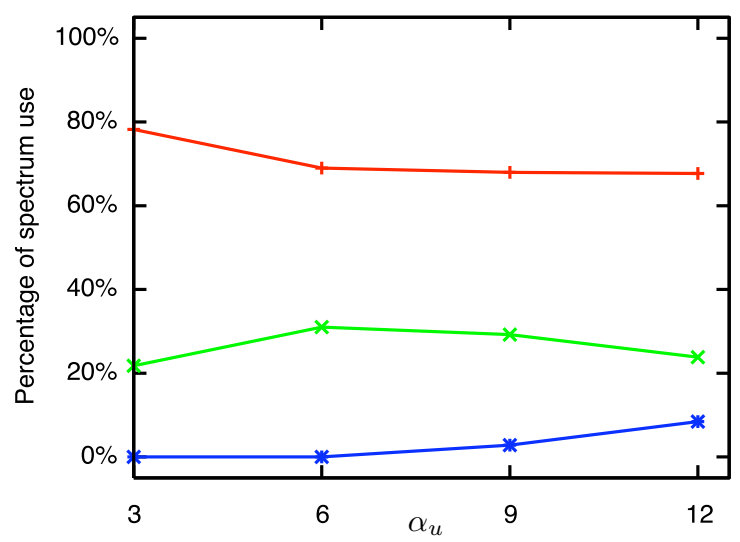

(c)

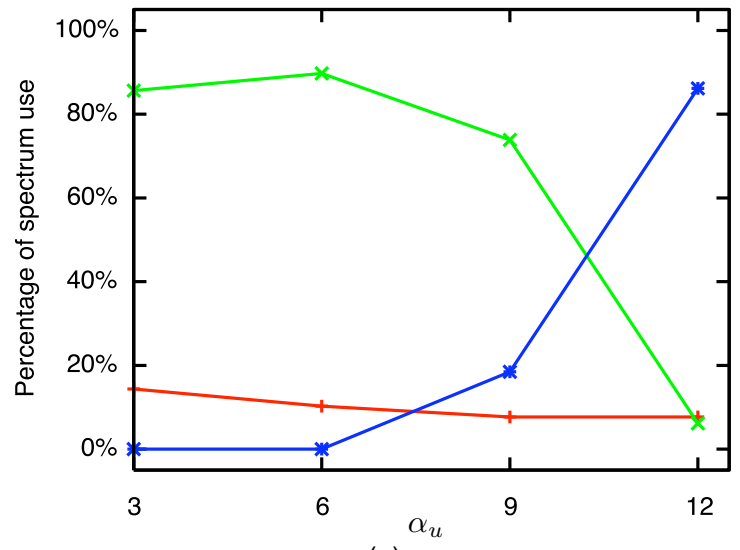

(e)

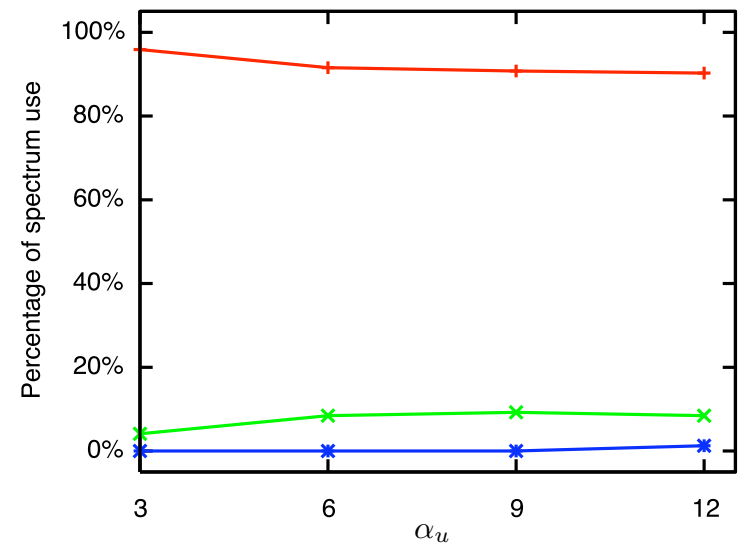

(b)

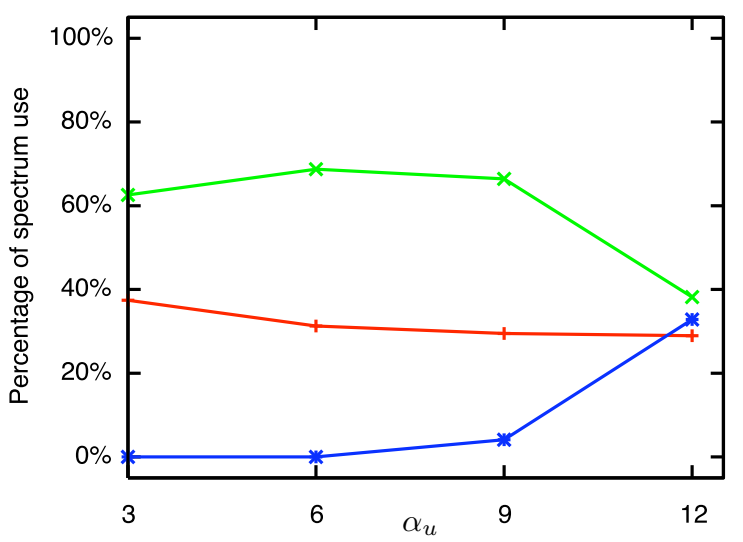

(d)

Figure 7.21: Percentage of spectrum access options vs. $\alpha_{u}$ for rigid application requirement: (a) $D_{\max }=10 \mathrm{~m}$, (b) $D_{\max }=100 \mathrm{~m}$, (c) $D_{\max }=250 \mathrm{~m}$, (d) $D_{\max }=500 \mathrm{~m}$, and (e) $D_{\max }=$ $1000 \mathrm{~m}$. 
for the flexible application, the result cannot be concluded statistically.

Figures 7.21(a)-(e) show the percentages of spectrum access choices by varying $\alpha_{u}$ for rigid application requirement. In Figure 7.21(a), consumers select the unlicensed use more than $99 \%$ and $\alpha_{u}$ does not affect the choices of spectrum access as well as the average degree of control. As the coverage is increased to $100 \mathrm{~m}$ and $250 \mathrm{~m}$ in Figures 7.21 (b) and (c), respectively, the unlicensed use starts to drop and the secondary use is taking the place. In Figures $7.21(\mathrm{~d})$ and (e) with $D_{\max }$ of $500 \mathrm{~m}$ and $1000 \mathrm{~m}$, respectively, the secondary use takes over the unlicensed use and the exclusive license emerges at a high level of $\alpha_{u}$. In all cases, the unlicensed use is relatively constant across the $\alpha_{u}$ values (i.e., the lines are relatively flat in every cases), showing that the unlicensed spectrum can only accommodate a limited number of users. The changes in $\alpha_{u}$ also do not affect the choices of spectrum excess in Figures 7.21(a)-(c). However, Figures 7.21(d) and (e) demonstrate a substitution between the secondary use and the exclusive license, especially at $\alpha_{u}$ of 9 and 12 . The negative effects of the unlicensed and secondary use in a scenario where the combination of $D_{\max }$ and $\alpha_{u}$ is at a high level are already discussed in Section 7.6. The statistical result in Table 7.9 shows that $\alpha_{u}$ by itself does not influence the average degree of control, whereas the interaction between $\alpha_{u}$ and $D_{\max }$ does. The result also shows no significant effect on other interaction terms.

The analysis of result shows that although the flexible application acquires a lower degree of control than the rigid application, they are not statistically different using the current specifications of the utility functions. In the case of user's QoS sensitivity, the sensitivity does not affect the choices of spectrum access as well as the average degree of control. However, once the user's sensitivity is coupled with a scenario where spectrum users require a larger coverage area, spectrum users will move towards organization structures with a higher degree of control to carry out spectrum access transactions. 
Table 7.10: Parameters for Research Question Q6

\begin{tabular}{c||l}
\hline Parameters & Values \\
\hline \hline$U(\mathrm{MHz})$ & $\{10,50,100,150\}$ \\
$D_{\max }$ (meters) & $\{10,100,250,500,1000\}$ \\
$\left(\eta, \gamma^{*}\right)$ & $\{(0.001,15),(1.0,10)\}$ \\
Application requirements & $\{$ Rigid, Flexible $\}$ \\
& \\
\hline Note: See Table 7.1 for other parameters.
\end{tabular}

Note: See Table 7.1 for other parameters.

\subsection{Q6: EXPANSION OF UNLICENSED SPECTRUM}

Throughout this dissertation, we examine the technical, institutional, economic, and strategic issues surrounding the development of secondary use of radio spectrum. Although secondary use is the main subject of this research, it is not the only model for spectrum management. The expansion of the existing unlicensed spectrum also shows a potential to reduce the problem of spectrum scarcity. The concept of expanding the shared commons in conjunction with the market approach for managing spectrum has been advocated by many researchers, such as Lehr [88], Benkler [89], and Lessig [90]. Therefore, we apply the agent-based model to examine the effects of increasing the amount of unlicensed spectrum on the consumer's choice of spectrum access in this final research question.

In Q6, we examine if the additional spectrum in the unlicensed band only benefits spectrum users with flexible application and small coverage requirements. Thus, the experiments consist of varying the amount of spectrum in the unlicensed band $(U)$ and the coverage area $\left(D_{\max }\right)$. Also, we perform experiments on two types of applications: Rigid and flexible, which are described by parameters $\left(\eta, \gamma^{*}\right)$, as in Section 7.7. Table 7.10 summarizes the parameters in this set of experiments.

Figure 7.22 presents the percentage of unlicensed spectrum use vs. the amount of spectrum in the unlicensed band for rigid application requirement. The figure shows an increasing use of unlicensed spectrum as $U$ increases. This outcome corresponds to the results 


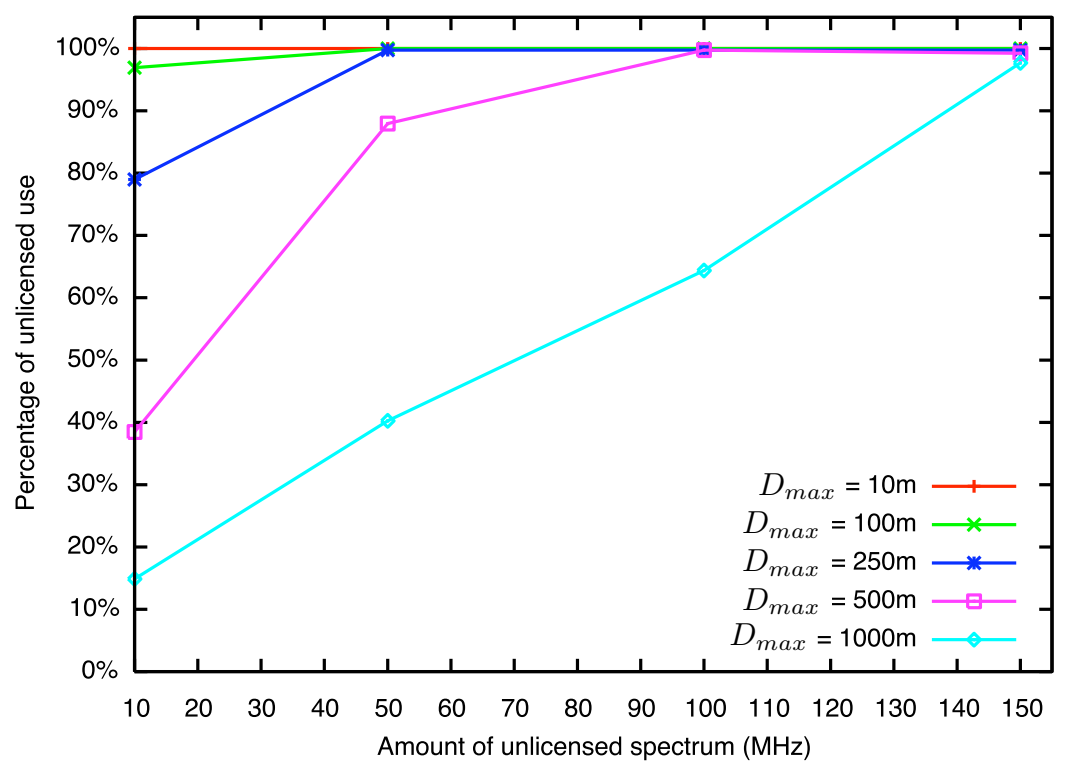

Figure 7.22: Percentage of unlicensed use vs. Amount of unlicensed spectrum for rigid application requirement.

of Sections 7.6 and 7.7, in which the unlicensed band demonstrates a limited capacity to accommodate spectrum users without creating harmful interference. Therefore, an increase in the amount of unlicensed spectrum directly contributes to the capability to support more unlicensed users.

Comparing the results across $D_{\max }$ reveals an interesting observation. At small coverage values $\left(D_{\max }=10 \mathrm{~m}\right.$ and $\left.100 \mathrm{~m}\right)$, an increase in $U$ does not significantly affect the percentage of unlicensed users, since most of the consumers are already using the unlicensed spectrum. At $D_{\max }$ of $250 \mathrm{~m}, 50 \mathrm{MHz}$ of the unlicensed spectrum can support more than $99 \%$ of consumer agents. At $D_{\max }$ of $500 \mathrm{~m}$, the percentage increases significantly from $38.46 \%$ to $87.94 \%$ as $U$ increases from $10 \mathrm{MHz}$ to $50 \mathrm{MHz}$. Similarly, the percentage moves up from $14.87 \%$ to $40.26 \%$ at $D_{\max }$ of $1000 \mathrm{~m}$. From the figure, the overall trend indicates that the percentage of unlicensed use starts as a concave function when $D_{\max }$ is low and transforms into a linear function as $D_{\max }$ increases. The results imply that consumers with lower coverage requirements can derive greater benefits from the expansion of unlicensed spectrum 


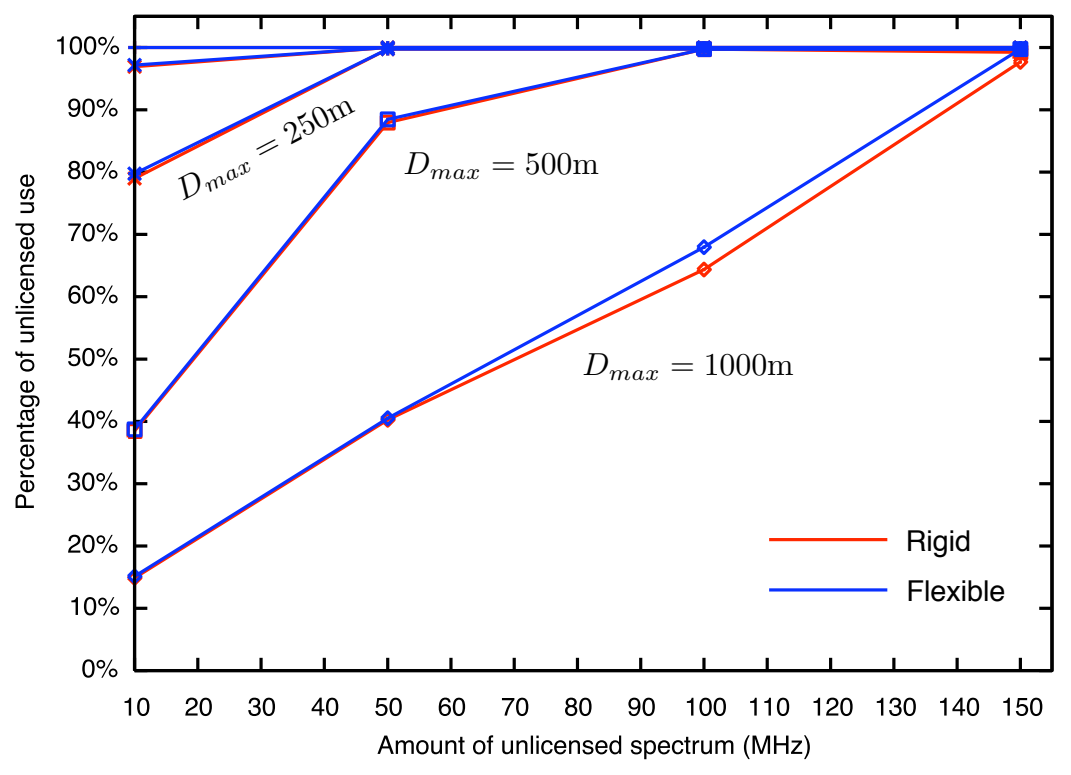

Figure 7.23: Percentage of unlicensed use: A comparison between rigid and flexible application requirements.

than those with higher coverage requirements.

A comparison between rigid and flexible application requirements are presented in Figure 7.23. Both rigid and flexible applications exhibit almost identical percentages of unlicensed spectrum use. The only exception is the case of $D_{\max }=1000 \mathrm{~m}$, in which the flexible application obtains 3\% higher for the unlicensed spectrum access, as expected. The soft requirement on the received SINR of the flexible application allows consumers to tolerate a higher level of interference in the unlicensed spectrum band. The differences between the utility functions of both applications, however, are not large enough to create a significant variation between the two outcomes. We believe that minor adjustments of the utility functions could produce a significant effect on the result of Figure 7.23.

Figures 7.24(a)-(e) shows the percentages of spectrum access choices by varying the amount of unlicensed spectrum for rigid application requirement. In Figure 7.24(a), an increase in $U$ does not affect the choices of spectrum access. As $D_{\max }$ is increased to $100 \mathrm{~m}$ and $250 \mathrm{~m}$ in Figures 7.24(b) and (c), respectively, the unlicensed users experience a higher 


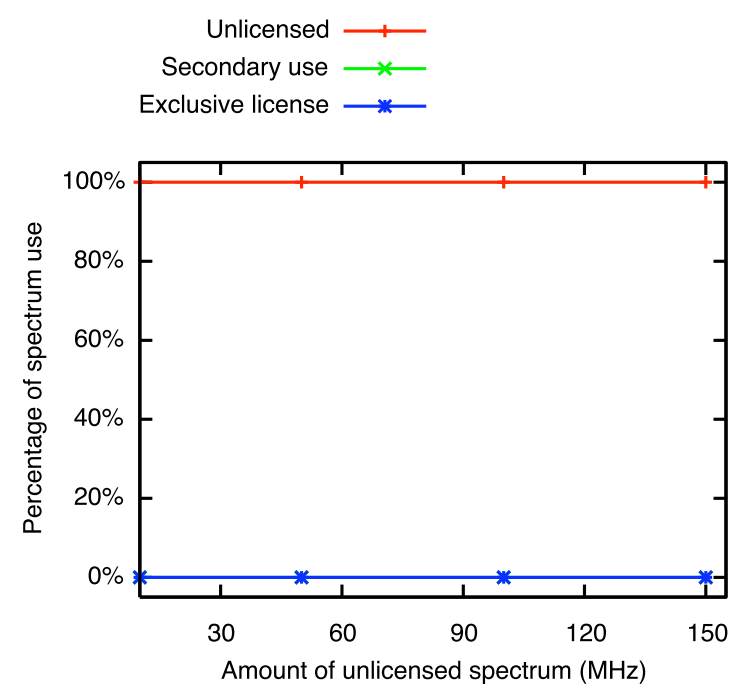

(a)

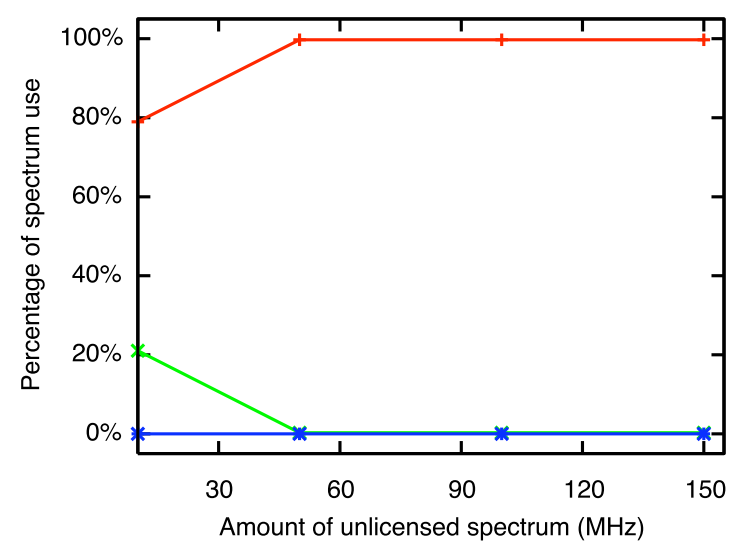

(c)

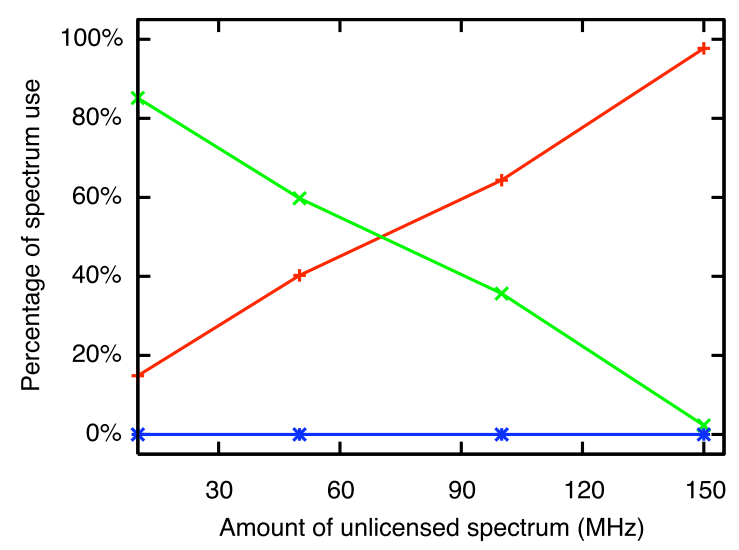

(e)

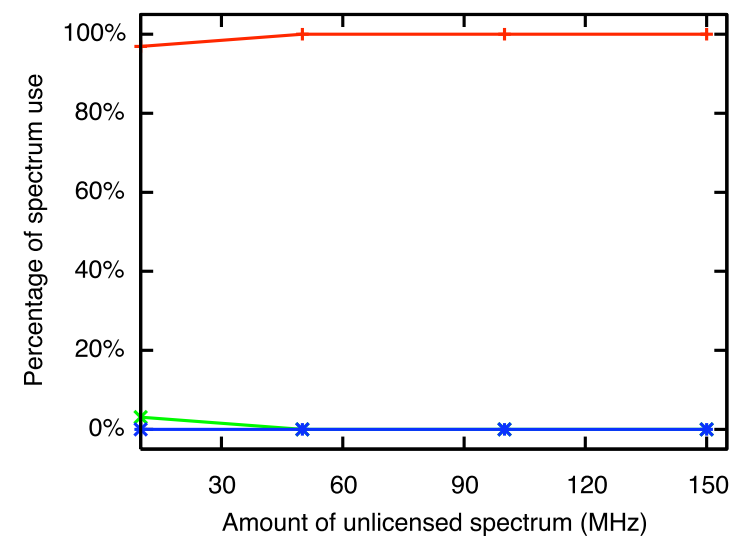

(b)

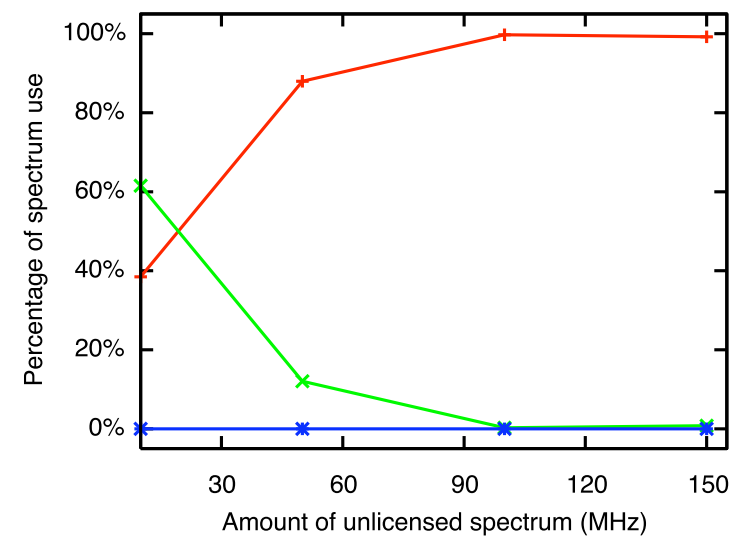

(d)

Figure 7.24: Percentage of spectrum access options vs. Amount of unlicensed spectrum for rigid application requirement: (a) $D_{\max }=10 \mathrm{~m}$, (b) $D_{\max }=100 \mathrm{~m}$, (c) $D_{\max }=250 \mathrm{~m}$, (d) $D_{\max }=500 \mathrm{~m}$, and (e) $D_{\max }=1000 \mathrm{~m}$. 
Table 7.11: Regression Analysis of Percentage Unlicensed Use on Amount of Unlicensed Spectrum and Characteristics of Secondary Users (Q6)

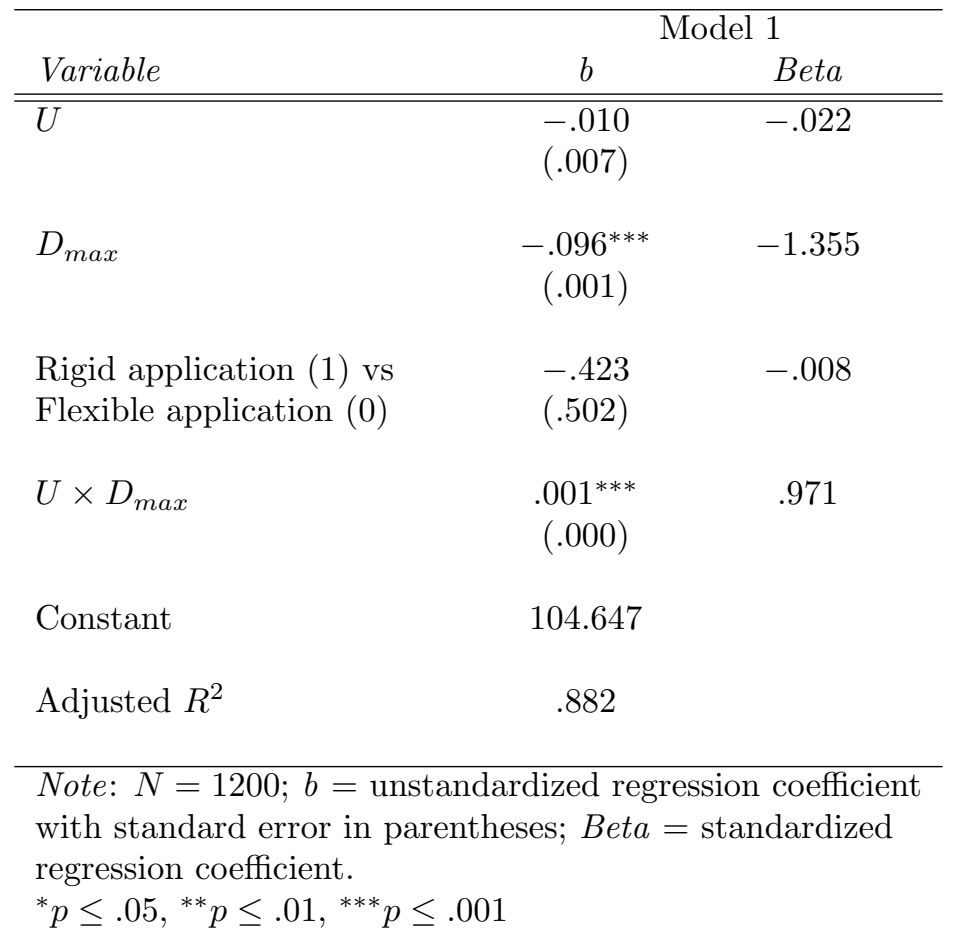


level of interference. Therefore, there is a substitution effect between the unlicensed use and the secondary use. As the coverage is increased to $500 \mathrm{~m}$ and $1000 \mathrm{~m}$ in Figures $7.24(\mathrm{~d})$ and (e), respectively, the substitution effect becomes highly noticeable.

We perform a statistical testing on Q6 with a multiple regression analysis shown in Table 7.11. The dependent variable in this case is the percentage of unlicensed spectrum use among consumer agents. The independent variables are $U, D_{\max }$, and the application requirements. The regression shows that $D_{\max }$ has a significant negative effect on the percentage of unlicensed use. It implies that an increase in $D_{\max }$ will result in a decrease in the unlicensed use. The interaction effect between $U$ and $D_{\max }$ also significantly influences the percentage of unlicensed use. As shown in Figures 7.24(a)-(e), the increase in $U$ must be combined with the large coverage scenarios for the increase in unlicensed use to become significant.

In summary, the results show that the rigid and flexible application requirements are not statistically different using the current specifications of the utility functions. Also, the expansion of unlicensed spectrum does not only benefit spectrum users with flexible application requirement and small coverage area, it is also useful for users with large coverage requirements. The results, however, demonstrate that, given a limited increase in the unlicensed band, those users with smaller coverage requirements can derive greater benefits by switching to the unlicensed use in a larger portion than spectrum users with large coverage requirements.

The results also suggest that the high-powered and large-coverage spectrum applications suffer greatly from the effect of the tragedy of the commons in the unlicensed band. In this case, the expansion of the unlicensed band alone cannot effectively accommodate this type of application. The benefits of the secondary use in this research question show that it is still essential to develop a flexible and market-based spectrum management approach. The additional allocation of spectrum for unlicensed use remains desirable especially for the low-powered and small-coverage applications. In this case, the risk of the tragedy of the commons can be reduced by the designs of etiquette for coexistence spectrum access $[41,42,43]$. Another technique is to implement a cognitive radio system, which can be used to achieve cooperative spectrum access [91]. Cognitive radio can be applied to both market- 
based and unlicensed spectrum sharing. However, this radio technology relies heavily on the capabilities of the wireless devices and the system architecture.

\subsection{IMPLICATIONS AND LIMITATIONS OF RESULTS}

As with other agent-based computational models, the limitations of results from the secondary use of spectrum model arise from the nature of the bottom-up modeling approach. Since the model requires a complete specification of initial parameters, behaviors, protocols, and institutional arrangement, the results are conditional upon the specific values of those parameters in the initial specifications. This section discusses two key issues that directly affect the experimental outcomes.

First, we assume that there is sufficient market thickness in the secondary use of radio spectrum. Raising the market thickness (i.e., the effective number of participants) increases the number of providers available to each consumer and the number of consumers available to each provider. In the analysis of research questions Q2-Q6 in Sections 7.4-7.8, the thickness needs to be sufficient in order to create secondary use activities and allow us to study the effects of other factors. The default numbers of consumers and providers in Table 7.1 are derived from the experiments on the number of participants (Q1) in Section 7.3. The default number of participants generate a moderate level of secondary use and are being used throughout Q2-Q6.

Second, the results are influenced by the initial specifications. For example, if the requirement of unlicensed spectrum access is changed from the frequency hopping scheme to CSMA $/ \mathrm{CA}^{5}$ protocol, the results of spectrum access options will also change. Any new results, however, should follow the same trend as demonstrated in this chapter. The goal of developing the agent-based model for secondary use is to provide the trend of spectrum access options in each scenario to assist policy makers in creating policy instruments rather than to give specific measurement in each of the special cases. The initial specifications of the model are derived from the fundamental concept of wireless communications and do

\footnotetext{
${ }^{5}$ Carrier Sense Multiple Access with Collision Avoidance.
} 
not rely on any specific technology. Still, the model is sufficiently flexible to replicate the real-world settings if the specific numerical results are needed. 


\subsection{CONCLUSION AND FUTURE WORK}

The necessity of spectrum reform as a result of ever-increasing demand for spectrum-based applications and services is evidenced by the outcomes of spectrum auctions. The technological advances in wireless devices create a possibility of moving towards more flexible and market-oriented approaches to spectrum management. In this dissertation, we focus on the development of secondary use of spectrum, defined as a temporary access of existing licensed spectrum by a user who does not own a license. We provide a comprehensive review of spectrum sharing approaches in Chapter 2, all of which aim to improve the efficiency of spectrum usage. The literature, however, shows that the studies on spectrum trading and leasing are very limited and mostly focus on policy and regulatory aspects. There are technical, economic, and strategic issues that must be considered all together for this new spectrum management approach to become feasible. Therefore, as a contribution, we provide a framework that combines issues necessary to be addressed in order to develop appropriate spectrum policies in Chapter 5.

One of the main concerns in secondary use of spectrum is the effects of transaction cost. It is generally agreed among researchers that transaction costs are the friction impeding market power and can prevent some desirable transactions from taking place. Instead of quantifying the amount of transaction costs directly, we propose the use of transaction cost economics (TCE) as a proxy. With TCE, we can define different forms of secondary use as well as incorporate the existing spectrum access methods (i.e., unlicensed use and exclusive license) into the model. The forms of secondary use may range from a spot spectrum market to a long-term lease or an MVNO, each of which can result in different magnitudes of transaction costs and levels of uncertainty due to interference between primary and secondary users. We also propose a common metric, degree of control, to differentiate and arrange organizational 
forms in which a spectrum transaction can take place. The degree of control allows us to speculate the trend of spectrum access methods from the outcome of each scenario.

As the main contribution of this dissertation, we propose a novel use of agent-based computational economics in conjunction with TCE to study the secondary use of radio spectrum in Chapter 6. The bottom-up approach of the agent-based spectrum access model allows an integration of economic and technical considerations to determine the pre-conditions of the secondary use concept. The agent-based modeling of spectrum users as consumer agents and provider agents, their learning processes, and the wireless environment in the spectrum access context are original. In addition to the specifications of agents, we also propose agent's opportunistic behavior in spectrum access transactions, which is one of the sources of transaction costs. Opportunism allows agents to take advantages in a trade and therefore could create interference to the transacting partners. We also implement a continuous double auction as a mechanism to determine the prices of spectrum access transactions between consumer and provider agents.

We use the agent-based model to conduct experiments in six research questions. The results in Chapter 7 show that the introduction of secondary use allows a portion of consumers who cannot afford an exclusive license or find it impractical to use to become secondary users. The following summarizes the findings of statistical testings.

- Secondary use of spectrum only emerges when there is a large number of participants. This condition creates a competitive market force to drive secondary use transactions and suppresses opportunistic behaviors of the secondary use participants.

- The density of wireless devices does not affect the choices of spectrum access. However, the path-loss exponent of the rural environment creates a higher secondary use than that of the urban environment.

- Secondary use is higher when primary users have continuous spectrum usage than intermittent spectrum usage. Synchronization between primary and secondary users appears to be a major factor in developing secondary use for primary users who exhibit an intermittent spectrum use.

- Spectrum users who require a large coverage area will move towards an exclusive license or hierarchy to organize spectrum access transactions because these organizational 
structures can provide greater guarantees against uncertainty and interference.

- Given the current specifications of rigid and flexible application requirements, the effect of application requirement and user's QoS sensitivity do not statistically influence the choices of spectrum access or the degree of control. However, the combination of a highlevel sensitivity and a large coverage requirement produces a significant effect that will move users towards an exclusive license or hierarchy to obtain spectrum access.

- The expansion of unlicensed spectrum is useful for both spectrum users with small and large coverage requirements. The users with smaller coverage, however, can derive greater benefits from an additional spectrum in the unlicensed band.

There is a great potential for future research. The agent-based model is a bottom-up layered design with fundamental elements in wireless communications such as transmitters and receivers. The association between these wireless devices forms a network configuration of spectrum users. Using an object-oriented technology, a new type of agents, behavioral method, and operating environment can be developed independently and easily integrated into the model. This modularity benefit allows the model to be customized for conducting experiments in various scenarios. The following discusses the possibility of extending the agent-based model for secondary use as well as studying other aspects of spectrum sharing.

- Secondary use of spectrum in the current model concerns with a temporary access of existing licensed spectrum owned by a primary user. The future model may include exclusive license trading in which a buyer would automatically become a primary user once s/he obtains an exclusive license.

- A new type of agent that represents a spectrum reseller or a speculator can be introduced into the model. A reseller would purchase spectrum with an intention of selling them for profit rather than using them. A speculator, on the other hand, has an intention of holding them and selling at some future time for profit. In the model, the spectrum users can be extended so that they can become spectrum resellers or speculators. In this case, they would buy excess amounts of spectrum and resell it, either on a temporary or permanent basis, to smaller users.

- As long as spectrum property right is not fully resolved, spectrum may not be allowed to 
trade freely without restrictions. The government may create an open-access spectrum band with minimal technical restrictions and license the spectrum to a private-sector band manager. The band manager needs to manage and monitor spectrum access to avoid interference within the band as well as to the outside. This spectrum management approach can be implemented as a band manager agent in the model. The commercial band manager will have a financial incentive in promoting both efficient use and technical innovation. Therefore, instead of using a double auction, the band manager may act as a central auctioneer who optimizes spectrum leasing in his/her own spectrum band.

- The experiments on the agent-based model are currently simplified by having homogeneous consumer agents and provider agents. A mixed population that reflects the real-world settings may produce interesting results. Each consumer and provider can be initialized with different parameter values including network configuration, coverage, spectrum requirement, utility function, and pricing parameters.

- In the agent-based model, the changes in parameters can reduce the amount of spectrum available for secondary use. However, most of the experiments are conducted with a large number of providers. Therefore, the reduction in spectrum supply only shows minimal effects. Conducting experiments with a smaller number of providers could generate a different set of results in which the effects of limited spectrum supply and potential opportunism could play a more important role.

- When provider agents utilize spectrum periodically, consumers may need to coordinate the timing with providers in order to efficiently operate as secondary users. The synchronization of spectrum access between consumers and providers can eliminate the constraint of access duration as mentioned in Section 7.5. In addition, the busy/idle duration of primary users and the access duration demanded by consumers should play an important role in secondary use, especially in the scenario where providers share variable amounts of spectrum. The future work can implement the synchronization mechanism and study the effects of spectrum access durations of both providers and consumers.

- In many cases, providers may not be able to continuously share a fixed amount of spectrum for secondary use. They may allocate a fixed portion for secondary use and reserve the rest for their own systems. This scenario creates a two-tier spectrum sharing for 
secondary use in which there is always a fixed amount available and the rest is subjected to provider's current spectrum usage. The two-tier spectrum sharing is not currently explored in this dissertation.

- The current spectrum access demand of consumers is a constant function. The future work can include an intermittent spectrum usage using periodic functions or aperiodic functions of spectrum demand into consumer agents, as in the current provider agents. In addition, two interesting parameters are not included in the current model: Money endowment and mobility. Spectrum users may be given an initial money endowment or a lifetime money endowment profile that represent a spectrum access budget. Using one of the mobility models in wireless communications also allows us to model mobile wireless devices in the secondary use environment. These two parameters are likely to produce another sets of results on the secondary use of radio spectrum.

- The transmitters and receivers in the model can be extended to study spectrum sharing in other dimensions such as time, geographical area, or spread spectrum. The potential subject in this area is the effects of advanced radio technologies, such as software-defined radio or cognitive radio, on the spectrum access methods. The reconfigurability capabilities of wireless devices and unconventional methods of spectrum access, such as ultra-wideband, are expected to favor the open spectrum policy, which calls for more spectrum for unlicensed access, rather than using market forces in the market-based spectrum management.

- It is also possible to apply the agent-model to other spectrum sharing techniques. In cooperative sharing schemes, interruptible spectrum sharing can be implemented by developing a new protocol and a new agent behavioral method. For example, a secondary user may be required to detect a positive signal from a primary user before s/he can transmit in the primary user's spectrum band. In coexistent sharing schemes, the agentbased model can be used to test different spectrum etiquette protocols for an unlicensed access. The effectiveness of each technique under consideration can be evaluated by inspecting the consumer's choice of spectrum access in the same way as this dissertation did for the secondary use. 


\section{BIBLIOGRAPHY}

[1] U.S. Code, Federal Communications Commission, "Industrial, scientific, and medical equipment," 47 CFR 18.107 (2004).

[2] The United Kingdom National Audit Office, "The auction of radio spectrum for the third generation of mobile phones," Reported by the Comptroller and Auditor General HC 233 Session 2001-2002, Oct. 2001.

[3] The United Kingdom Radiocommunications Agency. (2007, June) The UK third generation mobile auction. [Online]. Available: http://www.ofcom.org.uk/static/ archive/spectrumauctions/auction/auction_index.htm

[4] The German Federal Network Agency (Bundesnetzagentur). (2007, June) Universal Mobile Telecommunications System (UMTS). [Online]. Available: http://www. bundesnetzagentur.de

[5] (2007, June) UMTS and 3G contracts. [Online]. Available: http://www.umtsworld. com/industry/licenses.htm

[6] Federal Communications Commission, "Auction of advanced wireless services licenses closes; Winning bidders announced for auction no. 66," Public Notice: DA 06-1882, Sept. 2006.

[7] —_, "Spectrum policy task force report," ET Docket No. 02-135, Nov. 2002.

[8] —, "In the matter of promoting efficient use of spectrum through elimination of barriers to the development of secondary markets: Second report and order," FCC 04-167, WT Docket No. 00-230, Sept. 2004.

[9] A. Tonmukayakul and M. B. Weiss, "Secondary use of radio spectrum: A feasibility analysis," presented at the Telecommunications Policy Research Conference (TPRC'04), Arlington, VA, Oct. 1-3, 2004.

[10] M. B. Weiss, K. C. Huang, E. S. Myakotnykh, and A. Tonmukayakul, "Secondary use of electromagnetic spectrum: A survey of the issues," presented at the International Telecommunications Society (ITS'04), Berlin, Germany, Sept. 4-7, 2004. 
[11] A. Tonmukayakul and M. B. Weiss, "An agent-based model for secondary use of radio spectrum," in Proc. of IEEE International Symposium on Dynamic Spectrum Access Networks (IEEE DySPAN'05), Baltimore, MD, Nov. 8-11, 2005, pp. 467-475.

[12] —_, "A transaction cost analysis of secondary vs. unlicensed spectrum use," presented at the Telecommunications Policy Research Conference (TPRC'06), Arlington, VA, Sept. 29-Oct. 1, 2006.

[13] R. J. Matheson, "The electrospace model as a frequency management tool," in Proc. of the 5th Annual International Symposium on Advanced Radio Technologies (ISART'03), Boulder, CO, Mar. 4-7, 2003.

[14] L. H. Jones, "Operational spectrum effectiveness: A performance metric for software defined radio," in Proc. of IEEE Military Communications Conference (IEEE MILCOM'01), Vienna, VA, Oct. 2001.

[15] J. M. Peha, "Approaches to spectrum sharing," IEEE Commun. Mag., vol. 43, no. 2, pp. 10-12, Feb. 2005.

[16] G. R. Faulhaber and D. J. Faber, "Spectrum management: Property rights, markets, and the commons," AEI-Brookings Joint Center for Regulatory Studies, Working paper 02-12, Dec. 2002. [Online]. Available: http://www.aei-brookings.org/admin/ authorpdfs/page.php?id=217

[17] W. D. Horne, "Adaptive spectrum access: Using the full spectrum space," presented at the Telecommunications Policy Research Conference (TPRC'03), Arlington, VA, Sept. 2003.

[18] T. M. Valletti, "Spectrum trading," Telecommunications Policy, vol. 25, no. 10-11, pp. 655-670, Oct.-Nov. 2001.

[19] A. S. De Vany, "Implementing a market-based spectrum policy," Journal of Law and Economics, vol. 41, no. 2, pp. 627-646, Oct. 1998.

[20] R. H. Coase, "The federal communications commission," Journal of Law and Economics, vol. 2, pp. 1-40, Oct. 1959.

[21] A. S. De Vany, R. D. Eckert, C. J. Meyers, D. J. O'Hara, and R. C. Scott, "A property system for market allocation of the electromagnetic spectrum: A legal-economicengineering study," Stanford Law Review, vol. 21, no. 6, pp. 1499-1561, June 1969.

[22] R. J. Matheson, "Flexible spectrum use rights," in Proc. of the 7th Annual International Symposium on Advanced Radio Technologies (ISART'05), Boulder, CO, Mar. 1-3, 2005.

[23] D. N. Hatfield and P. J. Weiser, "Property rights in spectrum: Taking the next step," in Proc. of IEEE International Symposium on Dynamic Spectrum Access Networks (IEEE DySPAN'05), Baltimore, MD, Nov. 8-11, 2005, pp. 43-55. 
[24] J. Berresford and W. Leighton, "The law of property and the law of spectrum: A critical comparison," CommLaw Conspectus: Journal of Communications Law and Policy, vol. 13, no. 1, pp. 35-49, Mar. 2004.

[25] M. Falch and R. Tadayoni, "Economic versus technical approaches to frequency management," Telecommunications Policy, vol. 28, no. 2, pp. 197-211, Mar. 2004.

[26] M. M. Bykowsky, "A secondary market for the trading of spectrum: Promoting market liquidity," Telecommunications Policy, vol. 27, no. 7, pp. 533-541, Aug. 2003.

[27] J. M. Peha and S. Panichpapiboon, "Real-time secondary markets for spectrum," Telecommunications Policy, vol. 28, pp. 603-618, Aug.-Sept. 2004.

[28] R. Leese, P. Levine, and N. Rickman, "The economic effects of spectrum trading," in Proc. of Royal Economic Society Annual Conference, no. 123, Coventry, UK, Aug. 2002.

[29] J. Nolan, "Frequency assignment and spectrum trading for radio services," Ph.D. dissertation, University of Oxford, Oxford, UK, 2004.

[30] J. Marsh, "Secondary market in non-federal public safety spectrum," presented at the Telecommunications Policy Research Conference (TPRC'04), Arlington, VA, Oct. 1-3, 2004.

[31] M. M. Bykowsky and M. J. Marcus, "Facilitating spectrum management reform via callable/interruptible spectrum," presented at the Telecommunications Policy Research Conference (TPRC'02), Arlington, VA, Sept. 2002.

[32] Europe's Information Society. (2007, June) Dynamic Radio for IP-Services in Vehicular Environment (DRiVE). [Online]. Available: http://www.ist-drive.org

[33] — . (2007, June) Spectrum Efficient Uni- and Multicast Services over Dynamic Multi-Radio Networks in Vehicular Environment (OverDRiVE). [Online]. Available: http://www.comnets.rwth-aachen.de/ O_drive/index.html

[34] Motorola Labs. (2007, June) End-to-End Reconfigurability $\left(\mathrm{E}^{2} \mathrm{R}\right)$. [Online]. Available: http://e2r2.motlabs.com

[35] P. Demestichas and V. Stavroulaki, "Issues in introducing resource brokerage functionality in B3G composite radio environments," IEEE Wireless Commun. Mag., vol. 11, no. 5, pp. 32-40, Oct. 2004.

[36] P. Leaves, K. Moessner, R. Tafazolli, D. Grandblaise, D. Bourse, R. Tonjes, and M. Breveglieri, "Dynamic spectrum allocation in composite reconfigurable wireless networks," IEEE Commun. Mag., vol. 42, no. 5, pp. 72-81, May 2004.

[37] T. A. Weiss and F. K. Jondral, "Spectrum pooling: An innovative strategy for the enhancement of spectrum efficiency," IEEE Commun. Mag., vol. 42, no. 3, pp. S8-S14, Mar. 2004. 
[38] T. Kamakaris, M. M. Buddhikot, and R. Iyer, "A case for coordinated dynamic spectrum access in cellular networks," in Proc. of IEEE International Symposium on Dynamic Spectrum Access Networks (IEEE DySPAN'05), Baltimore, MD, Nov. 8-11, 2005, pp. 289-298.

[39] M. M. Buddhikot and K. Ryan, "Spectrum management in coordinated dynamic spectrum access based cellular networks," in Proc. of IEEE International Symposium on Dynamic Spectrum Access Networks (IEEE DySPAN'05), Baltimore, MD, Nov. 8-11, 2005, pp. 299-307.

[40] R. A. Linsenmayer, "Secondary spectrum markets in Europe," Master's thesis, Tufts University, Medford, MA, May 2001.

[41] D. P. Satapathy and J. M. Peha, "Spectrum sharing without licenses: Opportunities and dangers," in Interconnection and Internet: Selected papers from the 1996 Telecommunications Policy Research Conference, G. Rosston and D. Waterman, Eds. Mahwah, NJ: Lawrence Erlbaum Associates, 1997, pp. 49-75.

[42] — - "A novel co-existence algorithm for unlicensed variable power devices," in Proc. of IEEE International Conference on Communications (IEEE ICC'01), vol. 9, St. Petersburg, Russia, June 2001, pp. 2845-2849.

[43] W. Lehr and J. Crowcroft, "Managing shared access to a spectrum commons," in Proc. of IEEE International Symposium on Dynamic Spectrum Access Networks (IEEE DySPAN'05), Baltimore, MD, Nov. 8-11, 2005, pp. 420-444.

[44] J. Mitola, "Software radio architecture: A mathematical perspective," IEEE J. Select. Areas Commun., vol. 17, no. 4, pp. 514-538, Apr. 1999.

[45] S. Haykin, "Cognitive radio: Brain-empowered wireless communications," IEEE J. Select. Areas Commun., vol. 23, no. 2, pp. 201-220, Feb. 2005.

[46] J. Mitola and G. Q. Maguire, "Cognitive radio: Making software radios more personal," IEEE Personal Commun. Mag., vol. 6, no. 4, pp. 13-18, Aug. 1999.

[47] S. Mangold, Z. Zhong, K. Challapali, and C.-T. Chou, "Spectrum agile radio: Radio resource measurements for opportunistic spectrum usage," in Proc. of IEEE Global Telecommunications Conference (IEEE GLOBECOM'04), vol. 6, Dallas, TX, 2004, pp. 3467-3471.

[48] L. Berlemann, S. Mangold, and B. H. Walke, "Policy-based reasoning for spectrum sharing in cognitive radio networks," in Proc. of IEEE International Symposium on Dynamic Spectrum Access Networks (IEEE DySPAN'05), Baltimore, MD, Nov. 8-11, 2005, pp. 1-10.

[49] R. H. Coase, "The nature of the firm," Economica, New Series, vol. 4, no. 16, pp. 386-405, Nov. 1937. 
[50] O. E. Williamson, Markets and Hierarchies: Analysis and Antitrust Implications. New York: Free Press, 1975.

[51] — - "Transaction-cost economics: The governance of contractual relations," Journal of Law and Economics, vol. 22, no. 2, pp. 233-261, Oct. 1979.

[52] — - The Economic Institutions of Capitalism. New York: Free Press, 1985.

[53] — - "Comparative economic organization: The analysis of discrete structural alternatives," Administrative Science Quarterly, vol. 36, no. 2, pp. 269-296, June 1991.

[54] H. A. Shelanski and P. G. Klein, "Empirical research in transaction cost economics: A review and assessment," Journal of Law, Economics, ES Organization, vol. 11, no. 2, pp. 335-361, Oct. 1995.

[55] A. Rindfleisch and J. B. Heide, "Transaction cost analysis: Past, present, and future applications," Journal of Marketing, vol. 61, no. 4, pp. 30-54, Oct. 1997.

[56] R. J. David and S.-K. Han, "A systematic assessment of the empirical support for transaction cost economics," Strategic Management Journal, vol. 25, no. 1, pp. 39-58, Jan. 2004.

[57] M. D. Santoro and J. P. McGill, "The effect of uncertainty and asset co-specialization on governance in biotechnology alliances," Strategic Management Journal, vol. 26, no. 13, pp. 1261-1269, Dec. 2005.

[58] L. Tesfatsion, "Agent-based computational economics: A constructive approach to economic theory," in Handbook of Computational Economics, Volume 2: Agent-based Computational Economics, L. Tesfatsion and K. L. Judd, Eds. Amsterdam: North-Holland, 2006.

[59] T. C. Schelling, Micro Motives and Macro Behavior. New York: W. W. Norton, 1978.

[60] J. M. Epstein and R. Axtell, Growing artificial societies: Social science from the bottom up. Cambridge, MA: MIT Press, 1996.

[61] R. L. Axtell, J. M. Epstein, and H. P. Young, "The emergence classes in a multiagent bargaining model," in Social Dynamics, S. N. Durlauf and H. P. Young, Eds. Cambridge, MA: MIT Press, 2001.

[62] B. LeBaron, "Empirical regularities from interacting long- and short-memory investors in an agent-based stock market," IEEE Trans. Evol. Comput., vol. 5, no. 5, pp. 442-455, Oct. 2001.

[63] K. Izumi and K. Ueda, "Phase transition in a foreign exchange market-Analysis based on an artificial market approach," IEEE Trans. Evol. Comput., vol. 5, no. 5, pp. 456-470, Oct. 2001. 
[64] F. Luna and A. Perrone, Eds., Agent-based methods in economics and finance: Simulation in Swarm. Boston, MA: Kluwer Academic, 2002.

[65] D. W. Bunn and F. S. Oliveira, "Agent-based simulation-An application to the new electricity trading arrangements of England and Wales," IEEE Trans. Evol. Comput., vol. 5, no. 5, pp. 493-503, Oct. 2001.

[66] IEEE Transactions on Evolutionary Computation: Special issue on the agent-based modeling of evolutionary economic systems, vol. 5, no. 5, Oct. 2001.

[67] Journal of Economic Dynamics \& Control: Special issue on agent-based computational economics, vol. 25, no. 3-4, Mar. 2001.

[68] Computational Economics: Special issue on agent-based computational economics, vol. 18, no. 1, Aug. 2001.

[69] K. L. Judd, "Computationally intensive analyses in economics," in Handbook of Computational Economics, Volume 2: Agent-based Computational Economics, L. Tesfatsion and K. L. Judd, Eds. Amsterdam: North-Holland, 2006.

[70] M. Richiardi, "The promises and perils of agent-based computational economics," in Proc. of Italy's Assoc. of Cognitive Science: Second National Conference (AISC'04), Milan, Italy, 2004.

[71] M. J. North, N. T. Collier, and J. R. Vos, "Experiences creating three implementations of the Repast agent modeling toolkit," ACM Transactions on Modeling and Computer Simulation, vol. 16, no. 1, pp. 1-25, Jan. 2006.

[72] Repast Organization for Architecture and Development. (2007, June) Repast: Recursive Porous Agent Simulation Toolkit. [Online]. Available: http://repast.sourceforge.net

[73] S. Shenker, "Fundamental design issues for the future internet," IEEE J. Select. Areas Commun., vol. 13, no. 7, pp. 1176-1188, Sept. 1995.

[74] L. A. DaSilva, "Pricing for QoS-enabled networks: A survey," IEEE Commun. Surveys \&5 Tutorials, vol. 3, no. 2, pp. 2-8, Second Quarter 2000.

[75] X. Wang and H. Schulzrinne, "An integrated resource negotiation, pricing, and QoS adaptation framework for multimedia applications," IEEE J. Select. Areas Commun., vol. 18, no. 12, pp. 2514-2529, Dec. 2000.

[76] A. E. Roth and I. Erev, "Learning in extensive-form games: Experimental data and simple dynamic models in the intermediate term," Games and Economic Behavior, vol. 8, no. 1, pp. 164-212, Jan. 1995.

[77] I. Erev and A. E. Roth, "Predicting how people play games: Reinforcement learning in experimental games with unique, mixed strategy equilibria," The American Economic Review, vol. 88, no. 4, pp. 848-881, Sept. 1998. 
[78] R. S. Sutton and A. G. Barto, Reinforcement Learning: An Introduction. Cambridge, MA: MIT Press, 1998.

[79] T. B. Klos and B. Nooteboom, "Agent-based computational transaction cost economics," Journal of Economic Dynamics 83 Control, vol. 25, pp. 503-526, Mar. 2001.

[80] D. Friedman, "The double auction market institution: A survey," in The Double Auction Market: Institutions, Theories, and Evidence, D. Friedman and J. Rust, Eds. Reading, MA: Addison-Wesley, 1991, pp. 3-25.

[81] V. L. Smith, "An experimental study of competitive market behavior," Journal of Political Economy, vol. 70, no. 2, pp. 111-137, Apr. 1962.

[82] D. K. Gode and S. Sunder, "Allocative efficiency of markets with zero-intelligence traders: Market as a partial substitute for individual rationality," Journal of Political Economy, vol. 101, no. 1, pp. 119-137, Feb. 1993.

[83] D. Cliff and J. Bruten, "Minimal-intelligence agents for bargaining behaviors in marketbased environments," Hewlett Packard Laboratories, Bristol, UK, Technical Report HPL-97-91, Aug. 1997.

[84] P. R. Wurman, W. E. Walsh, and M. P. Wellman, "Flexible double auctions for electronic commerce: Theory and implementation," Decision Support Systems, vol. 24, no. 1, pp. 17-27, Nov. 1998.

[85] C. Preist and M. van Tol, "Adaptive agents in a persistent shout double auction," in Proc. of International Conference on Information and Computation Economies, Charleston, SC, 1998, pp. 11-18.

[86] C. Preist, "Commodity trading using an agent-based iterated double auction," in Proc. of International Conference on Autonomous Agents, Seattle, WA, 1999, pp. 131-138.

[87] D. E. Rumelhart, G. E. Hinton, and R. J. Williams, "Learning internal representations by error propagation," in Parallel Distributed Processing: Explorations in the Microstructure of Cognition, Vol. 1: Foundations, D. E. Rumelhart and J. L. McClelland, Eds. Cambridge, MA: MIT Press, 1986, pp. 318-362.

[88] W. Lehr, "Economic case for dedicated unlicensed spectrum below $3 \mathrm{GHz}$," New America Foundation, Washington, DC, Policy Papers, 2004.

[89] Y. Benkler, "Some economics of wireless communications," Harvard Journal of Law $\mathcal{E}^{3}$ Technology, vol. 16, no. 1, pp. 25-83, 2002.

[90] L. Lessig, The Future of Ideas: The Fate of the Commons in a Connected World. New York: Random House, 2001. 
[91] G. Ganesan and Y. Li, "Cooperative spectrum sensing in cognitive radio, Part II: Multiuser networks," IEEE Trans. Wireless Commun., vol. 6, no. 6, pp. 2214-2222, June 2007. 